\title{
Convergence of a linearly extrapolated BDF2 finite element scheme for viscoelastic fluid flow
}

Yunzhang Zhang ${ }^{1 *}$, Chao Xu$^{2}$ and Jiaquan Zhou ${ }^{2}$

\section{*Correspondence:}

z2004228@126.com

'School of Mathematics and

Statistics, Henan University of

Science and Technology, Luoyang, 471023, China

Full list of author information is

available at the end of the article

\begin{abstract}
The stability and convergence of a linearly extrapolated second order backward difference (BDF2-LE) time-stepping scheme for solving viscoelastic fluid flow in $\mathbb{R}^{d}$, $d=2,3$, are presented in this paper. The time discretization is based on the implicit scheme for the linear term and the two-step linearly extrapolated scheme for the nonlinear term. Mixed finite element (MFE) method is applied for the spatial discretization. The approximations of stress tensor $\sigma$, velocity vector $\mathbf{u}$ and pressure $p$ are $P_{m}$-discontinuous, $P_{k}$-continuous and $P_{q}$-continuous elements, respectively. Upwinding needed for convection of $\sigma$ is made by a discontinuous Galerkin (DG) FE method. For the time step $\Delta t$ small enough, the existence of an approximate solution is proven. If $m, k \geq \frac{d}{2}, q+1 \geq \frac{d}{2}$, and $\Delta t \leq C_{0} h^{\frac{d}{4}}$, then the discrete $H^{1}$ and $L^{2}$ errors for the velocity and stress, and $L^{2}$ error for the pressure, are bounded by $C\left(\Delta t^{2}+h^{\min \{m, k, q+1\}}\right)$, where $h$ denotes the mesh size. The derived theoretical results are supported by numerical tests.
\end{abstract}

MSC: 65N30; 65N12; 76A10

Keywords: viscoelastic fluid flow; linearly extrapolated BDF2; mixed finite element; discontinuous Galerkin; stability analysis; error estimate

\section{Introduction}

In this paper, we consider the time-dependent incompressible viscoelastic fluid flow problem

$$
\begin{aligned}
& \operatorname{Re}\left(\partial_{t} \mathbf{u}+\mathbf{u} \cdot \nabla \mathbf{u}\right)-\nabla \cdot \sigma-2(1-\alpha) \nabla \cdot D(\mathbf{u})+\nabla p=\mathbf{f}, \\
& \lambda\left(\partial_{t} \sigma+\mathbf{u} \cdot \nabla \sigma\right)+\sigma+\lambda g_{a}(\sigma, \nabla \mathbf{u})-2 \alpha D(\mathbf{u})=0, \\
& \nabla \cdot \mathbf{u}=0
\end{aligned}
$$

for $\mathbf{x} \in \Omega$ and $t \in(0, T]$, where $\Omega \subset \mathbb{R}^{d}(\mathrm{~d}=2,3)$ is a connected, bounded polygonal domain with the Lipschitz continuous boundary $\partial \Omega$. $p(\mathbf{x}, t)$ represents the pressure, $\mathbf{u}=$ $\left(u_{1}(\mathbf{x}, t), \ldots, u_{d}(\mathbf{x}, t)\right)$ the velocity vector, and $\sigma(\mathbf{x}, t)$ the stress tensor. $\sigma$ is the viscoelastic part of the total stress tensor $\sigma_{\text {tot }}=\sigma+2(1-\alpha) D(\mathbf{u})-p \mathbf{I}$. $\lambda$ is the Weissenberg number, $R e$ the Reynolds number, $\mathbf{f}(\mathbf{x}, t)$ the body forces acting on the fluid and $0<\alpha<1$

(c) The Author(s) 2017. This article is distributed under the terms of the Creative Commons Attribution 4.0 International License (http://creativecommons.org/licenses/by/4.0/), which permits unrestricted use, distribution, and reproduction in any medium, provided you give appropriate credit to the original author(s) and the source, provide a link to the Creative Commons license, and indicate if changes were made. 
may be considered as the fraction of viscoelastic viscosity. The gradient of $\mathbf{u}$ is defined as $(\nabla \mathbf{u})_{i, j}=\partial u_{i} / \partial x_{j} . D(\mathbf{u})=\frac{1}{2}\left(\nabla \mathbf{u}+\nabla \mathbf{u}^{T}\right)$ is the rate of the strain tensor. For all $a \in[-1,1]$, $g_{a}(\sigma, \nabla \mathbf{u})$ is defined by

$$
g_{a}(\sigma, \nabla \mathbf{u})=\frac{1-a}{2}\left(\sigma \nabla \mathbf{u}+(\nabla \mathbf{u})^{T} \sigma\right)-\frac{1+a}{2}\left((\nabla \mathbf{u}) \sigma+\sigma(\nabla \mathbf{u})^{T}\right) .
$$

The boundary and initial conditions are given by

$$
\begin{aligned}
& \mathbf{u}(\mathbf{x}, t)=\mathbf{0}, \quad \text { on } \partial \Omega \times(0, T], \\
& \mathbf{u}(\mathbf{x}, 0)=\mathbf{u}_{0}(\mathbf{x}), \quad \sigma(\mathbf{x}, 0)=\sigma_{0}(\mathbf{x}), \quad \forall \mathbf{x} \in \Omega .
\end{aligned}
$$

Time-dependent calculations of viscoelastic fluid flows are important to the understanding of many problems in non-Newtonian fluid mechanics, particularity those related to flow instabilities [1-3]. The existence and uniqueness of solutions to viscoelastic fluid flow (1.1a)-(1.4) were discussed in $[4,5]$.

Numerical methods for solving the time-dependent incompressible viscoelastic fluid flow have been investigated extensively [6-18]. For the analysis of the time-dependent problem, Baranger and Wardi [11] studied a DG approximation to inertialess flow in $\mathbb{R}^{2}$. Assuming the Hood-Taylor FE pair approximation for the velocity and pressure, and a discontinuous linear FE approximation for the stress, and Euler implicit method in time, under the assumption $\triangle t \leq C h^{3 / 2}$, they obtained that the discrete $H^{1}$ and $L^{2}$ errors for the velocity and stress, respectively, are bounded by $C\left(\Delta t+h^{3 / 2}\right)$. Ervin and Heuer [14] analyzed a fully discrete approximation for the time-dependent viscoelasticity equations with an Oldroyd B constitutive equation in $\mathbb{R}^{d}, d=2,3$. They used a Crank-Nicolson discretization for the time derivatives. At each time level a linear system of equations is solved. To resolve the nonlinear terms, they used a three-step extrapolation for the prediction of the velocity and stress at the new time level. The approximation is stabilized by using a discontinuous Galerkin approximation for the constitutive equation. Assume that $\Delta t$ is sufficiently small and satisfying $\Delta t \leq C h^{d / 4}$, the existence of an approximate solution is proven. A priori error estimate for the approximation in terms of $\Delta t$ and $h$ is also derived. In [12], Ervin and Miles analyzed the finite element spacial semi-discrete and Euler semi-implicit fully discrete schemes, which were stabilized by using a streamline upwind Petrov-Galerkin (SUPG) for the constitutive equation. Bensaada and Esselaoui in [15] presented error analysis of a modified Euler-SUPG approximation for the time-dependent viscoelastic flow problem. In [18], based on a splitting of the error into two parts: the error from the time discretization of the PDEs and the error from the finite element approximation of corresponding iterated time-discrete PDEs, the authors carried on unconditional error estimates for time-dependent viscoelastic fluid flow.

In this work, we consider the convergence of BDF2-LE in time and MFE in space for the viscoelastic fluid flow. The backward difference formula (BDF) class of multi-step schemes has been widely used as time integration method for both ordinary and partial differential equations, see [19-27]. The BDF2 is one of the most popular BDF schemes due to its stability and damping properties [28]. Girault and Raviart introduced and analyzed a firstorder and second-order BDF temporal semi-discrete schemes for Navier-Stokes equations in [21]. An unconditionally stable decoupled BDF2 time-stepping scheme was analyzed for 
Boussinesq type Navier-Stokes equation in [24]. To the best of our knowledge, there is no rigorous convergence analysis available yet for the viscoelastic fluid flow by using BDF2-LE in time. We will propose and analyze a coupled scheme which belongs to this class.

This article is organized as follows. In the next section, we introduce some notations and preliminaries related to a continuum and discrete problem. In Section 3, we propose the extrapolated time-stepping scheme, prove the existence of the numerical solution and establish the stability analysis. The error analysis for the general scheme is presented in Section 4. We also present numerical tests to confirm the theoretical results in Section 5. Finally, some conclusions are drawn.

\section{Notation and preliminaries}

We denote the $L^{2}(\Omega)$ norms and corresponding inner products by $\|\cdot\|$ and $(\cdot, \cdot)$. Likewise, the $L^{2}(\Omega)$ norms and the Sobolev $W^{k, p}(\Omega)$ norms [29] are denoted by $\|\cdot\|_{L^{p}}$ and $\|\cdot\|_{W^{k, p}}$, respectively. $H^{k}(\Omega)$ is used to represent the Sobolev space $W^{k, 2}(\Omega)$ and $\|\cdot\|_{k}$ denotes the norm in $H^{k}(\Omega)$. The space $H^{-k}(\Omega)$ denotes the dual spaces of $H_{0}^{k}(\Omega)$. All other norms will be clearly labeled with subscripts.

The velocity and pressure spaces are $X=H_{0}^{1}(\Omega)^{d}, Q=L_{0}^{2}(\Omega)$, respectively. The stress space $S$ and divergence-free functions space $V$ are given by

$$
\begin{aligned}
S= & \left\{\tau=\left(\tau_{i j}\right) ; \tau_{i j}=\tau_{j i} ; \tau_{i j} \in L^{2}(\Omega) ; 1 \leq i, j \leq d\right\} \\
& \cap\left\{\tau=\left(\tau_{i j}\right) ; \mathbf{v} \cdot \nabla \tau \in L^{2}(\Omega)^{d \times d}, \forall \mathbf{v} \in X\right\}, \\
V= & \{\mathbf{v} \in X ;(q, \nabla \cdot \mathbf{v})=0, \forall q \in Q\} .
\end{aligned}
$$

A weak formulation of (1.1a)-(1.1c) is as follows: Find $(\sigma, \mathbf{u}, p):[0, T] \rightarrow(S, X, Q)$ for a.e. $t \in(0, T]$ satisfying

$$
\begin{aligned}
& \operatorname{Re}\left(\partial_{t} \mathbf{u}, \mathbf{v}\right)+\operatorname{Rec}(\mathbf{u}, \mathbf{u}, \mathbf{v})+(\sigma, D(\mathbf{v}))+2(1-\alpha)(D(\mathbf{u}), D(\mathbf{v})) \\
& \quad-(p, \nabla \cdot \mathbf{v})=(\mathbf{f}, \mathbf{v}), \\
& (q, \nabla \cdot \mathbf{u})=0, \\
& \lambda\left(\partial_{t} \sigma+\mathbf{u} \cdot \nabla \sigma, \tau\right)+\lambda\left(g_{a}(\sigma, \nabla \mathbf{u}), \tau\right)+(\sigma, \tau)-2 \alpha(D(\mathbf{u}), \tau)=\mathbf{0}
\end{aligned}
$$

for all $(\tau, \mathbf{v}, q) \in(S, X, Q)$ with the initial condition (1.4) a.e. in $\Omega$, where the trilinear operator $c$ on $X \times X \times X$ is

$$
c(\mathbf{u}, \mathbf{v}, \mathbf{w})=(\mathbf{u} \cdot \nabla \mathbf{v}, \mathbf{w}) .
$$

By virtue of the divergence-free space $V$, the weak formulation of (1.1a)-(1.1c) can be written as follows: Find $(\sigma, \mathbf{u}) \in(S, V)$ such that, for all $(\tau, \mathbf{v}) \in(S, V)$,

$$
\begin{aligned}
& \operatorname{Re}\left(\partial_{t} \mathbf{u}, \mathbf{v}\right)+\operatorname{Rec}(\mathbf{u}, \mathbf{u}, \mathbf{v})+(\sigma, D(\mathbf{v}))+2(1-\alpha)(D(\mathbf{u}), D(\mathbf{v})) \\
& \quad=(\mathbf{f}, \mathbf{v}), \\
& \lambda\left(\partial_{t} \sigma+\mathbf{u} \cdot \nabla \sigma, \tau\right)+(\sigma, \tau)+\lambda\left(g_{a}(\sigma, \nabla \mathbf{u}), \tau\right)-2 \alpha(D(\mathbf{u}), \tau)=\mathbf{0} .
\end{aligned}
$$


Here we assume that the initial-boundary value problem (1.1a)-(1.4) has a unique solution satisfying the regularity conditions

$$
\begin{aligned}
& \mathbf{u} \in L^{2}\left(0, T ; H^{k+1}(\Omega)^{d}\right), \quad \partial_{t} \mathbf{u} \in L^{2}\left(0, T ; H^{k+1}(\Omega)^{d}\right), \quad \partial_{t}^{3} \mathbf{u} \in L^{2}\left(0, T ; L^{2}(\Omega)^{d}\right), \\
& p \in L^{2}\left(0, T ; H^{q+1}(\Omega)\right), \quad \sigma \in L^{2}\left(0, T ; H^{m+1}(\Omega)^{d \times d}\right), \\
& \partial_{t} \sigma \in L^{2}\left(0, T ; H^{m+1}(\Omega)^{d \times d}\right), \quad \partial_{t}^{3} \sigma \in L^{2}\left(0, T ; L^{2}(\Omega)^{d \times d}\right), \\
& \|\mathbf{u}\|_{\infty},\|\sigma\|_{\infty},\|\nabla \mathbf{u}\|_{\infty},\|\nabla \sigma\|_{\infty} \leq M \quad \text { for all } t \in[0, T] .
\end{aligned}
$$

By using $\nabla \cdot \mathbf{u}=0$ and $\mathbf{u}=\mathbf{0}$ on $\partial \Omega$, it is easy to see that $2(D(\mathbf{u}), D(\mathbf{v}))=(\nabla \mathbf{u}, \nabla \mathbf{v})$, and $\|D(\mathbf{u})\| \leq\|\nabla \mathbf{u}\|$.

In order to keep the exposition simple, we restrict our attention to convex polyhedral domains. Suppose that $T^{h}$ is a uniformly regular triangulation of $\Omega$ such that $\Omega=\{\bigcup K$ : $\left.K \in T^{h}\right\}$ and assume that there exist positive constants $v_{1}, v_{2}$ such that $v_{1} h \leq h_{K} \leq v_{2} \rho_{K}$, where $h_{K}$ is the diameter of $K, \rho_{K}$ is the diameter of the greatest ball included in $K$, and $h=\max _{K \in T^{h}} h_{K}$. The corresponding FE spaces are

$$
\begin{aligned}
& X_{h}=\left\{\mathbf{v} \in X \cap C^{0}(\bar{\Omega})^{d} ; \mathbf{v}_{\mid K} \in P_{k}(K)^{d}, \forall K \in T^{h}\right\}, \\
& S_{h}=\left\{\tau \in S ; \tau_{\mid K} \in P_{m}(K)^{d \times d} ; \forall K \in T^{h}\right\}, \\
& Q_{h}=\left\{q \in Q \cap C^{0}(\bar{\Omega}) ; q_{\mid K} \in P_{q}(K) ; \forall K \in T^{h}\right\},
\end{aligned}
$$

where $P_{m}(K)$ denotes the space of polynomials of degree $\leq m$ on $K \in T^{h}$.

We make the following assumptions on the finite dimensional subspaces.

Assumption A1 For $(\mathbf{u}, p) \in H^{k+1}(\Omega)^{d} \times H^{q+1}(\Omega)$, there exists $\left(\Pi_{\mathbf{u}}(\mathbf{u}), \Pi_{p}(p)\right) \in V_{h} \times Q_{h}$ such that $[21,30-33]$

$$
\begin{aligned}
& \left\|\mathbf{u}-\Pi_{\mathbf{u}}(\mathbf{u})\right\|+h\left\|\nabla\left(\mathbf{u}-\Pi_{\mathbf{u}}(\mathbf{u})\right)\right\| \leq C_{i p} h^{k+1}\|\mathbf{u}\|_{k+1}, \\
& \left\|p-\Pi_{p}(p)\right\| \leq C_{i p} h^{q+1}\|p\|_{q+1} .
\end{aligned}
$$

Let $\Pi_{\sigma}(\sigma) \in S_{h}$ be a $P_{m}$ continuous interpolant of $\sigma$, and if $\sigma \in H^{m+1}(\Omega)^{d \times d}$, we have that

$$
\left\|\sigma-\Pi_{\sigma}(\sigma)\right\|+h\left\|\nabla\left(\sigma-\Pi_{\sigma}(\sigma)\right)\right\| \leq C_{i p} h^{m+1}\|\sigma\|_{m+1} .
$$

Assumption A2 (Discrete inf-sup condition) For each $q_{h} \in Q_{h}$, there exists a nonzero function $\mathbf{v}_{h} \in X_{h}$ such that

$$
\inf _{q_{h} \in Q_{h}} \sup _{\mathbf{v}_{h} \in X_{h}} \frac{\left(q_{h}, \nabla \cdot \mathbf{v}_{h}\right)}{\left\|q_{h}\right\|\left\|\nabla \mathbf{v}_{h}\right\|} \geq \beta>0
$$

where $\beta$ is a positive constant independent of the mesh size $h$. 
Assumption A3 For each $\omega_{h} \in X_{h}$, one has the inverse inequality, the Poincare inequality and the second Korn's inequality

$$
\begin{aligned}
& \left\|\nabla \omega_{h}\right\| \leq C_{i} h^{-1}\left\|\omega_{h}\right\|, \quad\left\|\omega_{h}\right\|_{\infty} \leq C_{i} h^{-\frac{d}{2}}\left\|\omega_{h}\right\|, \\
& \left\|\omega_{h}\right\| \leq C_{p}\left\|\nabla \omega_{h}\right\|, \\
& \left\|\nabla \omega_{h}\right\| \leq C_{k}\left\|D\left(\omega_{h}\right)\right\|,
\end{aligned}
$$

where $C_{i}, C_{p}$ and $C_{k}$ are the positive constants, which only depend on $\Omega$.

There are many finite element spaces satisfying Assumptions A1-A3, such as the MINI $\left(P_{1} b, P_{1}\right)$ elements, or the Hood-Taylor $\left(P_{2}, P_{1}\right)$ elements for the velocity $\mathbf{u}$ and pressure $p$, and $P_{1}$ (or $P_{2}$ ) discontinuous element for stress tensor $\sigma$.

The discretely divergence-free velocity space is denoted by

$$
V_{h}=\left\{\mathbf{v} \in X_{h} ;(q, \nabla \cdot \mathbf{v})=0 \text {, for all } q \in Q_{h}\right\} .
$$

Remark 2.1 The divergence-free space $V_{h}$ is introduced only for theoretical analysis. The practical computation should be based on the finite element space pair $\left(X_{h}, Q_{h}\right)$ for velocity and pressure. We refer the readers to Heywood and Rannacher [34, 35] for the details on the construction of $\left(X_{h}, Q_{h}\right)$.

Here we present a result which will be used in the stability analysis and error estimate for pressure. Since the divergence-free space $V_{h} \subset X_{h}$, we can define the norms of the dual spaces $X_{h}, V_{h}$ by

$$
\|\omega\|_{X_{h}^{\prime}}=\sup _{\mathbf{v}_{h} \in X_{h}} \frac{\left(\omega, \mathbf{v}_{h}\right)}{\left\|\nabla \mathbf{v}_{h}\right\|}, \quad\|\omega\|_{V_{h}^{\prime}}=\sup _{\mathbf{v}_{h} \in V_{h}} \frac{\left(\omega, \mathbf{v}_{h}\right)}{\left\|\nabla \mathbf{v}_{h}\right\|} .
$$

Lemma $2.2([36-38])$ For $\forall \mathbf{v} \in V_{h}$, the norms $\|\mathbf{v}\|_{X_{h}^{\prime}}$ and $\|\mathbf{v}\|_{V_{h}^{\prime}}$ are equivalent.

In order to describe the approximation of the constitutive equation by the method of discontinuous finite elements, following [6], we define

$$
\partial K^{-}(\mathbf{u})=\{\mathbf{x} \in \partial K ; \mathbf{u}(\mathbf{x}) \cdot \mathbf{n}(\mathbf{x})<0\}
$$

where $\partial K$ is the boundary of $K \in T^{h}$, and $\mathbf{n}$ is the outward unit normal to $\partial K$, and

$$
\partial \Omega^{h}=\left\{\bigcup \partial K: K \in T^{h}\right\} \backslash \partial \Omega, \quad \tau^{ \pm}(\mathbf{u})(\mathbf{x})=\lim _{\varepsilon \rightarrow 0^{ \pm}} \tau(\mathbf{x}+\varepsilon \mathbf{u}(\mathbf{x})) .
$$

Also, for all functions in $\prod_{K \in T_{h}}\left[H^{1}(K)\right]^{d \times d}$, we define

$$
\begin{aligned}
& (\sigma, \tau)_{h}=\sum_{K \in T_{h}}(\sigma, \tau)_{K}, \\
& \left\langle\sigma^{ \pm}, \tau^{ \pm}\right\rangle_{h, u}=\sum_{K \in T_{h}} \int_{\partial K^{-}(u)}\left(\sigma^{ \pm}(\mathbf{u}), \tau^{ \pm}(\mathbf{u})\right)|\mathbf{n} \cdot \mathbf{u}| d s, \\
& \left\langle\sigma^{ \pm}\left\|_{h, u}^{2}=\left\langle\sigma^{ \pm}, \sigma^{ \pm}\right\rangle_{h, u^{\prime}}, \quad\right\| \tau \|_{0, \Gamma^{h}}=\left(\sum_{K \in T_{h}}|\tau|_{0, \partial K}^{2}\right)^{1 / 2} .\right.
\end{aligned}
$$


The convection term $((\mathbf{u} \cdot \nabla) \sigma, \tau)$ is approximated by means of an operator $B$ on $X_{h} \times$ $S_{h} \times S_{h}$, defined by

$$
\begin{aligned}
& B(\mathbf{u}, \sigma, \tau) \\
& \quad=((\mathbf{u} \cdot \nabla) \sigma, \tau)_{h}+\frac{1}{2}(\nabla \cdot \mathbf{u} \sigma, \tau)+\left\langle\sigma^{+}-\sigma^{-}, \tau^{+}\right\rangle_{h, \mathbf{u}} \\
& \quad=-((\mathbf{u} \cdot \nabla) \tau, \sigma)_{h}-\frac{1}{2}(\nabla \cdot \mathbf{u} \tau, \sigma)+\left\langle\sigma^{-}, \tau^{-}-\tau^{+}\right\rangle_{h, \mathbf{u}^{\prime}}
\end{aligned}
$$

which implies some 'coercivity' of B [39]:

$$
B(\mathbf{u}, \sigma, \sigma)=\frac{1}{2}\left\|\sigma^{+}-\sigma^{-}\right\|_{h, \mathbf{u}}^{2} .
$$

Let $\left\{t_{n} \mid t_{n}=n \Delta t ; 0 \leq n \leq N\right\}$ be a uniform partition of [0,T] with the time step $\Delta t=$ $T / N$. We denote $\omega^{m}=\omega\left(\mathbf{x}, t_{m}\right)$. For a sequence of functions $\left\{\omega^{n}\right\}_{n=0}^{N}$, we define the BDF2 operator $\beth\left(\omega^{n+1}\right)$ and the linearly extrapolated operator $\digamma\left(\omega^{n+1}\right)$

$$
\beth\left(\omega^{n+1}\right)=\frac{3 \omega^{n+1}-4 \omega^{n}+\omega^{n-1}}{2 \triangle t}, \quad \digamma\left(\omega^{n+1}\right)=2 \omega^{n}-\omega^{n-1} .
$$

It follows from Taylor's formula with integral remainder that [26]

$$
\begin{aligned}
& \beth\left(\omega\left(t_{n}\right)\right)=\partial_{t} \omega\left(t_{n}\right)+\frac{1}{2 \triangle t} \int_{t_{n-2}}^{t_{n}}\left\{2\left(t-t_{n-1}\right)_{+}^{2}-\frac{1}{2}\left(t-t_{n-2}\right)^{2}\right\} \partial_{t}^{3} \omega d t, \\
& \digamma\left(\omega\left(t_{n}\right)\right)=\omega\left(t_{n}\right)+\int_{t_{n-2}}^{t_{n}}\left\{2\left(t-t_{n-1}\right)_{+}-\left(t-t_{n-2}\right)\right\} \partial_{t}^{2} \omega d t,
\end{aligned}
$$

where $\left(t-t_{n-1}\right)_{+}=\max \left(\left(t-t_{n-1}\right), 0\right)$. By the Cauchy-Schwarz inequality, we have the truncation error

$$
\begin{gathered}
\left\|\beth\left(\omega\left(t_{n}\right)\right)-\partial_{t} \omega\left(t_{n}\right)\right\| \leq C_{T}(\Delta t)^{3 / 2}\left\|\partial_{t}^{3} \omega(t)\right\|_{L^{2}\left(t_{n-2}, t_{n} ; L^{2}(\Omega)\right)}, \\
\left\|\digamma\left(\omega\left(t_{n}\right)\right)-\omega\left(t_{n}\right)\right\| \leq C_{T}(\Delta t)^{3 / 2}\left\|\partial_{t}^{2} \omega(t)\right\|_{L^{2}\left(t_{n-2}, t_{n} ; L^{2}(\Omega)\right)}
\end{gathered}
$$

where the constant $C_{T}$ is derived from Taylor's formula.

The BDF2 operator $\beth\left(\omega\left(t_{n+1}\right)\right)$ satisfies the relation [26]

$$
\begin{aligned}
& \left(\beth\left(\omega^{n+1}\right), \omega^{n+1}\right) \\
& =\frac{3}{4 \triangle t}\left\|\omega^{n+1}\right\|^{2}+\frac{1}{4 \triangle t}\left\|\omega^{n+1}-2 \omega^{n}+\omega^{n-1}\right\|^{2}-\frac{1}{\Delta t}\left\|\omega^{n}\right\|^{2} \\
& \quad+\frac{1}{2 \triangle t}\left[\left\|\omega^{n+1}-\omega^{n}\right\|^{2}-\left\|\omega^{n}-\omega^{n-1}\right\|^{2}\right]+\frac{1}{4 \triangle t}\left\|\omega^{n-1}\right\|^{2} .
\end{aligned}
$$

The discrete Gronwall's lemma [35] plays an important role in the following analysis.

Lemma 2.3 Let $\triangle t, H$, and $a_{n}, b_{n}, c_{n}, \gamma_{n}$ (for integers $n \geq 0$ ) be nonnegative numbers such that

$$
a_{l}+\Delta t \sum_{n=0}^{l} b_{n} \leq \Delta t \sum_{n=0}^{l} \gamma_{n} a_{n}+\Delta t \sum_{n=0}^{l} c_{n}+H \quad \text { for } l \geq 0 .
$$


Suppose that $\Delta t \gamma_{n}<1$ for all $n$, and set $\zeta_{n}=\left(1-\Delta t \gamma_{n}\right)^{-1}$. Then

$$
a_{l}+\Delta t \sum_{n=0}^{l} b_{n} \leq \exp \left(\Delta t \sum_{n=0}^{l} \gamma_{n} \zeta_{n}\right)\left(\Delta t \sum_{n=0}^{l} c_{n}+H\right) \text { for } l \geq 0
$$

Remark 2.4 If the first sum on the right in (2.14) extends only up to $l-1$, then estimate (2.15) holds for all $n>0$ with $\zeta_{n}=1$.

Throughout the paper, the constants $C_{1}, C_{2}, \ldots$ denote different constants which are independent of $h$ and $\triangle t$.

\section{Numerical scheme and its stability}

In this section, we first present the linearly extrapolated BDF2 scheme, then study the existence of numerical solutions, and finally establish stability of the numerical scheme.

\subsection{Numerical scheme}

Scheme 3.1 (BDF2-LE Galerkin FEM) Given $\mathbf{u}_{h}^{-1}=\mathbf{u}_{h}^{0}=\Pi_{\mathbf{u}}\left(\mathbf{u}_{0}\right) \in V_{h}, \sigma_{h}^{-1}=\sigma_{h}^{0}=$ $\Pi_{\sigma}\left(\sigma_{0}\right) \in S_{h}$, find $\mathbf{u}_{h}^{n+1} \in X_{h}, p_{h}^{n+1} \in Q_{h}, \sigma_{h}^{n+1} \in S_{h}$ for $n=0,1,2, \ldots, N-1$ satisfying

$$
\begin{aligned}
& \operatorname{Re}\left(\beth\left(\mathbf{u}_{h}^{n+1}\right), \mathbf{v}_{h}\right)+\operatorname{Rec}\left(\digamma\left(\mathbf{u}_{h}^{n+1}\right), \mathbf{u}_{h}^{n+1}, \mathbf{v}_{h}\right)+\left(\sigma_{h}^{n+1}, D\left(\mathbf{v}_{h}\right)\right) \\
& \quad+2(1-\alpha)\left(D\left(\mathbf{u}_{h}^{n+1}\right), D\left(\mathbf{v}_{h}\right)\right)-\left(p_{h}^{n+1}, \nabla \cdot \mathbf{v}_{h}\right)=\left(\mathbf{f}^{n+1}, \mathbf{v}_{h}\right), \\
& \left(q_{h}, \nabla \cdot \mathbf{u}_{h}^{n+1}\right)=0, \\
& \lambda\left(\beth\left(\sigma_{h}^{n+1}\right), \tau_{h}\right)+\left(\sigma_{h}^{n+1}, \tau_{h}\right)+\lambda B\left(\digamma\left(\mathbf{u}_{h}^{n+1}\right), \sigma_{h}^{n+1}, \tau_{h}\right) \\
& \quad-2 \alpha\left(D\left(\mathbf{u}_{h}^{n+1}\right), \tau_{h}\right)+\lambda\left(g_{a}\left(\digamma\left(\sigma_{h}^{n+1}\right), \nabla \mathbf{u}_{h}^{n+1}\right), \tau_{h}\right)=0
\end{aligned}
$$

for all $\mathbf{v}_{h} \in X_{h}, q_{h} \in Q_{h}$ and $\tau_{h} \in S_{h}$.

By virtue of the divergence-free subspace $V_{h}$, Scheme 3.1 can be written as another form (Scheme 3.2) which is used in stability analysis in this section and error analysis in Section 4.

Scheme 3.2 (BDF2-LE Galerkin FEM) Given $\mathbf{u}_{h}^{-1}=\mathbf{u}_{h}^{0}=\Pi_{\mathbf{u}}\left(\mathbf{u}_{0}\right) \in V_{h}, \sigma_{h}^{-1}=\sigma_{h}^{0}=$ $\Pi_{\sigma}\left(\sigma_{0}\right) \in S_{h}$, find $\mathbf{u}_{h}^{n+1} \in V_{h}, \sigma_{h}^{n+1} \in S_{h}$ for $n=0,1,2, \ldots, N-1$, satisfying

$$
\begin{aligned}
& \operatorname{Re}\left(\beth\left(\mathbf{u}_{h}^{n+1}\right), \mathbf{v}_{h}\right)+\operatorname{Rec}\left(\digamma\left(\mathbf{u}_{h}^{n+1}\right), \mathbf{u}_{h}^{n+1}, \mathbf{v}_{h}\right)+\left(\sigma_{h}^{n+1}, D\left(\mathbf{v}_{h}\right)\right) \\
& \quad+2(1-\alpha)\left(D\left(\mathbf{u}_{h}^{n+1}\right), D\left(\mathbf{v}_{h}\right)\right)=\left(\mathbf{f}^{n+1}, \mathbf{v}_{h}\right), \\
& \lambda\left(\beth\left(\sigma_{h}^{n+1}\right), \tau_{h}\right)+\left(\sigma_{h}^{n+1}, \tau_{h}\right)+\lambda B\left(\digamma\left(\mathbf{u}_{h}^{n+1}\right), \sigma_{h}^{n+1}, \tau_{h}\right) \\
& \quad-2 \alpha\left(D\left(\mathbf{u}_{h}^{n+1}\right), \tau_{h}\right)+\lambda\left(g_{a}\left(\digamma\left(\sigma_{h}^{n+1}\right), \nabla \mathbf{u}_{h}^{n+1}\right), \tau_{h}\right)=0
\end{aligned}
$$

for all $\mathbf{v}_{h} \in V_{h}$ and $\tau_{h} \in S_{h}$.

We see that Scheme 3.1 (or Scheme 3.2) is a linear extrapolation (semi-implicit) scheme, which is preferred over a fully implicit scheme (see Scheme 5.1 in Section 5) as it requires only solving the linear system in each time level. 
Remark 3.3 Since Scheme 3.1 is a two-step scheme, it requires starting values $\left(\mathbf{u}_{h}^{0}, \sigma_{h}^{0}\right)$ and $\left(\mathbf{u}_{h}^{1}, \sigma_{h}^{1}\right)$ and both with second order accuracy. For simplicity, here we take $\left(\mathbf{u}_{h}^{-1}, \sigma_{h}^{-1}\right)=$ $\left(\mathbf{u}_{h}^{0}, \sigma_{h}^{0}\right)=\left(\Pi_{\mathbf{u}}\left(\mathbf{u}_{0}\right), \Pi_{\sigma}\left(\sigma_{0}\right)\right)$, it ensures that $\left(\mathbf{u}_{h}^{1}, \sigma_{h}^{1}\right)$ is second order accuracy. We can also use the way as $[20,24]$ to get the value $\left(\mathbf{u}_{h}^{0}, \sigma_{h}^{0}\right)=\left(\Pi_{\mathbf{u}}\left(\mathbf{u}_{0}\right), \Pi_{\sigma}\left(\sigma_{0}\right)\right)$ and $\left(\mathbf{u}_{h}^{1}, \sigma_{h}^{1}\right)=$ $\left(\frac{\mathbf{u}_{h}^{2 / 3}+\mathbf{u}_{h}^{4 / 3}}{2}, \frac{\sigma_{h}^{2 / 3}+\sigma_{h}^{4 / 3}}{2}\right)$, where $\left(\mathbf{u}_{h}^{2 / 3}, \sigma_{h}^{2 / 3}\right)$ and $\left(\mathbf{u}_{h}^{4 / 3}, \sigma_{h}^{4 / 3}\right)$ are solutions of the first order backward Euler scheme with time step $\frac{2}{3} \Delta t$ at $t=\frac{2}{3} \Delta t$ and $t=\frac{4}{3} \Delta t$, respectively. For details, please see [20, 24]. Of course, we can follow the Crank-Nicolson/Adams-Bashforth scheme [40] for Navier-Stokes equations to obtain $\left(\mathbf{u}_{h}^{1}, \sigma_{h}^{1}\right)$.

\subsection{The existence and uniqueness of the numerical solution}

To ensure the computability of Scheme 3.2, we begin by showing that it is uniquely solvable for $\mathbf{u}_{h}$ and $\sigma_{h}$ at each time level.

Before proving the existence of solutions, we need to introduce the following induction hypothesis:

$$
\left\|\mathbf{u}_{h}^{n}\right\|_{\infty},\left\|\sigma_{h}^{n}\right\|_{\infty} \leq K . \quad \mathbf{I H 1}
$$

In Section 4, we will prove that the induction hypothesis IH1 is right for any $n=$ $0,1, \ldots, N$.

Lemma 3.4 Under the condition of hypothesis $\mathbf{I H 1}$, for $\Delta t \leq \min \left\{\frac{1-\alpha}{3 \operatorname{Red} C_{k}^{2} K^{2}}, \frac{3 \lambda \alpha(1-\alpha)}{72 d C_{k}^{2} K^{2}-2 \alpha(1-\alpha)}\right\}$, there exists a unique solution $\left(\mathbf{u}_{h}^{n+1}, \sigma_{h}^{n+1}, p_{h}^{n+1}\right) \in X_{h} \times S_{h} \times Q_{h}$ satisfying (3.1a)-(3.1c).

Proof Taking $\mathbf{v}_{h}=2 \alpha \mathbf{u}_{h}^{n+1}$ in (3.1a), $q_{h}=2 \alpha p_{h}^{n+1}$ in (3.1b) and $\tau_{h}=\sigma_{h}^{n+1}$ in (3.1c), adding together the three equations thus obtained, we deduce that

$$
\begin{aligned}
A\left(\mathbf{u}_{h}^{n+1}, \sigma_{h}^{n+1} ; \mathbf{u}_{h}^{n+1}, \sigma_{h}^{n+1}\right)= & \frac{2 \alpha R e}{2 \triangle t}\left(4 \mathbf{u}_{h}^{n}-\mathbf{u}_{h}^{n-1}, \mathbf{u}_{h}^{n+1}\right)+\frac{\lambda}{2 \triangle t}\left(4 \sigma_{h}^{n}, \sigma_{h}^{n+1}\right) \\
& -\frac{\lambda}{2 \triangle t}\left(\sigma_{h}^{n-1}, \sigma_{h}^{n+1}\right)+2 \alpha\left(\mathbf{f}^{n+1}, \mathbf{u}_{h}^{n+1}\right),
\end{aligned}
$$

where the bilinear form $A\left(\mathbf{u}_{h}^{n+1}, \sigma_{h}^{n+1} ; \mathbf{v}_{h}, \tau_{h}\right)$ is defined by

$$
\begin{aligned}
A\left(\mathbf{u}_{h}^{n+1}, \sigma_{h}^{n+1} ; \mathbf{v}_{h}, \tau_{h}\right)= & \frac{2 \alpha R e}{2 \triangle t}\left(3 \mathbf{u}_{h}^{n+1}, \mathbf{v}_{h}\right)+\frac{\lambda}{2 \triangle t}\left(3 \sigma_{h}^{n+1}, \tau_{h}\right)+\left(\sigma_{h}^{n+1}, \tau_{h}\right) \\
& +4 \alpha(1-\alpha)\left(D\left(\mathbf{u}_{h}^{n+1}\right), D\left(\mathbf{v}_{h}\right)\right)+2 \alpha \operatorname{Rec}\left(\digamma\left(\mathbf{u}_{h}^{n+1}\right), \mathbf{u}_{h}^{n+1}, \mathbf{v}_{h}\right) \\
& +\lambda\left(g_{a}\left(\digamma\left(\sigma_{h}^{n+1}\right), \nabla \mathbf{u}_{h}^{n+1}\right), \tau_{h}\right)+\lambda B\left(\digamma\left(\mathbf{u}_{h}^{n+1}\right), \sigma_{h}^{n+1}, \tau_{h}\right) .
\end{aligned}
$$

We now estimate the nonlinear terms on the right-hand sides (RHS) of $A\left(\mathbf{u}_{h}^{n+1}, \sigma_{h}^{n+1}\right.$; $\left.\mathbf{v}_{h}, \tau_{h}\right)$ in (3.5). In view of (2.8) and the Holder inequality, we deduce that

$$
\begin{aligned}
2 \alpha \operatorname{Re}\left|c\left(\digamma\left(\mathbf{u}_{h}^{n+1}\right), \mathbf{u}_{h}^{n+1}, \mathbf{u}_{h}^{n+1}\right)\right| & =2 \alpha \operatorname{Re}\left|\left(\digamma\left(\mathbf{u}_{h}^{n+1}\right) \cdot \nabla \mathbf{u}_{h}^{n+1}, \mathbf{u}_{h}^{n+1}\right)\right| \\
& \leq 2 \alpha \operatorname{Re} \sqrt{d}\left\|\digamma\left(\mathbf{u}_{h}^{n+1}\right)\right\|_{\infty}\left\|\nabla \mathbf{u}_{h}^{n+1}\right\|\left\|\mathbf{u}_{h}^{n+1}\right\| \\
& \leq 6 \alpha \operatorname{Re} \sqrt{d} K\left\|\nabla \mathbf{u}_{h}^{n+1}\right\|\left\|\mathbf{u}_{h}^{n+1}\right\| \\
& \leq \epsilon_{0}\left\|D\left(\mathbf{u}_{h}^{n+1}\right)\right\|^{2}+\frac{9 d \alpha^{2} \operatorname{Re}^{2} K^{2} C_{k}^{2}}{\epsilon_{0}}\left\|\mathbf{u}_{h}^{n+1}\right\|^{2},
\end{aligned}
$$




$$
\begin{aligned}
\lambda\left|\left(g_{a}\left(\digamma\left(\sigma_{h}^{n+1}\right), \nabla \mathbf{u}_{h}^{n+1}\right), \sigma_{h}^{n+1}\right)\right| & \leq 4\left\|\digamma\left(\sigma_{h}^{n+1}\right) \nabla \mathbf{u}_{h}^{n+1}\right\|\left\|\sigma_{h}^{n+1}\right\| \\
& \leq 4 \sqrt{d}\left\|\digamma\left(\sigma_{h}^{n+1}\right)\right\|\left\|_{\infty}\right\| \nabla \mathbf{u}_{h}^{n+1}\|\| \sigma_{h}^{n+1} \| \\
& \leq 4 \sqrt{d}\left(2\left\|\sigma_{h}^{n}\right\|_{\infty}+\left\|\sigma_{h}^{n-1}\right\|_{\infty}\right) C_{k}\left\|D\left(\mathbf{u}_{h}^{n+1}\right)\right\|\left\|\sigma_{h}^{n+1}\right\| \\
& \leq 12 \sqrt{d} C_{k} K\left\|D\left(\mathbf{u}_{h}^{n+1}\right)\right\|\left\|\sigma_{h}^{n+1}\right\| \\
& \leq \overline{\epsilon_{0}}\left\|D\left(\mathbf{u}_{h}^{n+1}\right)\right\|^{2}+\frac{36 d C_{k}^{2} K^{2}}{\bar{\epsilon}_{0}}\left\|\sigma_{h}^{n+1}\right\|^{2} .
\end{aligned}
$$

Note that $\lambda B\left(\digamma\left(\mathbf{u}_{h}^{n+1}\right), \sigma_{h}^{n+1}, \sigma_{h}^{n+1}\right)=\frac{\lambda}{2}\left\|\sigma_{h}^{n+1,+}-\sigma_{h}^{n+1,-}\right\|_{h, \digamma\left(\mathbf{u}_{h}^{n+1}\right)}^{2}$ due to the 'coercivity' (2.10) of $B(\cdot, \cdot, \cdot)$.

Combining the above inequalities with (3.5) yields

$$
\begin{aligned}
A\left(\mathbf{u}_{h}^{n+1}, \sigma_{h}^{n+1} ; \mathbf{u}_{h}^{n+1}, \sigma_{h}^{n+1}\right) \geq & \left(\frac{6 \alpha R e}{2 \triangle t}-\frac{9 d \alpha^{2} R e^{2} K^{2} C_{k}^{2}}{\epsilon_{0}}\right)\left\|\mathbf{u}_{h}^{n+1}\right\|^{2} \\
& +\left(\frac{3 \lambda}{2 \triangle t}-\frac{36 d C_{k}^{2} K^{2}}{\overline{\epsilon_{0}}}+1\right)\left\|\sigma_{h}^{n+1}\right\|^{2} \\
& +\left(4 \alpha(1-\alpha)-\epsilon_{0}-\overline{\epsilon_{0}}\right)\left\|D\left(\mathbf{u}_{h}^{n+1}\right)\right\|^{2} \\
& +\frac{\lambda}{2}\left\|\sigma_{h}^{n+1,+}-\sigma_{h}^{n+1,-}\right\|_{h, \digamma}^{2}\left(\mathbf{u}_{h}^{n+1}\right)
\end{aligned}
$$

Choose $\epsilon_{0}=\overline{\epsilon_{0}}=\alpha(1-\alpha)$ and

$$
\Delta t \leq \min \left\{\frac{1-\alpha}{3 \operatorname{Red} C_{k}^{2} K^{2}}, \frac{3 \lambda \alpha(1-\alpha)}{72 d C_{k}^{2} K^{2}-2 \alpha(1-\alpha)}\right\},
$$

thus the bilinear form $A\left(\mathbf{u}_{h}^{n+1}, \sigma_{h}^{n+1} ; \mathbf{v}_{h}, \tau_{h}\right)$ is positive. Since system (3.4) is a finite dimensional linear system, then the existence and uniqueness of solutions $\left(\mathbf{u}_{h}^{n+1}, \sigma_{h}^{n+1}, p_{h}^{n+1}\right)$ to Scheme 3.1 follow from the Lax-Milgram theorem and inf-sup condition (2.7).

\subsection{Numerical stability of Scheme 3.1}

Theorem 3.5 Suppose that $\mathbf{f} \in L^{2}\left(0, T ; H^{-1}(\Omega)^{d}\right)$, the initial value $\mathbf{u}_{0} \in L^{2}(\Omega)^{d}$ and $\sigma_{0} \in$ $L^{2}(\Omega)^{d \times d}$. For time step $\Delta t$ small enough, Scheme 3.1 is stable and satisfying

$$
\begin{aligned}
& \alpha \operatorname{Re}\left\|\mathbf{u}_{h}^{l}\right\|^{2}+\frac{\lambda}{2}\left\|\sigma_{h}^{l}\right\|^{2}+\Delta t \sum_{n=0}^{l-1}\left[2 \alpha(1-\alpha)\left\|D\left(\mathbf{u}_{h}^{n+1}\right)\right\|^{2}+2\left\|\sigma_{h}^{n+1}\right\|^{2}\right] \\
& \leq \exp \left(2 T \gamma_{n}\right)\left[\frac{2 C_{k}^{2} \alpha}{(1-\alpha)} \Delta t \sum_{n=0}^{l-1}\left\|\mathbf{f}^{n+1}\right\|_{-1}^{2}+2 \alpha \operatorname{Re}\left\|\mathbf{u}_{0}\right\|^{2}+\lambda\left\|\sigma_{0}\right\|^{2}\right] \\
& \beta^{2} \Delta t \sum_{n=0}^{l-1}\left\|p_{h}^{n+1}\right\|^{2} \leq C\left(\operatorname{Re}, \alpha, d, \lambda, T, \Omega, K, \mathbf{f}, \mathbf{u}_{0}, \sigma_{0}\right) .
\end{aligned}
$$

Proof Choosing $\mathbf{v}_{h}=2 \Delta t \mathbf{u}_{h}^{n+1}$ in (3.1a), $q_{h}=2 \Delta t p_{h}^{n+1}$ in (3.1b) and $\tau_{h}=2 \Delta t \sigma_{h}^{n+1}$ in (3.1c), we get

$$
\begin{aligned}
& 2 \operatorname{Re} \Delta t\left(\beth\left(\mathbf{u}_{h}^{n+1}\right), \mathbf{u}_{h}^{n+1}\right)+2 \operatorname{Re} \triangle t c\left(\digamma\left(\mathbf{u}_{h}^{n+1}\right), \mathbf{u}_{h}^{n+1}, \mathbf{u}_{h}^{n+1}\right) \\
& \quad+2 \Delta t\left(\sigma_{h}^{n+1}, D\left(\mathbf{u}_{h}^{n+1}\right)\right)+4(1-\alpha) \triangle t\left\|D\left(\mathbf{u}_{h}^{n+1}\right)\right\|^{2}=2 \Delta t\left(\mathbf{f}^{n+1}, \mathbf{u}_{h}^{n+1}\right),
\end{aligned}
$$




$$
\begin{aligned}
2 \lambda & \Delta t\left(\beth\left(\sigma_{h}^{n+1}\right), \sigma_{h}^{n+1}\right)+2 \Delta t\left\|\sigma_{h}^{n+1}\right\|^{2}+2 \lambda \triangle t\left(g_{a}\left(\digamma\left(\sigma_{h}^{n+1}\right), \nabla \mathbf{u}_{h}^{n+1}\right), \sigma_{h}^{n+1}\right) \\
+ & \Delta t \lambda\left\|\sigma_{h}^{n+1,+}-\sigma_{h}^{n+1,-}\right\|_{h, \digamma\left(\mathbf{u}_{h}^{n+1}\right)}^{2}-4 \alpha \Delta t\left(D\left(\mathbf{u}_{h}^{n+1}\right), \sigma_{h}^{n+1}\right)=0 .
\end{aligned}
$$

Multiplying (3.9) by $2 \alpha$ and adding to (3.10) yield the single equation

$$
\begin{aligned}
4 \alpha \operatorname{Re} & \triangle t\left(\beth\left(\mathbf{u}_{h}^{n+1}\right), \mathbf{u}_{h}^{n+1}\right)+4 \operatorname{Re} \alpha \Delta t c\left(\digamma\left(\mathbf{u}_{h}^{n+1}\right), \mathbf{u}_{h}^{n+1}, \mathbf{u}_{h}^{n+1}\right) \\
& +2 \lambda \triangle t\left(\beth\left(\sigma_{h}^{n+1}\right), \sigma_{h}^{n+1}\right)+8 \alpha(1-\alpha) \triangle t\left\|D\left(\mathbf{u}_{h}^{n+1}\right)\right\|^{2}+2 \triangle t\left\|\sigma_{h}^{n+1}\right\|^{2} \\
& +2 \lambda \triangle t\left(g_{a}\left(\digamma\left(\sigma_{h}^{n+1}\right), \nabla \mathbf{u}_{h}^{n+1}\right), \sigma_{h}^{n+1}\right)+\Delta t \lambda\left\|\sigma_{h}^{n+1,+}-\sigma_{h}^{n+1,-}\right\|_{h, \digamma\left(u_{h}^{n+1}\right)}^{2} \\
= & 4 \alpha \Delta t\left(\mathbf{f}^{n+1}, \mathbf{u}_{h}^{n+1}\right) .
\end{aligned}
$$

Furthermore, we have

$$
\begin{aligned}
4 R e \alpha & \triangle t\left|c\left(\digamma\left(\mathbf{u}_{h}^{n+1}\right), \mathbf{u}_{h}^{n+1}, \mathbf{u}_{h}^{n+1}\right)\right| \\
& =4 \operatorname{Re} \alpha \Delta t \mid\left(\left(\digamma\left(\mathbf{u}_{h}^{n+1}\right) \cdot \nabla \mathbf{u}_{h}^{n+1}, \mathbf{u}_{h}^{n+1}\right) \mid\right. \\
& \leq 4 \operatorname{Re} \alpha \Delta t\left\|\digamma\left(\mathbf{u}_{h}^{n+1}\right) \cdot \nabla \mathbf{u}_{h}^{n+1}\right\|\left\|\mathbf{u}_{h}^{n+1}\right\| \\
& \leq 4 \operatorname{Re} \alpha \sqrt{d} C_{k} \Delta t\left\|\digamma\left(\mathbf{u}_{h}^{n+1}\right)\right\|_{\infty}\left\|D\left(\mathbf{u}_{h}^{n+1}\right)\right\|\left\|\mathbf{u}_{h}^{n+1}\right\| \\
& \leq 12 \operatorname{Re} \alpha \sqrt{d} K C_{k} \triangle t\left\|D\left(\mathbf{u}_{h}^{n+1}\right)\right\|\left\|\mathbf{u}_{h}^{n+1}\right\| \\
& \leq \epsilon_{1} \triangle t\left\|D\left(\mathbf{u}_{h}^{n+1}\right)\right\|^{2}+\frac{36 \operatorname{Re}^{2} \alpha^{2} d C_{k}^{2} K^{2}}{\epsilon_{1}} \Delta t\left\|\mathbf{u}_{h}^{n+1}\right\|^{2}, \\
2 \lambda & \triangle t\left|\left(g_{a}\left(\digamma\left(\sigma_{h}^{n+1}\right), \nabla \mathbf{u}_{h}^{n+1}\right), \sigma_{h}^{n+1}\right)\right| \\
& \leq 8 \lambda \triangle t \sqrt{d}\left\|\digamma\left(\sigma_{h}^{n+1}\right)\right\|\left\|_{\infty}\right\| \nabla \mathbf{u}_{h}^{n+1}\|\| \sigma_{h}^{n+1} \| \\
& \leq 24 \lambda \triangle t \sqrt{d} C_{k} K\left\|D\left(\mathbf{u}_{h}^{n+1}\right)\right\|\left\|\sigma_{h}^{n+1}\right\| \\
& \leq \Delta t \overline{\epsilon_{1}}\left\|D\left(\mathbf{u}_{h}^{n+1}\right)\right\|^{2}+\Delta t \frac{144 \lambda^{2} d C_{k}^{2} K^{2}}{\bar{\epsilon}_{1}}\left\|\sigma_{h}^{n+1}\right\|^{2}
\end{aligned}
$$

and

$$
\begin{aligned}
\alpha \Delta t\left|\left(\mathbf{f}^{n+1}, \mathbf{u}_{h}^{n+1}\right)\right| & \leq 4 \alpha \Delta t\left\|\mathbf{f}^{n+1}\right\|_{-1}\left\|\nabla \mathbf{u}_{h}^{n+1}\right\| \\
& \leq 4 \alpha C_{k} \Delta t\left\|\mathbf{f}^{n+1}\right\|_{-1}\left\|D\left(\mathbf{u}_{h}^{n+1}\right)\right\| \\
& \leq \Delta t \overline{\bar{\epsilon}}_{1}\left\|D\left(\mathbf{u}_{h}^{n+1}\right)\right\|^{2}+\Delta t \frac{4 C_{k}^{2} \alpha^{2}}{\overline{\bar{\epsilon}}_{1}}\left\|\mathbf{f}^{n+1}\right\|_{-1}^{2} .
\end{aligned}
$$

Plugging (3.12)-(3.14) into (3.11) and setting $\epsilon_{1}=\bar{\epsilon}_{1}=\overline{\bar{\epsilon}}_{1}=2 \alpha(1-\alpha)$ yield

$$
\begin{aligned}
4 \alpha \operatorname{Re} & \triangle t\left(\beth\left(\mathbf{u}_{h}^{n+1}\right), \mathbf{u}_{h}^{n+1}\right)+2 \lambda \triangle t\left(\beth\left(\sigma_{h}^{n+1}\right), \sigma_{h}^{n+1}\right) \\
& +2 \alpha(1-\alpha) \triangle t\left\|D\left(\mathbf{u}_{h}^{n+1}\right)\right\|^{2}+2 \triangle t\left\|\sigma_{h}^{n+1}\right\|^{2} \\
\leq & \frac{72 \lambda^{2} d C_{k}^{2} K^{2}}{\alpha(1-\alpha)} \Delta t\left\|\sigma_{h}^{n+1}\right\|^{2}+\frac{18 e^{2} d C_{k}^{2} K^{2}}{\alpha(1-\alpha)} \Delta t\left\|\mathbf{u}_{h}^{n+1}\right\|^{2} \\
& +\Delta t \frac{2 C_{k}^{2} \alpha}{(1-\alpha)}\left\|\mathbf{f}^{n+1}\right\|_{-1}^{2} .
\end{aligned}
$$


Using identity (2.13) to (3.15), we get

$$
\begin{aligned}
& \alpha \operatorname{Re}\left[3\left\|\mathbf{u}_{h}^{n+1}\right\|^{2}-4\left\|\mathbf{u}_{h}^{n}\right\|^{2}+\left\|\mathbf{u}_{h}^{n-1}\right\|^{2}\right]+2 \alpha \operatorname{Re}\left[\left\|\mathbf{u}_{h}^{n+1}-\mathbf{u}_{h}^{n}\right\|^{2}-\left\|\mathbf{u}_{h}^{n}-\mathbf{u}_{h}^{n-1}\right\|^{2}\right] \\
&+\alpha \operatorname{Re}\left\|\mathbf{u}_{h}^{n+1}-2 \mathbf{u}_{h}^{n}+\mathbf{u}_{h}^{n-1}\right\|^{2}+\frac{\lambda}{2}\left[3\left\|\sigma_{h}^{n+1}\right\|^{2}-4\left\|\sigma_{h}^{n}\right\|^{2}+\left\|\sigma_{h}^{n-1}\right\|^{2}\right] \\
&+\lambda\left[\left\|\sigma_{h}^{n+1}-\sigma_{h}^{n}\right\|^{2}-\left\|\sigma_{h}^{n}-\sigma_{h}^{n-1}\right\|^{2}\right]+2 \Delta t\left\|\sigma_{h}^{n+1}\right\|^{2} \\
& \quad+\frac{\lambda}{2}\left\|\sigma_{h}^{n+1}-2 \sigma_{h}^{n}+\sigma_{h}^{n-1}\right\|^{2}+2 \alpha(1-\alpha) \Delta t\left\|D\left(\mathbf{u}_{h}^{n+1}\right)\right\|^{2} \\
& \leq \frac{72 \lambda^{2} d C_{k}^{2} K^{2}}{\alpha(1-\alpha)} \Delta t\left\|\sigma_{h}^{n+1}\right\|^{2}+\frac{18 e^{2} d C_{k}^{2} K^{2}}{\alpha(1-\alpha)} \Delta t\left\|\mathbf{u}_{h}^{n+1}\right\|^{2} \\
&+\Delta t \frac{2 C_{k}^{2} \alpha}{(1-\alpha)}\left\|\mathbf{f}^{n+1}\right\|_{-1}^{2} .
\end{aligned}
$$

Summing (3.16) from $n=0$ to $l-1$ and using the identity

$$
\frac{3}{2} a^{2}-\frac{1}{2} b^{2}+(a-b)^{2}=\frac{1}{2} a^{2}+\left(\sqrt{2} a-\frac{b}{\sqrt{2}}\right)^{2}
$$

to $(3.16)$ yield

$$
\begin{aligned}
& \alpha \operatorname{Re}\left[\left\|\mathbf{u}_{h}^{l}\right\|^{2}+\left\|2 \mathbf{u}_{h}^{l}-\mathbf{u}_{h}^{l-1}\right\|^{2}\right]+\frac{\lambda}{2}\left[\left\|\sigma_{h}^{l}\right\|^{2}+\left\|2 \sigma_{h}^{l}-\sigma_{h}^{l-1}\right\|^{2}\right] \\
& +2 \alpha(1-\alpha) \Delta t \sum_{n=0}^{l-1}\left\|D\left(\mathbf{u}_{h}^{n+1}\right)\right\|^{2}+2 \Delta t \sum_{n=0}^{l-1}\left\|\sigma_{h}^{n+1}\right\|^{2} \\
& \leq \frac{18 \operatorname{Re}^{2} d C_{k}^{2} K^{2}}{\alpha(1-\alpha)} \Delta t \sum_{n=0}^{l-1}\left\|\mathbf{u}_{h}^{n+1}\right\|^{2}+\frac{72 \lambda^{2} d C_{k}^{2} K^{2}}{\alpha(1-\alpha)} \Delta t \sum_{n=0}^{l-1}\left\|\sigma_{h}^{n+1}\right\|^{2} \\
& +\Delta t \frac{2 C_{k}^{2} \alpha}{(1-\alpha)} \sum_{n=0}^{l-1}\left\|\mathbf{f}^{n+1}\right\|_{-1}^{2}+2 \alpha \operatorname{Re}\left\|\mathbf{u}_{0}\right\|^{2}+\lambda\left\|\sigma_{0}\right\|^{2} .
\end{aligned}
$$

In order to use the discrete Gronwall Lemma 2.3, here we set

$$
\begin{aligned}
& a_{l}=\alpha \operatorname{Re}\left\|\mathbf{u}_{h}^{l}\right\|^{2}+\frac{\lambda}{2}\left\|\sigma_{h}^{l}\right\|^{2}, \quad b_{n}=2 \alpha(1-\alpha)\left\|D\left(\mathbf{u}_{h}^{n+1}\right)\right\|^{2}+2\left\|\sigma_{h}^{n+1}\right\|^{2}, \\
& a_{n}=\alpha \operatorname{Re}\left\|\mathbf{u}_{h}^{n+1}\right\|^{2}+\frac{\lambda}{2}\left\|\sigma_{h}^{n+1}\right\|^{2}, \\
& \gamma_{n}=\max \left\{\frac{18 \operatorname{Red} C_{k}^{2} K^{2}}{\alpha^{2}(1-\alpha)}, \frac{144 \lambda d C_{k}^{2} K^{2}}{\alpha(1-\alpha)}\right\}, \quad \zeta_{n}=\frac{1}{1-\Delta t \gamma_{n}}, \\
& c_{n}=\frac{2 C_{k}^{2} \alpha}{(1-\alpha)}\left\|\mathbf{f}^{n+1}\right\|_{-1}^{2}, \quad H=2 \alpha \operatorname{Re}\left\|\mathbf{u}_{0}\right\|^{2}+\lambda\left\|\sigma_{0}\right\|^{2} .
\end{aligned}
$$

For time step $\Delta t$ such that $\gamma_{n} \Delta t \leq \frac{1}{2}$, thus using the discrete Gronwall lemma to (3.18) yields the result (3.7).

Now we bound the pressure. As $V_{h} \subset X_{h}$, for all $\mathbf{v}_{h} \in V_{h}$, we have from (3.2a)

$$
\begin{aligned}
& \operatorname{Re}\left(\beth\left(\mathbf{u}_{h}^{n+1}\right), \mathbf{v}_{h}\right)+\operatorname{Rec}\left(\digamma\left(\mathbf{u}_{h}^{n+1}\right), \mathbf{u}_{h}^{n+1}, \mathbf{v}_{h}\right)+\left(\sigma_{h}^{n+1}, D\left(\mathbf{v}_{h}\right)\right) \\
& \quad+(1-\alpha)\left(\nabla \mathbf{u}_{h}^{n+1}, \nabla \mathbf{v}_{h}\right)=\left(\mathbf{f}^{n+1}, \mathbf{v}_{h}\right) .
\end{aligned}
$$


Dividing by $\left\|\nabla \mathbf{v}_{h}\right\|$ and using the Cauchy-Schwarz inequality, we get

$$
\begin{aligned}
\operatorname{Re} \frac{\left(\beth\left(\mathbf{u}_{h}^{n+1}\right), \mathbf{v}_{h}\right)}{\left\|\nabla \mathbf{v}_{h}\right\|} \leq & \operatorname{Re} \sqrt{d} C_{p}\left\|\digamma\left(\mathbf{u}_{h}^{n+1}\right)\right\|_{\infty}\left\|\nabla \mathbf{u}_{h}^{n+1}\right\|+\left\|\sigma_{h}^{n+1}\right\| \\
& +(1-\alpha)\left\|\nabla \mathbf{u}_{h}^{n+1}\right\|+\left\|\mathbf{f}^{n+1}\right\|_{-1} \cdot
\end{aligned}
$$

Taking the supremum over $\mathbf{v}_{h} \in V_{h}$ yields

$$
\begin{aligned}
\operatorname{Re}\left\|\beth\left(\mathbf{u}_{h}^{n+1}\right)\right\|_{V_{h}^{\prime}} \leq & \left(3 \operatorname{Re} \sqrt{d} C_{p} K+1-\alpha\right)\left\|\nabla \mathbf{u}_{h}^{n+1}\right\|+\left\|\sigma_{h}^{n+1}\right\| \\
& +\left\|\mathbf{f}^{n+1}\right\|_{-1} .
\end{aligned}
$$

The bound along with Lemma 2.2 provides the following estimate:

$$
\begin{aligned}
\operatorname{Re}\left\|\beth\left(\mathbf{u}_{h}^{n+1}\right)\right\|_{X_{h}^{\prime}} \leq & \left(3 \operatorname{Re} \sqrt{d} C_{p} K+1-\alpha\right)\left\|\nabla \mathbf{u}_{h}^{n+1}\right\|+\left\|\sigma_{h}^{n+1}\right\| \\
& +\left\|\mathbf{f}^{n+1}\right\|_{-1} \cdot
\end{aligned}
$$

From (3.1a) we have

$$
\begin{aligned}
\left(\nabla \cdot \mathbf{v}_{h}, p_{h}^{n+1}\right)= & \operatorname{Re}\left(\beth\left(\mathbf{u}_{h}^{n+1}\right), \mathbf{v}_{h}\right)+\operatorname{Rec}\left(\digamma\left(\mathbf{u}_{h}^{n+1}\right), \mathbf{u}_{h}^{n+1}, \mathbf{v}_{h}\right) \\
& +\left(\sigma_{h}^{n+1}, D\left(\mathbf{v}_{h}\right)\right)+(1-\alpha)\left(\nabla \mathbf{u}_{h}^{n+1}, \nabla \mathbf{v}_{h}\right)-\left(\mathbf{f}^{n+1}, \mathbf{v}_{h}\right)
\end{aligned}
$$

Dividing by $\left\|\nabla \mathbf{v}_{h}\right\|$ and using the Cauchy-Schwarz inequality yield

$$
\begin{aligned}
\frac{\left(\nabla \cdot \mathbf{v}_{h}, p_{h}^{n+1}\right)}{\left\|\nabla \mathbf{v}_{h}\right\|} \leq & \operatorname{Re} \frac{\left(\beth\left(\mathbf{u}_{h}^{n+1}\right), \mathbf{v}_{h}\right)}{\left\|\nabla \mathbf{v}_{h}\right\|}+\left(3 \operatorname{Re} \sqrt{d} C_{p} K+1-\alpha\right)\left\|\nabla \mathbf{u}_{h}^{n+1}\right\| \\
& +\left\|\sigma_{h}^{n+1}\right\|+\left\|\mathbf{f}^{n+1}\right\|_{-1} .
\end{aligned}
$$

Taking the supremum over $\mathbf{v}_{h} \in X_{h}$ and using the inf-sup conditions (2.7), we have

$$
\begin{aligned}
\beta\left\|p_{h}^{n+1}\right\| \leq & \operatorname{Re}\left\|\beth\left(\mathbf{u}_{h}^{n+1}\right)\right\|_{X_{h}^{\prime}}+\left(3 \operatorname{Re} \sqrt{d} C_{p} K+1-\alpha\right)\left\|\nabla \mathbf{u}_{h}^{n+1}\right\| \\
& +\left\|\sigma_{h}^{n+1}\right\|+\left\|\mathbf{f}^{n+1}\right\|_{-1} \\
\leq & \left(6 \operatorname{Re} \sqrt{d} C_{p} K+2-2 \alpha\right)\left\|\nabla \mathbf{u}_{h}^{n+1}\right\|+2\left\|\sigma_{h}^{n+1}\right\|+2\left\|\mathbf{f}^{n+1}\right\|_{-1} .
\end{aligned}
$$

Applying $(a+b+c)^{2} \leq 3\left(a^{2}+b^{2}+c^{2}\right)$ to (3.19) yields

$$
\beta^{2}\left\|p_{h}^{n+1}\right\|^{2} \leq 3\left(6 \operatorname{Re} \sqrt{d} C_{p} K+2-2 \alpha\right)^{2}\left\|\nabla \mathbf{u}_{h}^{n+1}\right\|^{2}+12\left\|\sigma_{h}^{n+1}\right\|^{2}+12\left\|\mathbf{f}^{n+1}\right\|_{-1}^{2} .
$$

Now multiplying by $\Delta t$, summing over $n$ from 0 to $l-1$ and using the bound (3.7), we get the required result (3.8).

\section{Error analysis of BDF2-LE scheme}

We proceed to give an a priori error estimate for the BDF2-LE Galerkin FEM. In order to simplify the descriptions, we denote

$$
e_{\mathbf{u}}^{n}=\mathbf{u}^{n}-\mathbf{u}_{h}^{n}, \quad e_{p}^{n}=p^{n}-p_{h}^{n}, \quad e_{\sigma}^{n}=\sigma^{n}-\sigma_{h}^{n},
$$


where $\left(\mathbf{u}^{n}, p^{n}, \sigma^{n}\right)$ and $\left(\mathbf{u}_{h}^{n}, p_{h}^{n}, \sigma_{h}^{n}\right)$ are the solutions of problems (2.1a)-(2.1c) and (3.1a)(3.1c), respectively. We construct the error equations for velocity $e_{\mathbf{u}}^{n}$, pressure $e_{p}^{n}$ and stress tensor $e_{\sigma}^{n}$. Decompose

$$
\begin{aligned}
& e_{\mathbf{u}}^{n}=\left(\mathbf{u}^{n}-\Pi_{\mathbf{u}}\left(\mathbf{u}^{n}\right)\right)+\left(\Pi_{\mathbf{u}}\left(\mathbf{u}^{n}\right)-\mathbf{u}_{h}^{n}\right)=\eta_{\mathbf{u}}^{n}+\varphi_{\mathbf{u}}^{n}, \\
& e_{p}^{n}=\left(p^{n}-\Pi_{p}\left(p^{n}\right)\right)+\left(\Pi_{p}\left(p^{n}\right)-p_{h}^{n}\right)=\eta_{p}^{n}+\varphi_{p}^{n}, \\
& e_{\sigma}^{n}=\left(\sigma^{n}-\Pi_{\sigma}\left(\sigma^{n}\right)\right)+\left(\Pi_{\sigma}\left(\sigma^{n}\right)-\sigma_{h}^{n}\right)=\eta_{\sigma}^{n}+\varphi_{\sigma}^{n},
\end{aligned}
$$

where $\left(\Pi_{\mathbf{u}}(\mathbf{u}), \Pi_{p}(p), \Pi_{\sigma}(\sigma)\right)$ denote the elements in $X_{h} \times Q_{h} \times S_{h}$ and satisfy the approximation properties (2.4)-(2.6). To establish the error estimate, we introduce the following discrete norms:

$$
\|\omega\|_{\infty, k}=\max _{0 \leq n \leq N-1}\left\|\omega^{n+1}\right\|_{k}, \quad\|\omega\|_{0, k}=\left[\Delta t \sum_{n=0}^{N-1}\left\|\omega^{n+1}\right\|_{k}^{2}\right]^{\frac{1}{2}} .
$$

Theorem 4.1 Suppose that $(\mathbf{u}, p, \sigma)$ is a weak solution to (2.1a)-(2.1c) with additional regularities (2.3). $\left(\mathbf{u}_{h}^{l}, p_{h}^{l}, \sigma_{h}^{l}\right)$ is given by (3.1a)-(3.1c) for $l \in\{0,1, \ldots, N-1\}$. For hypothesis IH1 and $\triangle t \gamma_{n} \leq \frac{1}{2}$, we have

$$
\begin{gathered}
\alpha \operatorname{Re}\left\|\varphi_{\mathbf{u}}^{l+1}\right\|^{2}+\alpha \operatorname{Re}\left\|2 \varphi_{\mathbf{u}}^{l+1}-\varphi_{\mathbf{u}}^{l}\right\|^{2}+\frac{\lambda}{2}\left\|\varphi_{\sigma}^{l+1}\right\|^{2}+\frac{\lambda}{2}\left\|2 \varphi_{\sigma}^{l+1}-\varphi_{\sigma}^{l}\right\|^{2} \\
+4 \alpha(1-\alpha) \Delta t \sum_{n=0}^{l}\left\|D\left(\varphi_{\mathbf{u}}^{n+1}\right)\right\|^{2}+\Delta t \sum_{n=0}^{l}\left\|\varphi_{\sigma}^{n+1}\right\|^{2} \leq W(\Delta t, h),
\end{gathered}
$$

where

$$
\gamma_{n}=\max \left\{1, \frac{d^{2} M^{2}}{\alpha}\left(20+\frac{13 \lambda^{2} d}{R e}\right), \frac{d^{2} C_{k}^{2} \lambda}{\alpha(1-\alpha)}\left(\frac{15 d M^{2}}{2}+432 K^{2}\right), 416 \lambda d^{2} M^{2}\right\}
$$

and

$$
\begin{aligned}
W(\triangle t, h)= & \exp \left(2 T \gamma_{n}\right)\left[( \Delta t ) ^ { 4 } \left(\left\|\partial_{t}^{3} \mathbf{u}\right\|_{L^{2}\left(0, T ; L^{2}(\Omega)^{d}\right)}^{2}+\left\|\partial_{t}^{3} \sigma\right\|_{L^{2}\left(0, T ; L^{2}(\Omega)^{d \times d}\right)}^{2}\right.\right. \\
& \left.+\left\|\partial_{t}^{2} \mathbf{u}\right\|_{L^{2}\left(0, T ; L^{2}(\Omega)^{d}\right)}^{2}+\left\|\partial_{t}^{2} \sigma\right\|_{L^{2}\left(0, T ; L^{2}(\Omega) d \times d\right)}^{2}\right) \\
& +\left(h^{2 q+2}\|p\|_{0, q+1}^{2}+h^{2 m}\|\sigma\|_{0, m+1}^{2}+h^{2 k}\|\mathbf{u}\|_{0, k+1}^{2}\right. \\
& \left.\left.+h^{2 k}\left\|\partial_{t} \mathbf{u}\right\|_{L^{2}\left(0, T ; H^{k+1}(\Omega)^{d}\right)}^{2}+h^{2 m}\left\|\partial_{t} \sigma\right\|_{L^{2}\left(0, T ; H^{m+1}(\Omega)^{d \times d}\right)}^{2}\right)\right] .
\end{aligned}
$$

Proof At time $t_{n+1}=(n+1) \Delta t$, the true solution $(\mathbf{u}, p, \sigma)$ of $(2.2 \mathrm{a})-(2.2 \mathrm{~b})$ satisfies

$$
\begin{aligned}
& \operatorname{Re}\left(\beth\left(\mathbf{u}^{n+1}\right), \mathbf{v}_{h}\right)+\operatorname{Rec}\left(\digamma\left(\mathbf{u}^{n+1}\right), \mathbf{u}^{n+1}, \mathbf{v}_{h}\right)+\left(\sigma^{n+1}, D\left(\mathbf{v}_{h}\right)\right) \\
& \quad+2(1-\alpha)\left(D\left(\mathbf{u}^{n+1}\right), D\left(\mathbf{v}_{h}\right)\right) \\
&= \operatorname{Re}\left(\beth\left(\mathbf{u}^{n+1}\right)-\partial_{t} \mathbf{u}^{n+1}, \mathbf{v}_{h}\right) \\
&+\left(\mathbf{f}^{n+1}, \mathbf{v}_{h}\right)+\left(p^{n+1}, \nabla \cdot \mathbf{v}_{h}\right)+\operatorname{Rec}\left(\digamma\left(\mathbf{u}^{n+1}\right)-\mathbf{u}^{n+1}, \mathbf{u}^{n+1}, \mathbf{v}_{h}\right),
\end{aligned}
$$




$$
\begin{aligned}
\lambda(\beth & \left.\left(\sigma^{n+1}\right), \tau_{h}\right)+\left(\sigma^{n+1}, \tau_{h}\right)+\lambda B\left(\digamma\left(\mathbf{u}^{n+1}\right), \sigma^{n+1}, \tau_{h}\right) \\
& -2 \alpha\left(D\left(\mathbf{u}^{n+1}\right), \tau_{h}\right)+\lambda\left(g_{a}\left(\digamma\left(\sigma^{n+1}\right), \nabla \mathbf{u}^{n+1}\right), \tau_{h}\right) \\
= & \lambda\left(\beth\left(\sigma^{n+1}\right)-\partial_{t} \sigma^{n+1}, \tau_{h}\right)+\lambda B\left(\digamma\left(\mathbf{u}^{n+1}\right)-\mathbf{u}^{n+1}, \sigma^{n+1}, \tau_{h}\right) \\
& +\lambda\left(g_{a}\left(\digamma\left(\sigma^{n+1}\right)-\sigma^{n+1}, \nabla \mathbf{u}^{n+1}\right), \tau_{h}\right)
\end{aligned}
$$

for all $\left(\mathbf{v}_{h}, \tau_{h}\right) \in V_{h} \times S_{h}$. Subtract (4.5a)-(4.5b) from (3.2a)-(3.2b) to yield the following error equations for $e_{\mathbf{u}}$ and $e_{\sigma}$ :

$$
\begin{aligned}
\operatorname{Re}(\beth & \left.\left(e_{\mathbf{u}}^{n+1}\right), \mathbf{v}_{h}\right)+\left(e_{\sigma}^{n+1}, D\left(\mathbf{v}_{h}\right)\right)+2(1-\alpha)\left(D\left(e_{\mathbf{u}}^{n+1}\right), D\left(\mathbf{v}_{h}\right)\right) \\
& +\operatorname{Rec}\left(\digamma\left(\mathbf{u}^{n+1}\right), \mathbf{u}^{n+1}, \mathbf{v}_{h}\right)-\operatorname{Rec}\left(\digamma\left(\mathbf{u}_{h}^{n+1}\right), \mathbf{u}_{h}^{n+1}, \mathbf{v}_{h}\right) \\
= & \left(p^{n+1}, \nabla \cdot \mathbf{v}_{h}\right)+\operatorname{Re}\left(\beth\left(\mathbf{u}^{n+1}\right)-\partial_{t} \mathbf{u}^{n+1}, \mathbf{v}_{h}\right) \\
& +\operatorname{Rec}\left(\digamma\left(\mathbf{u}^{n+1}\right)-\mathbf{u}^{n+1}, \mathbf{u}^{n+1}, \mathbf{v}_{h}\right), \\
\lambda\left(\beth\left(e_{\sigma}^{n+1}\right), \tau_{h}\right)+\left(e_{\sigma}^{n+1}, \tau_{h}\right)-2 \alpha\left(D\left(e_{\mathbf{u}}^{n+1}\right), \tau_{h}\right) & +\lambda B\left(\digamma\left(\mathbf{u}^{n+1}\right), \sigma^{n+1}, \tau_{h}\right)-\lambda B\left(\digamma\left(\mathbf{u}_{h}^{n+1}\right), \sigma_{h}^{n+1}, \tau_{h}\right) \\
& +\lambda\left(g_{a}\left(\digamma\left(\sigma^{n+1}\right), \nabla \mathbf{u}^{n+1}\right), \tau_{h}\right)-\lambda\left(g_{a}\left(\digamma\left(\sigma_{h}^{n+1}\right), \nabla \mathbf{u}_{h}^{n+1}\right), \tau_{h}\right) \\
= & \lambda\left(\beth\left(\sigma^{n+1}\right)-\partial_{t} \sigma^{n+1}, \tau_{h}\right)+\lambda B\left(\digamma\left(\mathbf{u}^{n+1}\right)-\mathbf{u}^{n+1}, \sigma^{n+1}, \tau_{h}\right) \\
& +\lambda\left(g_{a}\left(\digamma\left(\sigma^{n+1}\right)-\sigma^{n+1}, \nabla \mathbf{u}^{n+1}\right), \tau_{h}\right) .
\end{aligned}
$$

Taking $\mathbf{v}_{h}=\varphi_{\mathbf{u}}^{n+1}$ in (4.6a) and $\tau_{h}=\varphi_{\sigma}^{n+1}$ in (4.6b) yields

$$
\begin{aligned}
& \operatorname{Re}\left(\beth\left(\varphi_{\mathbf{u}}^{n+1}\right), \varphi_{\mathbf{u}}^{n+1}\right)+2(1-\alpha)\left(D\left(\varphi_{\mathbf{u}}^{n+1}\right), D\left(\varphi_{\mathbf{u}}^{n+1}\right)\right) \\
& \quad+\left(\varphi_{\sigma}^{n+1}, D\left(\varphi_{\mathbf{u}}^{n+1}\right)\right)+\operatorname{Rec}\left(\digamma\left(\mathbf{u}_{h}^{n+1}\right), \varphi_{\mathbf{u}}^{n+1}, \varphi_{\mathbf{u}}^{n+1}\right)=F_{1}\left(\varphi_{\mathbf{u}}^{n+1}\right), \\
& \lambda\left(\beth\left(\varphi_{\sigma}^{n+1}\right), \varphi_{\sigma}^{n+1}\right)+\left(\varphi_{\sigma}^{n+1}, \varphi_{\sigma}^{n+1}\right)-2 \alpha\left(D\left(\varphi_{\mathbf{u}}^{n+1}\right), \varphi_{\sigma}^{n+1}\right) \\
& \quad+\lambda B\left(\digamma\left(\mathbf{u}_{h}^{n+1}\right), \varphi_{\sigma}^{n+1}, \varphi_{\sigma}^{n+1}\right)=F_{2}\left(\varphi_{\sigma}^{n+1}\right),
\end{aligned}
$$

where

$$
\begin{aligned}
F_{1}\left(\varphi_{\mathbf{u}}^{n+1}\right)= & \operatorname{Re}\left(\beth\left(\Pi_{\mathbf{u}}\left(\mathbf{u}^{n+1}\right)\right)-\partial_{t} \mathbf{u}^{n+1}, \varphi_{\mathbf{u}}^{n+1}\right)+\left(p^{n+1}, \nabla \cdot \varphi_{\mathbf{u}}^{n+1}\right) \\
& -\left(\eta_{\sigma}^{n+1}, D\left(\varphi_{\mathbf{u}}^{n+1}\right)\right)-2(1-\alpha)\left(D\left(\eta_{\mathbf{u}}^{n+1}\right), D\left(\varphi_{\mathbf{u}}^{n+1}\right)\right) \\
& +\operatorname{Rec}\left(\digamma\left(\mathbf{u}^{n+1}\right)-\mathbf{u}^{n+1}, \mathbf{u}^{n+1}, \varphi_{\mathbf{u}}^{n+1}\right) \\
& -\operatorname{Rec}\left(\digamma\left(\varphi_{\mathbf{u}}^{n+1}\right), \mathbf{u}^{n+1}, \varphi_{\mathbf{u}}^{n+1}\right)-\operatorname{Rec}\left(\digamma\left(\eta_{\mathbf{u}}^{n+1}\right), \mathbf{u}^{n+1}, \varphi_{\mathbf{u}}^{n+1}\right) \\
& -\operatorname{Rec}\left(\digamma\left(\mathbf{u}_{h}^{n+1}\right), \eta_{\mathbf{u}}^{n+1}, \varphi_{\mathbf{u}}^{n+1}\right),
\end{aligned}
$$

and

$$
\begin{aligned}
F_{2}\left(\varphi_{\sigma}^{n+1}\right)= & \lambda\left(\beth\left(\Pi_{\sigma}\left(\sigma^{n+1}\right)\right)-\partial_{t} \sigma^{n+1}, \varphi_{\sigma}^{n+1}\right)+2 \alpha\left(D\left(\eta_{\mathbf{u}}^{n+1}\right), \varphi_{\sigma}^{n+1}\right) \\
& -\left(\eta_{\sigma}^{n+1}, \varphi_{\sigma}^{n+1}\right)-\lambda B\left(\digamma\left(\varphi_{\mathbf{u}}^{n+1}\right), \sigma^{n+1}, \varphi_{\sigma}^{n+1}\right)-\lambda B\left(\digamma\left(\eta_{\mathbf{u}}^{n+1}\right), \sigma^{n+1}, \varphi_{\sigma}^{n+1}\right) \\
& -\lambda B\left(\digamma\left(\mathbf{u}_{h}^{n+1}\right), \eta_{\sigma}^{n+1}, \varphi_{\sigma}^{n+1}\right)+\lambda B\left(\digamma\left(\mathbf{u}^{n+1}\right)-\mathbf{u}^{n+1}, \sigma^{n+1}, \varphi_{\sigma}^{n+1}\right)
\end{aligned}
$$




$$
\begin{aligned}
& -\lambda\left(g_{a}\left(\digamma\left(\varphi_{\sigma}^{n+1}\right), \nabla \mathbf{u}^{n+1}\right), \varphi_{\sigma}^{n+1}\right)-\lambda\left(g_{a}\left(\digamma\left(\eta_{\sigma}^{n+1}\right), \nabla \mathbf{u}^{n+1}\right), \varphi_{\sigma}^{n+1}\right) \\
& -\lambda\left(g_{a}\left(\digamma\left(\sigma_{h}^{n+1}\right), \nabla \varphi_{\mathbf{u}}^{n+1}\right), \varphi_{\sigma}^{n+1}\right)-\lambda\left(g_{a}\left(\digamma\left(\sigma_{h}^{n+1}\right), \nabla \eta_{\mathbf{u}}^{n+1}\right), \varphi_{\sigma}^{n+1}\right) \\
& +\lambda\left(g_{a}\left(\digamma\left(\sigma^{n+1}\right)-\sigma^{n+1}, \nabla \mathbf{u}^{n+1}\right), \varphi_{\sigma}^{n+1}\right) .
\end{aligned}
$$

Multiplying (4.7a) by $2 \alpha$ and adding to (4.7b), using the 'coercivity' $(2.10)$ of $B(\cdot, \cdot, \cdot)$ yield the single equation

$$
\begin{aligned}
2 \alpha \operatorname{Re} & \left(\beth\left(\varphi_{\mathbf{u}}^{n+1}\right), \varphi_{\mathbf{u}}^{n+1}\right)+4 \alpha(1-\alpha)\left\|D\left(\varphi_{\mathbf{u}}^{n+1}\right)\right\|^{2}+\lambda\left(\beth\left(\varphi_{\sigma}^{n+1}\right), \varphi_{\sigma}^{n+1}\right) \\
& +\left\|\varphi_{\sigma}^{n+1}\right\|^{2}+\frac{\lambda}{2}\left\|\varphi_{\sigma}^{n+1,+}-\varphi_{\sigma}^{n+1,-}\right\|_{h, \digamma}^{2}\left(\mathbf{u}_{h}^{n+1}\right) \\
=2 & \alpha F_{1}\left(\varphi_{\mathbf{u}}^{n+1}\right)+F_{2}\left(\varphi_{\sigma}^{n+1}\right) .
\end{aligned}
$$

Applying identity (2.13) to (4.10) yields

$$
\begin{aligned}
\frac{2 \alpha R e}{4 \triangle t} & {\left[3\left\|\varphi_{\mathbf{u}}^{n+1}\right\|^{2}-4\left\|\varphi_{\mathbf{u}}^{n}\right\|^{2}+\left\|\varphi_{\mathbf{u}}^{n-1}\right\|^{2}\right]+\frac{2 \alpha R e}{2 \triangle t}\left[\left\|\varphi_{\mathbf{u}}^{n+1}-\varphi_{\mathbf{u}}^{n}\right\|^{2}\right.} \\
& \left.-\left\|\varphi_{\mathbf{u}}^{n}-\varphi_{\mathbf{u}}^{n-1}\right\|^{2}\right]+\frac{2 \alpha R e}{4 \triangle t}\left[\left\|\varphi_{\mathbf{u}}^{n+1}-2 \varphi_{\mathbf{u}}^{n}+\varphi_{\mathbf{u}}^{n-1}\right\|^{2}\right]+\frac{\lambda}{4 \triangle t}\left[3\left\|\varphi_{\sigma}^{n+1}\right\|^{2}\right. \\
& \left.-4\left\|\varphi_{\sigma}^{n}\right\|^{2}+\left\|\varphi_{\sigma}^{n-1}\right\|^{2}\right]+\frac{\lambda}{2 \triangle t}\left[\left\|\varphi_{\sigma}^{n+1}-\varphi_{\sigma}^{n}\right\|^{2}-\left\|\varphi_{\sigma}^{n}-\varphi_{\sigma}^{n-1}\right\|^{2}\right] \\
& +\frac{\lambda}{4 \triangle t}\left[\left\|\varphi_{\sigma}^{n+1}-2 \varphi_{\sigma}^{n}+\varphi_{\sigma}^{n-1}\right\|^{2}\right]+\left\|\varphi_{\sigma}^{n+1}\right\|^{2}+4 \alpha(1-\alpha)\left\|D\left(\varphi_{\mathbf{u}}^{n+1}\right)\right\|^{2} \\
& +\frac{\lambda}{2}\left\|\varphi_{\sigma}^{n+1,+}-\varphi_{\sigma}^{n+1,-}\right\|_{h, \digamma}^{2}\left(\mathbf{u}_{h}^{n+1}\right) \\
= & 2 \alpha F_{1}\left(\varphi_{\mathbf{u}}^{n+1}\right)+F_{2}\left(\varphi_{\sigma}^{n+1}\right) .
\end{aligned}
$$

Multiplying both sides of (4.11) by $2 \Delta t$, summing (4.11) with respect to $n$ from 0 to $l$ and using identity (3.17) give

$$
\begin{aligned}
& \alpha \operatorname{Re}\left\|\varphi_{\mathbf{u}}^{l+1}\right\|^{2}+\alpha \operatorname{Re}\left\|2 \varphi_{\mathbf{u}}^{l+1}-\varphi_{\mathbf{u}}^{l}\right\|^{2}+\frac{\lambda}{2}\left\|\varphi_{\sigma}^{l+1}\right\|^{2}+\frac{\lambda}{2}\left\|2 \varphi_{\sigma}^{l+1}-\varphi_{\sigma}^{l}\right\|^{2} \\
& +2 \Delta t \sum_{n=0}^{l}\left\|\varphi_{\sigma}^{n+1}\right\|^{2}+8 \alpha(1-\alpha) \Delta t \sum_{n=0}^{l}\left\|D\left(\varphi_{\mathbf{u}}^{n+1}\right)\right\|^{2} \\
& +\lambda \Delta t \sum_{n=0}^{l}\left\|\varphi_{\sigma}^{n+1,+}-\varphi_{\sigma}^{n+1,-}\right\|_{h, \digamma\left(\mathbf{u}_{h}^{n+1}\right)}^{2} \\
& \leq 2 \alpha \operatorname{Re}\left\|\varphi_{\mathbf{u}}^{0}\right\|^{2}+\lambda\left\|\varphi_{\sigma}^{0}\right\|^{2}+2 \Delta t \sum_{n=0}^{l}\left[2 \alpha F_{1}\left(\varphi_{\mathbf{u}}^{n+1}\right)+F_{2}\left(\varphi_{\sigma}^{n+1}\right)\right] .
\end{aligned}
$$

Note that

$$
\begin{aligned}
\left\|\digamma\left(\sigma_{h}^{n+1}\right)\right\|^{2} & =\left\|2 \sigma_{h}^{n}-\sigma_{h}^{n-1}\right\|^{2} \leq 4\left\|\sigma_{h}^{n}\right\|^{2}+\left\|\sigma_{h}^{n-1}\right\|^{2}+4\left\|\sigma_{h}^{n}\right\|\left\|\sigma_{h}^{n-1}\right\| \\
& \leq 4\left\|\sigma_{h}^{n}\right\|^{2}+\left\|\sigma_{h}^{n-1}\right\|^{2}+4\left[\frac{1}{4}\left\|\sigma_{h}^{n}\right\|^{2}+\left\|\sigma_{h}^{n-1}\right\|^{2}\right] \\
& =5\left\|\sigma_{h}^{n}\right\|^{2}+5\left\|\sigma_{h}^{n-1}\right\|^{2}
\end{aligned}
$$


and

$$
\left\|\digamma\left(\mathbf{u}_{h}^{n+1}\right)\right\|=\left\|2 \mathbf{u}_{h}^{n}-\mathbf{u}_{h}^{n-1}\right\| \leq 2\left\|\mathbf{u}_{h}^{n}\right\|+\left\|\mathbf{u}_{h}^{n-1}\right\| .
$$

We proceed to bound each term on the RHS of (4.12), absorb like-terms into the lefthand side.

We first estimate the linear terms of $F_{1}\left(\varphi_{\mathbf{u}}^{n+1}\right)$ in (4.12). For the pressure term, using the Cauchy-Schwarz, Korn's and Young's inequalities, we have

$$
\begin{aligned}
\left|\left(p^{n+1}, \nabla \cdot \varphi_{\mathbf{u}}^{n+1}\right)\right| & =\left|\left(p^{n+1}-\Pi_{p}\left(p^{n+1}\right), \nabla \cdot \varphi_{\mathbf{u}}^{n+1}\right)\right| \\
& \leq \sqrt{d}\left\|p^{n+1}-\Pi_{p}\left(p^{n+1}\right)\right\|\left\|\nabla \varphi_{\mathbf{u}}^{n+1}\right\| \\
& \leq \sqrt{d} C_{k}\left\|p^{n+1}-\Pi_{p}\left(p^{n+1}\right)\right\|\left\|D\left(\varphi_{\mathbf{u}}^{n+1}\right)\right\| \\
& \leq \epsilon_{2}\left\|D\left(\varphi_{\mathbf{u}}^{n+1}\right)\right\|^{2}+\frac{d C_{k}^{2}}{4 \epsilon_{2}}\left\|p^{n+1}-\Pi_{p}\left(p^{n+1}\right)\right\|^{2} .
\end{aligned}
$$

Similarly, we see that

$$
\begin{aligned}
& \left(\eta_{\sigma}^{n+1}, D\left(\varphi_{\mathbf{u}}^{n+1}\right)\right) \leq \epsilon_{3}\left\|D\left(\varphi_{\mathbf{u}}^{n+1}\right)\right\|^{2}+\frac{1}{4 \epsilon_{3}}\left\|\eta_{\sigma}^{n+1}\right\|^{2}, \\
& 2(1-\alpha)\left(D\left(\eta_{\mathbf{u}}^{n+1}\right), D\left(\varphi_{\mathbf{u}}^{n+1}\right)\right) \leq \epsilon_{4}\left\|D\left(\varphi_{\mathbf{u}}^{n+1}\right)\right\|^{2}+\frac{(1-\alpha)^{2}}{\epsilon_{4}}\left\|D\left(\eta_{\mathbf{u}}^{n+1}\right)\right\|^{2} \\
& \operatorname{Re}\left(\beth\left(\Pi_{\mathbf{u}}\left(\mathbf{u}^{n+1}\right)\right)-\partial_{t} \mathbf{u}^{n+1}, \varphi_{\mathbf{u}}^{n+1}\right) \\
& \quad \leq \operatorname{Re}\left\|\beth\left(\Pi_{\mathbf{u}}\left(\mathbf{u}^{n+1}\right)\right)-\partial_{t} \mathbf{u}^{n+1}\right\|\left\|\varphi_{\mathbf{u}}^{n+1}\right\| \\
& \left.\quad \leq \epsilon_{5} \operatorname{Re} \| \varphi_{\mathbf{u}}^{n+1}\right)\left\|^{2}+\frac{\operatorname{Re}}{4 \epsilon_{5}}\right\| \beth\left(\Pi_{\mathbf{u}}\left(\mathbf{u}^{n+1}\right)\right)-\partial_{t} \mathbf{u}^{n+1} \|^{2} .
\end{aligned}
$$

For the nonlinear terms of $F_{1}\left(\varphi_{\mathbf{u}}^{n+1}\right)$, using the Cauchy-Schwarz inequality, Young's inequality, the regularity assumption (2.3) of velocity and hypothesis IH1, we obtain

$$
\begin{aligned}
& \operatorname{Rec}\left(\digamma\left(\mathbf{u}^{n+1}\right)-\mathbf{u}^{n+1}, \mathbf{u}^{n+1}, \varphi_{\mathbf{u}}^{n+1}\right) \\
& \leq d \operatorname{Re}\left\|\digamma\left(\mathbf{u}^{n+1}\right)-\mathbf{u}^{n+1}\right\|\left\|\nabla \mathbf{u}^{n+1}\right\|_{\infty}\left\|\varphi_{\mathbf{u}}^{n+1}\right\| \\
& \leq d \operatorname{MRe}\left\|\digamma\left(\mathbf{u}^{n+1}\right)-\mathbf{u}^{n+1}\right\|\left\|\varphi_{\mathbf{u}}^{n+1}\right\| \\
& \leq \epsilon_{6} \operatorname{Re}\left\|\varphi_{\mathbf{u}}^{n+1}\right\|^{2}+\frac{d^{2} M^{2} \operatorname{Re}\left\|\digamma\left(\mathbf{u}^{n+1}\right)-\mathbf{u}^{n+1}\right\|^{2},}{4 \epsilon_{6}} \| \\
& \operatorname{Rec}\left(\digamma\left(\varphi_{\mathbf{u}}^{n+1}\right), \mathbf{u}^{n+1}, \varphi_{\mathbf{u}}^{n+1}\right) \\
& \leq \operatorname{Red}\left\|\digamma\left(\varphi_{\mathbf{u}}^{n+1}\right)\right\|\left\|\nabla \mathbf{u}^{n+1}\right\| \varphi_{\infty}\left\|\varphi_{\mathbf{u}}^{n+1}\right\| \\
& \leq \epsilon_{7} \operatorname{Re}\left\|\varphi_{\mathbf{u}}^{n+1}\right\|^{2}+\frac{\operatorname{Red}^{2} M^{2}}{4 \epsilon_{7}}\left\|\digamma\left(\varphi_{\mathbf{u}}^{n+1}\right)\right\|^{2} \\
& \leq \epsilon_{7} \operatorname{Re}\left\|\varphi_{\mathbf{u}}^{n+1}\right\|^{2}+\frac{\operatorname{Red}^{2} M^{2}}{4 \epsilon_{7}}\left\|2 \varphi_{\mathbf{u}}^{n}-\varphi_{\mathbf{u}}^{n-1}\right\|^{2},
\end{aligned}
$$




$$
\begin{aligned}
& \operatorname{Rec}\left(\digamma\left(\eta_{\mathbf{u}}^{n+1}\right), \mathbf{u}^{n+1}, \varphi_{\mathbf{u}}^{n+1}\right) \\
& \leq \operatorname{Red}\left\|\digamma\left(\eta_{\mathbf{u}}^{n+1}\right)\right\|\left\|\nabla \mathbf{u}^{n+1}\right\|_{\infty}\left\|\varphi_{\mathbf{u}}^{n+1}\right\| \\
& \leq \epsilon_{8} \operatorname{Re}\left\|\varphi_{\mathbf{u}}^{n+1}\right\|^{2}+\frac{\operatorname{Red}^{2} M^{2}}{4 \epsilon_{8}}\left\|\digamma\left(\eta_{\mathbf{u}}^{n+1}\right)\right\|^{2} \\
& \operatorname{Rec}\left(\digamma\left(\mathbf{u}_{h}^{n+1}\right), \eta_{\mathbf{u}}^{n+1}, \varphi_{\mathbf{u}}^{n+1}\right) \leq \operatorname{Red}\left\|\digamma\left(\mathbf{u}_{h}^{n+1}\right)\right\|_{\infty}\left\|\nabla \eta_{\mathbf{u}}^{n+1}\right\|\left\|\varphi_{\mathbf{u}}^{n+1}\right\| \\
& \leq 3 \operatorname{RedK} C_{k}\left\|D\left(\eta_{\mathbf{u}}^{n+1}\right)\right\|\left\|\varphi_{\mathbf{u}}^{n+1}\right\| \\
& \leq \epsilon_{9} \operatorname{Re}\left\|\varphi_{\mathbf{u}}^{n+1}\right\|^{2}+\frac{9 \operatorname{Red}^{2} K^{2} C_{k}^{2}}{4 \epsilon_{9}}\left\|D\left(\eta_{\mathbf{u}}^{n+1}\right)\right\|^{2} .
\end{aligned}
$$

Combining (4.15)-(4.22), we have the following estimate of $2 \alpha F_{1}\left(\varphi_{\mathbf{u}}^{n+1}\right)$ :

$$
\begin{aligned}
2 \alpha F_{1}\left(\varphi_{\mathbf{u}}^{n+1}\right) \leq & 2 \alpha \operatorname{Re}\left(\epsilon_{5}+\epsilon_{6}+\epsilon_{7}+\epsilon_{8}+\epsilon_{9}\right)\left\|\varphi_{\mathbf{u}}^{n+1}\right\|^{2} \\
& +\frac{\alpha d^{2} M^{2} R e}{2 \epsilon_{7}}\left\|2 \varphi_{\mathbf{u}}^{n}-\varphi_{\mathbf{u}}^{n-1}\right\|^{2}+2 \alpha\left(\epsilon_{2}+\epsilon_{3}+\epsilon_{4}\right)\left\|D\left(\varphi_{\mathbf{u}}^{n+1}\right)\right\|^{2} \\
& +\frac{d C_{k}^{2} \alpha}{2 \epsilon_{2}}\left\|p^{n+1}-\Pi_{p}\left(p^{n+1}\right)\right\|^{2}+\frac{\alpha}{2 \epsilon_{3}}\left\|\eta_{\sigma}^{n+1}\right\|^{2}+\frac{2 \alpha(1-\alpha)^{2}}{\epsilon_{4}}\left\|D\left(\eta_{\mathbf{u}}^{n+1}\right)\right\|^{2} \\
& +\frac{\operatorname{Re} \alpha}{2 \epsilon_{5}}\left\|\beth\left(\Pi_{\mathbf{u}}\left(\mathbf{u}^{n+1}\right)\right)-\partial_{t} \mathbf{u}^{n+1}\right\|^{2}+\frac{d^{2} \alpha M^{2} \operatorname{Re}}{2 \epsilon_{6}}\left\|\digamma\left(\mathbf{u}^{n+1}\right)-\mathbf{u}^{n+1}\right\|^{2} \\
& +\frac{\alpha \operatorname{Red} M^{2}}{2 \epsilon_{8}}\left\|\digamma\left(\eta_{\mathbf{u}}^{n+1}\right)\right\|^{2}
\end{aligned}
$$

Now we bound the terms of $F_{2}\left(\varphi_{\sigma}^{n+1}\right)$ in (4.12). For the first three linear terms, applying the Cauchy-Schwarz inequality and Young's inequality, we obtain

$$
\begin{gathered}
\lambda\left(\beth\left(\Pi_{\sigma}\left(\sigma^{n+1}\right)\right)-\partial_{t} \sigma^{n+1}, \varphi_{\sigma}^{n+1}\right) \\
\leq \lambda\left\|\beth\left(\Pi_{\sigma}\left(\sigma^{n+1}\right)\right)-\partial_{t} \sigma^{n+1}\right\|\left\|\varphi_{\sigma}^{n+1}\right\| \\
\leq \beta_{1} \lambda\left\|\varphi_{\sigma}^{n+1}\right\|^{2}+\frac{\lambda}{4 \beta_{1}}\left\|\beth\left(\Pi_{\sigma}\left(\sigma^{n+1}\right)\right)-\partial_{t} \sigma^{n+1}\right\|^{2}, \\
\left(\eta_{\sigma}^{n+1}, \varphi_{\sigma}^{n+1}\right) \leq\left\|\eta_{\sigma}^{n+1}\right\|\left\|\varphi_{\sigma}^{n+1}\right\| \leq \beta_{2}\left\|\varphi_{\sigma}^{n+1}\right\|^{2}+\frac{1}{4 \beta_{2}}\left\|\eta_{\sigma}^{n+1}\right\|^{2}, \\
2 \alpha\left(D\left(\eta_{\mathbf{u}}^{n+1}\right), \varphi_{\sigma}^{n+1}\right) \leq 2 \alpha\left\|D\left(\eta_{\mathbf{u}}^{n+1}\right)\right\|\left\|\varphi_{\sigma}^{n+1}\right\| \\
\leq \beta_{3}\left\|\varphi_{\sigma}^{n+1}\right\|^{2}+\frac{\alpha^{2}}{\beta_{3}}\left\|D\left(\eta_{\mathbf{u}}^{n+1}\right)\right\|^{2} .
\end{gathered}
$$

We estimate some nonlinear terms of the convection term about $\sigma$. The first nonlinear term $B\left(\digamma\left(\varphi_{\mathbf{u}}^{n+1}\right), \sigma^{n+1}, \varphi_{\sigma}^{n+1}\right)$ of $F_{2}\left(\varphi_{\sigma}^{n+1}\right)$ can be rewritten as

$$
\begin{aligned}
\lambda B( & \left.\digamma\left(\varphi_{\mathbf{u}}^{n+1}\right), \sigma^{n+1}, \varphi_{\sigma}^{n+1}\right) \\
= & \lambda\left(\digamma\left(\varphi_{\mathbf{u}}^{n+1}\right) \cdot \nabla \sigma^{n+1}, \varphi_{\sigma}^{n+1}\right)_{h} \\
& +\frac{\lambda}{2}\left(\left(\nabla \cdot \digamma\left(\varphi_{\mathbf{u}}^{n+1}\right)\right) \sigma^{n+1}, \varphi_{\sigma}^{n+1}\right)+\lambda\left\langle\sigma^{n+1,+}-\sigma^{n+1,-}, \varphi_{\sigma}^{n+1,+}\right\rangle_{h, \digamma\left(\varphi_{\mathbf{u}}^{n+1}\right)}
\end{aligned}
$$


Note that the term $\lambda\left\langle\sigma^{n+1,+}-\sigma^{n+1,-}, \varphi_{\sigma}^{n+1,+}\right\rangle_{h, \digamma\left(\varphi_{\mathbf{u}}^{n+1}\right)}=0$ due to the continuity of $\sigma$. The other two terms on the RHS of (4.27) may be bounded by

$$
\begin{aligned}
\lambda( & \left.\left(\varphi_{\mathbf{u}}^{n+1}\right) \cdot \nabla \sigma^{n+1}, \varphi_{\sigma}^{n+1}\right)_{h} \\
& \leq \lambda\left\|\digamma\left(\varphi_{\mathbf{u}}^{n+1}\right) \cdot \nabla \sigma^{n+1}\right\|\left\|\varphi_{\sigma}^{n+1}\right\| \\
& \leq \lambda \sqrt{d^{3}}\left\|\nabla \sigma^{n+1}\right\|_{\infty}\left\|\digamma\left(\varphi_{\mathbf{u}}^{n+1}\right)\right\|\left\|\varphi_{\sigma}^{n+1}\right\| \leq \lambda \sqrt{d^{3}} M\left\|\digamma\left(\varphi_{\mathbf{u}}^{n+1}\right)\right\|\left\|\varphi_{\sigma}^{n+1}\right\| \\
& \leq \beta_{4} \lambda\left\|\varphi_{\sigma}^{n+1}\right\|^{2}+\frac{\lambda d^{3} M^{2}}{4 \beta_{4}}\left\|2 \varphi_{\mathbf{u}}^{n}-\varphi_{\mathbf{u}}^{n-1}\right\|^{2} \\
\frac{\lambda}{2}( & \left.\left(\nabla \cdot \digamma\left(\varphi_{\mathbf{u}}^{n+1}\right)\right) \sigma^{n+1}, \varphi_{\sigma}^{n+1}\right) \\
& \leq \frac{d \lambda}{2}\left\|\nabla \cdot \digamma\left(\varphi_{\mathbf{u}}^{n+1}\right)\right\|\left\|\sigma^{n+1}\right\| \varphi_{\infty}\left\|\varphi_{\sigma}^{n+1}\right\| \\
& \leq \frac{\sqrt{d^{3}} M \lambda}{2}\left\|\nabla \digamma\left(\varphi_{\mathbf{u}}^{n+1}\right)\right\|\left\|\varphi_{\sigma}^{n+1}\right\| \\
& \leq \frac{\sqrt{d^{3}} M C_{k} \lambda}{2}\left\|D\left(\digamma\left(\varphi_{\mathbf{u}}^{n+1}\right)\right)\right\|\left\|\varphi_{\sigma}^{n+1}\right\| \\
& \leq \frac{2 \alpha \epsilon_{10}}{10}\left\|D\left(\digamma\left(\varphi_{\mathbf{u}}^{n+1}\right)\right)\right\|^{2}+\frac{10 d^{3} C_{k}^{2} M^{2} \lambda^{2}}{32 \epsilon_{10} \alpha}\left\|\varphi_{\sigma}^{n+1}\right\|^{2} \\
& \leq \alpha \epsilon_{10}\left(\left\|D\left(\varphi_{\mathbf{u}}^{n}\right)\right\|^{2}+\left\|D\left(\varphi_{\mathbf{u}}^{n-1}\right)\right\|^{2}\right)+\frac{10 d^{3} C_{k}^{2} M^{2} \lambda^{2}}{32 \epsilon_{10} \alpha}\left\|\varphi_{\sigma}^{n+1}\right\|^{2}
\end{aligned}
$$

Similarly as (4.27), we write the second nonlinear term $\lambda B\left(\digamma\left(\eta_{\mathbf{u}}^{n+1}\right), \sigma^{n+1}, \varphi_{\sigma}^{n+1}\right)$ of $F_{2}\left(\varphi_{\sigma}^{n+1}\right)$ as

$$
\begin{aligned}
\lambda B( & \left.\digamma\left(\eta_{\mathbf{u}}^{n+1}\right), \sigma^{n+1}, \varphi_{\sigma}^{n+1}\right) \\
= & \lambda\left(\digamma\left(\eta_{\mathbf{u}}^{n+1}\right) \cdot \nabla \sigma^{n+1}, \varphi_{\sigma}^{n+1}\right)_{h} \\
& +\frac{\lambda}{2}\left(\left(\nabla \cdot \digamma\left(\eta_{\mathbf{u}}^{n+1}\right)\right) \sigma^{n+1}, \varphi_{\sigma}^{n+1}\right)+\lambda\left\langle\sigma^{n+1,+}-\sigma^{n+1,-}, \varphi_{\sigma}^{n+1,+}\right\rangle_{h, \digamma\left(\eta_{\mathbf{u}}^{n+1}\right)^{.}}
\end{aligned}
$$

Using the same method as (4.27) to estimate the three terms on the RHS of (4.30) leads to

$$
\begin{aligned}
& \lambda\left(\digamma\left(\eta_{\mathbf{u}}^{n+1}\right) \cdot \nabla \sigma^{n+1}, \varphi_{\sigma}^{n+1}\right)_{h} \\
& \quad \leq \lambda\left\|\digamma\left(\eta_{\mathbf{u}}^{n+1}\right) \cdot \nabla \sigma^{n+1}\right\|\left\|\varphi_{\sigma}^{n+1}\right\| \\
& \quad \leq \lambda \sqrt{d^{3}}\left\|\digamma\left(\eta_{\mathbf{u}}^{n+1}\right)\right\|\left\|\nabla \sigma^{n+1}\right\|_{\infty}\left\|\varphi_{\sigma}^{n+1}\right\| \\
& \quad \leq \lambda \sqrt{d^{3}} M\left\|\digamma\left(\eta_{\mathbf{u}}^{n+1}\right)\right\|\left\|\varphi_{\sigma}^{n+1}\right\| \\
& \quad \leq \beta_{5} \lambda\left\|\varphi_{\sigma}^{n+1}\right\|^{2}+\frac{\lambda d^{3} M^{2}}{4 \beta_{5}}\left\|\digamma\left(\eta_{\mathbf{u}}^{n+1}\right)\right\|^{2} \\
& \frac{\lambda}{2}\left(\left(\nabla \cdot \digamma\left(\eta_{\mathbf{u}}^{n+1}\right)\right) \sigma^{n+1}, \varphi_{\sigma}^{n+1}\right) \\
& \quad \leq \frac{\lambda d}{2}\left\|\nabla \cdot \digamma\left(\eta_{\mathbf{u}}^{n+1}\right)\right\|\left\|\sigma^{n+1}\right\|_{\infty}\left\|\varphi_{\sigma}^{n+1}\right\|
\end{aligned}
$$




$$
\begin{gathered}
\leq \frac{\lambda \sqrt{d^{3}} M}{2}\left\|\nabla \digamma\left(\eta_{\mathbf{u}}^{n+1}\right)\right\|\left\|\varphi_{\sigma}^{n+1}\right\| \\
\leq \beta_{6} \lambda\left\|\varphi_{\sigma}^{n+1}\right\|^{2}+\frac{\lambda d^{3} M^{2}}{16 \beta_{6}}\left\|\nabla \digamma\left(\eta_{\mathbf{u}}^{n+1}\right)\right\|^{2}, \\
\lambda\left\langle\sigma^{n+1,+}-\sigma^{n+1,-}, \varphi_{\sigma}^{n+1,+}\right\rangle_{h, \digamma\left(\eta_{\mathbf{u}}^{n+1}\right)}=0,
\end{gathered}
$$

where we have used the continuity of $\sigma^{n+1}$ in (4.33).

By the same way, the third nonlinear term $\lambda B\left(\digamma\left(\mathbf{u}_{h}^{n+1}\right), \eta_{\sigma}^{n+1}, \varphi_{\sigma}^{n+1}\right)$ of $F_{2}\left(\varphi_{\sigma}^{n+1}\right)$ can be written as

$$
\begin{aligned}
\lambda B( & \left.\digamma\left(\mathbf{u}_{h}^{n+1}\right), \eta_{\sigma}^{n+1}, \varphi_{\sigma}^{n+1}\right) \\
= & \lambda\left(\digamma\left(\mathbf{u}_{h}^{n+1}\right) \cdot \nabla \eta_{\sigma}^{n+1}, \varphi_{\sigma}^{n+1}\right)_{h} \\
& +\frac{\lambda}{2}\left(\nabla \cdot \digamma\left(\mathbf{u}_{h}^{n+1}\right) \eta_{\sigma}^{n+1}, \varphi_{\sigma}^{n+1}\right)+\lambda\left\langle\eta_{\sigma}^{n+1,+}-\eta_{\sigma}^{n+1,-}, \varphi_{\sigma}^{n+1,+}\right\rangle_{h, \digamma\left(\mathbf{u}_{h}^{n+1}\right)} .
\end{aligned}
$$

For the first term in (4.34), using (4.14) and hypothesis IH1, we can get

$$
\begin{aligned}
& \lambda\left(\digamma\left(\mathbf{u}_{h}^{n+1}\right) \cdot \nabla \eta_{\sigma}^{n+1}, \varphi_{\sigma}^{n+1}\right)_{h} \\
& \quad \leq \lambda\left\|\digamma\left(\mathbf{u}_{h}^{n+1}\right) \cdot \nabla \eta_{\sigma}^{n+1}\right\|\left\|\varphi_{\sigma}^{n+1}\right\| \\
& \quad \leq \sqrt{d} \lambda\left\|\digamma\left(\mathbf{u}_{h}^{n+1}\right)\right\|_{\infty}\left\|\nabla \eta_{\sigma}^{n+1}\right\|\left\|\varphi_{\sigma}^{n+1}\right\| \\
& \quad \leq 3 \sqrt{d} \lambda K\left\|\nabla \eta_{\sigma}^{n+1}\right\|\left\|\varphi_{\sigma}^{n+1}\right\| \\
& \quad \leq \beta_{7} \lambda\left\|\varphi_{\sigma}^{n+1}\right\|^{2}+\frac{9 \lambda d K^{2}}{4 \beta_{7}}\left\|\nabla \eta_{\sigma}^{n+1}\right\|^{2} .
\end{aligned}
$$

Making use of inverse inequality (2.8), (4.14) and hypothesis IH1 to the second term in (4.34) yields

$$
\begin{aligned}
\frac{\lambda}{2}\left(\nabla \cdot \digamma\left(\mathbf{u}_{h}^{n+1}\right) \eta_{\sigma}^{n+1}, \varphi_{\sigma}^{n+1}\right) & \leq \frac{\lambda}{2} d\left\|\nabla \digamma\left(\mathbf{u}_{h}^{n+1}\right)\right\|_{\infty}\left\|\eta_{\sigma}^{n+1}\right\|\left\|\varphi_{\sigma}^{n+1}\right\| \\
& \leq \frac{\lambda d}{2} C_{i} h^{-1}\left\|\digamma\left(\mathbf{u}_{h}^{n+1}\right)\right\|_{\infty}\left\|\eta_{\sigma}^{n+1}\right\|\left\|\varphi_{\sigma}^{n+1}\right\| \\
& \leq \beta_{8} \lambda\left\|\varphi_{\sigma}^{n+1}\right\|^{2}+\frac{9 \lambda d^{2} K^{2} C_{i}^{2} h^{-2}}{16 \beta_{8}}\left\|\eta_{\sigma}^{n+1}\right\|^{2} .
\end{aligned}
$$

Applying the continuity of $\eta_{\sigma}^{n+1}$ to the third term on the RHS of (4.34) leads to

$$
\lambda\left\langle\eta_{\sigma}^{n+1,+}-\eta_{\sigma}^{n+1,-}, \varphi_{\sigma}^{n+1,+}\right\rangle_{h, \digamma\left(\mathbf{u}_{h}^{n+1}\right)}=0
$$

Using $\nabla \cdot \mathbf{u}=0$ and the continuity of $\sigma^{n+1}$ to the term $\lambda B\left(\digamma\left(\mathbf{u}^{n+1}\right)-\mathbf{u}^{n+1}, \sigma^{n+1}, \varphi_{\sigma}^{n+1}\right)$, we obtain

$$
\begin{aligned}
& \lambda \mid B(\left.\digamma\left(\mathbf{u}^{n+1}\right)-\mathbf{u}^{n+1}, \sigma^{n+1}, \varphi_{\sigma}^{n+1}\right) \mid \\
& \leq \lambda\left|\left(\left(\digamma\left(\mathbf{u}^{n+1}\right)-\mathbf{u}^{n+1}\right) \cdot \nabla \sigma^{n+1}, \varphi_{\sigma}^{n+1}\right)_{h}\right| \\
& \quad+\frac{\lambda}{2} \mid\left(\left(\nabla \cdot\left(\digamma\left(\mathbf{u}^{n+1}\right)-\mathbf{u}^{n+1}\right) \sigma^{n+1}, \varphi_{\sigma}^{n+1}\right) \mid\right.
\end{aligned}
$$




$$
\begin{aligned}
& +\lambda|| \sigma^{n+1,+}-\sigma^{n+1,-},\left.\varphi_{\sigma}^{n+1,+}\right|_{h, \digamma\left(\mathbf{u}^{n+1}\right)-\mathbf{u}^{n+1}} \mid \\
\leq & \lambda\left\|\left(\digamma\left(\mathbf{u}^{n+1}\right)-\mathbf{u}^{n+1}\right) \cdot \nabla \sigma^{n+1}\right\|\left\|\varphi_{\sigma}^{n+1}\right\| \\
\leq & \lambda \sqrt{d^{3}}\left\|\nabla \sigma^{n+1}\right\|_{\infty}\left\|\digamma\left(\mathbf{u}^{n+1}\right)-\mathbf{u}^{n+1}\right\|\left\|\varphi_{\sigma}^{n+1}\right\| \\
\leq & \beta_{9} \lambda\left\|\varphi_{\sigma}^{n+1}\right\|^{2}+\frac{\lambda d^{3} M^{2}}{4 \beta_{9}}\left\|\digamma\left(\mathbf{u}^{n+1}\right)-\mathbf{u}^{n+1}\right\|^{2} .
\end{aligned}
$$

We will estimate the last five terms of $F_{2}\left(\varphi_{\sigma}^{n+1}\right)$ in (4.12). Applying the Cauchy-Schwarz inequality, Young's inequality, Korn's inequality, the regularity assumption (2.3) of velocity and hypothesis IH1, we can obtain

$$
\begin{aligned}
& \lambda\left(g_{a}\left(\digamma\left(\varphi_{\sigma}^{n+1}\right), \nabla \mathbf{u}^{n+1}\right), \varphi_{\sigma}^{n+1}\right) \leq \lambda\left\|g_{a}\left(\digamma\left(\varphi_{\sigma}^{n+1}\right), \nabla \mathbf{u}^{n+1}\right)\right\|\left\|\varphi_{\sigma}^{n+1}\right\| \\
& \leq 4 \lambda d\left\|\digamma\left(\varphi_{\sigma}^{n+1}\right)\right\|\left\|\nabla \mathbf{u}^{n+1}\right\|_{\infty}\left\|\varphi_{\sigma}^{n+1}\right\| \\
& \leq 4 \lambda d M\left\|\digamma\left(\varphi_{\sigma}^{n+1}\right)\right\|\left\|\varphi_{\sigma}^{n+1}\right\| \\
& \leq \beta_{10} \lambda\left\|\varphi_{\sigma}^{n+1}\right\|^{2}+\frac{4 \lambda d^{2} M^{2}}{\beta_{10}}\left\|2 \varphi_{\sigma}^{n}-\varphi_{\sigma}^{n-1}\right\|^{2}, \\
& \lambda\left(g_{a}\left(\digamma\left(\eta_{\sigma}^{n+1}\right), \nabla \mathbf{u}^{n+1}\right), \varphi_{\sigma}^{n+1}\right) \leq \lambda\left\|g_{a}\left(\digamma\left(\eta_{\sigma}^{n+1}\right), \nabla \mathbf{u}^{n+1}\right)\right\|\left\|\varphi_{\sigma}^{n+1}\right\| \\
& \leq 4 \lambda d\left\|\digamma\left(\eta_{\sigma}^{n+1}\right)\right\|\left\|\nabla \mathbf{u}^{n+1}\right\|_{\infty}\left\|\varphi_{\sigma}^{n+1}\right\| \\
& \leq 4 \lambda d M\left\|\digamma\left(\eta_{\sigma}^{n+1}\right)\right\|\left\|\varphi_{\sigma}^{n+1}\right\| \\
& \leq \beta_{11} \lambda\left\|\varphi_{\sigma}^{n+1}\right\|^{2}+\frac{4 \lambda d^{2} M^{2}}{\beta_{11}}\left\|\digamma\left(\eta_{\sigma}^{n+1}\right)\right\|^{2}, \\
& \lambda\left(g_{a}\left(\digamma\left(\sigma_{h}^{n+1}\right), \nabla \varphi_{\mathbf{u}}^{n+1}\right), \varphi_{\sigma}^{n+1}\right) \leq \lambda\left\|g_{a}\left(\digamma\left(\sigma_{h}^{n+1}\right), \nabla \varphi_{\mathbf{u}}^{n+1}\right)\right\|\left\|\varphi_{\sigma}^{n+1}\right\| \\
& \leq 4 d \lambda\left\|\digamma\left(\sigma_{h}^{n+1}\right)\right\|_{\infty}\left\|\nabla \varphi_{\mathbf{u}}^{n+1}\right\|\left\|\varphi_{\sigma}^{n+1}\right\| \\
& \leq 12 d \lambda K C_{k}\left\|D\left(\varphi_{\mathbf{u}}^{n+1}\right)\right\|\left\|\varphi_{\sigma}^{n+1}\right\| \\
& \leq 2 \alpha \epsilon_{11}\left\|D\left(\varphi_{\mathbf{u}}^{n+1}\right)\right\|^{2}+\frac{18 d^{2} \lambda^{2} K^{2} C_{k}^{2}}{\epsilon_{11} \alpha}\left\|\varphi_{\sigma}^{n+1}\right\|^{2}, \\
& \lambda\left(g_{a}\left(\digamma\left(\sigma_{h}^{n+1}\right), \nabla \eta_{\mathbf{u}}^{n+1}\right), \varphi_{\sigma}^{n+1}\right) \leq \lambda\left\|g_{a}\left(\digamma\left(\sigma_{h}^{n+1}\right), \nabla \eta_{\mathbf{u}}^{n+1}\right)\right\|\left\|\varphi_{\sigma}^{n+1}\right\| \\
& \leq 4 d \lambda\left\|\digamma\left(\sigma_{h}^{n+1}\right)\right\|_{\infty}\left\|\nabla \eta_{\mathbf{u}}^{n+1}\right\|\left\|\varphi_{\sigma}^{n+1}\right\| \\
& \leq 12 d \lambda K C_{k}\left\|D\left(\eta_{\mathbf{u}}^{n+1}\right)\right\|\left\|\varphi_{\sigma}^{n+1}\right\| \\
& \leq \beta_{12} \lambda\left\|\varphi_{\sigma}^{n+1}\right\|^{2}+\frac{36 d^{2} \lambda K^{2} C_{k}^{2}}{\beta_{12}}\left\|D\left(\eta_{\mathbf{u}}^{n+1}\right)\right\|^{2}, \\
& \lambda\left(g_{a}\left(\digamma\left(\sigma^{n+1}\right)-\sigma^{n+1}, \nabla \mathbf{u}^{n+1}\right), \varphi_{\sigma}^{n+1}\right) \\
& \leq \lambda\left\|g_{a}\left(\digamma\left(\sigma^{n+1}\right)-\sigma^{n+1}, \nabla \mathbf{u}^{n+1}\right)\right\|\left\|\varphi_{\sigma}^{n+1}\right\| \\
& \leq 4 \lambda d\left\|\digamma\left(\sigma^{n+1}\right)-\sigma^{n+1}\right\|\left\|\nabla \mathbf{u}^{n+1}\right\|_{\infty}\left\|\varphi_{\sigma}^{n+1}\right\| \\
& \leq 4 \lambda d M\left\|\digamma\left(\sigma^{n+1}\right)-\sigma^{n+1}\right\|\left\|\varphi_{\sigma}^{n+1}\right\| \\
& \leq \beta_{13} \lambda\left\|\varphi_{\sigma}^{n+1}\right\|^{2}+\frac{4 d^{2} M^{2} \lambda}{\beta_{13}}\left\|\digamma\left(\sigma^{n+1}\right)-\sigma^{n+1}\right\|^{2} .
\end{aligned}
$$


Combining inequalities (4.24)-(4.43), we obtain the estimate for $F_{2}\left(\varphi_{\sigma}^{n+1}\right)$

$$
\begin{aligned}
F_{2}\left(\varphi_{\sigma}^{n+1}\right) \leq & \left\|\varphi_{\sigma}^{n+1}\right\|^{2} \lambda\left[\beta_{1}+\beta_{4}+\beta_{5}+\cdots+\beta_{13}+\frac{5 d^{3} C_{k}^{2} M^{2} \lambda}{16 \epsilon_{10} \alpha}\right. \\
& \left.+\frac{\left.18 d^{2} \lambda K^{2} C_{k}^{2}\right]}{\epsilon_{11} \alpha}\right]+\left(\beta_{2}+\beta_{3}\right)\left\|\varphi_{\sigma}^{n+1}\right\|^{2}+\frac{4 \lambda^{2} d^{2} M^{2}}{\beta_{10}}\left\|2 \varphi_{\sigma}^{n}-\varphi_{\sigma}^{n-1}\right\|^{2} \\
& +\alpha \epsilon_{10}\left(\left\|D\left(\varphi_{\mathbf{u}}^{n}\right)\right\|^{2}+\left\|D\left(\varphi_{\mathbf{u}}^{n-1}\right)\right\|^{2}\right)+2 \alpha \epsilon_{11}\left\|D\left(\varphi_{\mathbf{u}}^{n+1}\right)\right\|^{2} \\
& +\frac{\lambda d^{3} M^{2}}{4 \beta_{4}}\left\|2 \varphi_{\mathbf{u}}^{n}-\varphi_{\mathbf{u}}^{n-1}\right\|^{2}+\frac{\lambda}{4 \beta_{1}}\left\|\beth\left(\Pi_{\sigma}\left(\sigma^{n+1}\right)\right)-\partial_{t} \sigma^{n+1}\right\|^{2}+\frac{1}{4 \beta_{2}}\left\|\eta_{\sigma}^{n+1}\right\|^{2} \\
& +\frac{\alpha^{2}}{\beta_{3}}\left\|D\left(\eta_{\mathbf{u}}^{n+1}\right)\right\|^{2}+\frac{\lambda d^{3} M^{2}}{4 \beta_{5}}\left\|\digamma\left(\eta_{\mathbf{u}}^{n+1}\right)\right\|^{2}+\frac{\lambda d^{3} M^{2}}{16 \beta_{6}}\left\|\nabla \digamma\left(\eta_{\mathbf{u}}^{n+1}\right)\right\|^{2} \\
& +\frac{9 \lambda d K^{2}}{4 \beta_{7}}\left\|\nabla \eta_{\sigma}^{n+1}\right\|^{2}+\frac{9 \lambda d^{2} K^{2} C_{i}^{2} h^{-2}}{16 \beta_{8}}\left\|\eta_{\sigma}^{n+1}\right\|^{2} \\
& +\frac{\lambda d^{3} M^{2}}{4 \beta_{9}}\left\|\digamma\left(\mathbf{u}^{n+1}\right)-\mathbf{u}^{n+1}\right\|^{2}+\frac{4 \lambda d^{2} M^{2}}{\beta_{11}}\left\|\digamma\left(\eta_{\sigma}^{n+1}\right)\right\|^{2} \\
& +\frac{36 d^{2} \lambda K^{2} C_{k}^{2}}{\beta_{12}}\left\|D\left(\eta_{\mathbf{u}}^{n+1}\right)\right\|^{2}
\end{aligned}
$$

Plugging estimate (4.23) of $2 \alpha F_{1}\left(\varphi_{\mathbf{u}}^{n+1}\right)$ and estimate (4.44) of $F_{2}\left(\varphi_{\sigma}^{n+1}\right)$ into (4.12) yields

$$
\begin{aligned}
& \alpha \operatorname{Re}\left\|\varphi_{\mathbf{u}}^{l+1}\right\|^{2}+\alpha \operatorname{Re}\left\|2 \varphi_{\mathbf{u}}^{l+1}-\varphi_{\mathbf{u}}^{l}\right\|^{2}+\frac{\lambda}{2}\left\|\varphi_{\sigma}^{l+1}\right\|^{2}+\frac{\lambda}{2}\left\|2 \varphi_{\sigma}^{l+1}-\varphi_{\sigma}^{l}\right\|^{2} \\
& +8 \alpha(1-\alpha) \Delta t \sum_{n=0}^{l}\left\|D\left(\varphi_{\mathbf{u}}^{n+1}\right)\right\|^{2}+2 \Delta t \sum_{n=0}^{l}\left\|\varphi_{\sigma}^{n+1}\right\|^{2} \\
& \leq 2 \alpha \operatorname{Re}\left\|\varphi_{\mathbf{u}}^{0}\right\|^{2}+\lambda\left\|\varphi_{\sigma}^{0}\right\|^{2}+2 \Delta t \sum_{n=0}^{l}\left[2 \alpha \operatorname{Re}\left(\epsilon_{5}+\epsilon_{6}+\cdots+\epsilon_{9}\right)\right]\left\|\varphi_{\mathbf{u}}^{n+1}\right\|^{2} \\
& +2 \triangle t \sum_{n=0}^{l}\left[\frac{\alpha d^{2} M^{2} R e}{2 \epsilon_{7}}+\frac{\lambda d^{3} M^{2}}{4 \beta_{4}}\right]\left\|2 \varphi_{\mathbf{u}}^{n}-\varphi_{\mathbf{u}}^{n-1}\right\|^{2}+2 \Delta t \sum_{n=0}^{l}\left[2 \alpha \left(\epsilon_{2}\right.\right. \\
& \left.\left.+\epsilon_{3}+\epsilon_{4}+\epsilon_{11}\right)\right]\left\|D\left(\varphi_{\mathbf{u}}^{n+1}\right)\right\|^{2}+2 \triangle t \sum_{n=0}^{l} \alpha \epsilon_{10}\left(\left\|D\left(\varphi_{\mathbf{u}}^{n}\right)\right\|^{2}+\left\|D\left(\varphi_{\mathbf{u}}^{n-1}\right)\right\|^{2}\right) \\
& +2 \Delta t \sum_{n=0}^{l}\left[\lambda\left(\beta_{1}+\beta_{4} \ldots+\beta_{13}+\frac{5 d^{3} C_{k}^{2} M^{2} \lambda}{16 \epsilon_{10} \alpha}+\frac{18 d^{2} \lambda K^{2} C_{k}^{2}}{\epsilon_{11} \alpha}\right)\right]\left\|\varphi_{\sigma}^{n+1}\right\|^{2} \\
& +2 \triangle t \sum_{n=0}^{l}\left(\beta_{2}+\beta_{3}\right)\left\|\varphi_{\sigma}^{n+1}\right\|^{2}+2 \triangle t \sum_{n=0}^{l} \frac{4 \lambda d^{2} M^{2}}{\beta_{10}}\left\|2 \varphi_{\sigma}^{n}-\varphi_{\sigma}^{n-1}\right\|^{2} \\
& +2 \triangle t \sum_{n=0}^{l} \frac{d C_{k}^{2} \alpha}{2 \epsilon_{2}}\left\|p^{n+1}-\Pi_{p}\left(p^{n+1}\right)\right\|^{2}+2 \Delta t \sum_{n=0}^{l} \frac{\operatorname{Re} \alpha}{2 \epsilon_{5}}\left\|\beth\left(\Pi_{\mathbf{u}}\left(\mathbf{u}^{n+1}\right)\right)-\partial_{t} \mathbf{u}^{n+1}\right\|^{2} \\
& +2 \Delta t \sum_{n=0}^{l} \frac{\lambda}{4 \beta_{1}}\left\|\beth\left(\Pi_{\sigma}\left(\sigma^{n+1}\right)\right)-\partial_{t} \sigma^{n+1}\right\|^{2}+2 \Delta t \sum_{n=0}^{l} \frac{4 d^{2} M^{2} \lambda}{\beta_{13}} \| \digamma\left(\sigma^{n+1}\right) \\
& -\sigma^{n+1}\left\|^{2}+2 \triangle t \sum_{n=0}^{l}\left[\frac{\alpha d^{2} M^{2} R e}{2 \epsilon_{6}}+\frac{\lambda d^{3} M^{2}}{4 \beta_{9}}\right]\right\| \digamma\left(\mathbf{u}^{n+1}\right)-\mathbf{u}^{n+1} \|^{2}
\end{aligned}
$$




$$
\begin{aligned}
& +2 \Delta t \sum_{n=0}^{l}\left(\frac{\alpha}{2 \epsilon_{3}}+\frac{1}{4 \beta_{2}}\right)\left\|\eta_{\sigma}^{n+1}\right\|^{2}+2 \Delta t \sum_{n=0}^{l}\left[\frac{\alpha \operatorname{Re} d^{2} M^{2}}{2 \epsilon_{8}}+\frac{\lambda d^{3} M^{2}}{4 \beta_{5}}\right]\left\|\digamma\left(\eta_{\mathbf{u}}^{n+1}\right)\right\|^{2} \\
& +2 \triangle t \sum_{n=0}^{l}\left[\frac{\alpha(1-\alpha)^{2}}{2 \epsilon_{4}}+\frac{\alpha^{2}}{\beta_{3}}+\frac{9 \alpha R e d^{2} K^{2} C_{k}^{2}}{2 \epsilon_{9}}+\frac{36 d^{2} \lambda K^{2} C_{k}^{2}}{\beta_{12}}\right]\left\|D\left(\eta_{\mathbf{u}}^{n+1}\right)\right\|^{2} \\
& +2 \Delta t \sum_{n=0}^{l} \frac{4 \lambda d^{2} M^{2}}{\beta_{11}}\left\|\digamma\left(\eta_{\sigma}^{n+1}\right)\right\|^{2} \\
& +2 \triangle t \sum_{n=0}^{l} \frac{9 \lambda d K^{2}}{4 \beta_{7}}\left\|\nabla \eta_{\sigma}^{n+1}\right\|^{2}+2 \Delta t \sum_{n=0}^{l} \frac{9 \lambda d^{2} K^{2} C_{i}^{2} h^{-2}}{16 \beta_{8}}\left\|\eta_{\sigma}^{n+1}\right\|^{2} .
\end{aligned}
$$

With the following choices: $\epsilon_{2}=\epsilon_{3}=\epsilon_{4}=\epsilon_{10}=\epsilon_{11}=\frac{1-\alpha}{6}, \epsilon_{5}=\epsilon_{6}=\epsilon_{7}=\epsilon_{8}=\epsilon_{9}=\frac{1}{20}$, $\beta_{1}=\beta_{4}=\cdots=\beta_{13}=\frac{1}{26 \lambda}, \beta_{2}=\beta_{3}=\frac{1}{26}, \mathbf{u}_{h}^{0}=\Pi_{\mathbf{u}}\left(\mathbf{u}_{0}\right)\left(\Rightarrow \varphi_{\mathbf{u}}^{0}=0\right), \sigma_{h}^{0}=\Pi_{\sigma}\left(\sigma_{0}\right)\left(\Rightarrow \varphi_{\sigma}^{0}=0\right)$, substituting these into (4.45) yields

$$
\begin{aligned}
& \alpha \operatorname{Re}\left\|\varphi_{\mathbf{u}}^{l+1}\right\|^{2}+\alpha \operatorname{Re}\left\|2 \varphi_{\mathbf{u}}^{l+1}-\varphi_{\mathbf{u}}^{l}\right\|^{2}+\frac{\lambda}{2}\left\|\varphi_{\sigma}^{l+1}\right\|^{2}+\frac{\lambda}{2}\left\|2 \varphi_{\sigma}^{l+1}-\varphi_{\sigma}^{l}\right\|^{2} \\
& +4 \alpha(1-\alpha) \Delta t \sum_{n=0}^{l}\left\|D\left(\varphi_{\mathbf{u}}^{n+1}\right)\right\|^{2}+\Delta t \sum_{n=0}^{l}\left\|\varphi_{\sigma}^{n+1}\right\|^{2} \\
& \leq \alpha \operatorname{Re} \triangle t \sum_{n=0}^{l}\left\|\varphi_{\mathbf{u}}^{n+1}\right\|^{2}+\triangle t \sum_{n=0}^{l}\left[20 d^{2} M^{2} R e+13 \lambda^{2} d^{3} M^{2}\right]\left\|2 \varphi_{\mathbf{u}}^{n}-\varphi_{\mathbf{u}}^{n-1}\right\|^{2} \\
& +\triangle t \sum_{n=0}^{l}\left[\frac{15 d^{3} C_{k}^{2} M^{2} \lambda^{2}}{4 \alpha(1-\alpha)}+\frac{216 d^{2} \lambda^{2} K^{2} C_{k}^{2}}{\alpha(1-\alpha)}\right]\left\|\varphi_{\sigma}^{n+1}\right\|^{2}+208 \lambda^{2} d^{2} M^{2} \Delta t \sum_{n=0}^{l} \| 2 \varphi_{\sigma}^{n} \\
& -\varphi_{\sigma}^{n-1}\left\|^{2}+\Delta t \sum_{n=0}^{l} \frac{6 \alpha d C_{k}^{2}}{1-\alpha}\right\| p^{n+1}-\Pi_{p}\left(p^{n+1}\right)\left\|^{2}+20 \operatorname{Re} \alpha \Delta t \sum_{n=0}^{l}\right\| \beth\left(\Pi_{\mathbf{u}}\left(\mathbf{u}^{n+1}\right)\right) \\
& -\partial_{t} \mathbf{u}^{n+1}\left\|^{2}+13 \lambda^{2} \Delta t \sum_{n=0}^{l}\right\| \beth\left(\Pi_{\sigma}\left(\sigma^{n+1}\right)\right)-\partial_{t} \sigma^{n+1} \|^{2} \\
& +208 d^{2} M^{2} \lambda^{2} \Delta t \sum_{n=0}^{l}\left\|\digamma\left(\sigma^{n+1}\right)-\sigma^{n+1}\right\|^{2}+\left[\frac{6 \alpha}{1-\alpha}+13\right] \Delta t \sum_{n=0}^{l}\left\|\eta_{\sigma}^{n+1}\right\|^{2} \\
& +\left[20 \alpha d^{2} M^{2} R e+13 \lambda^{2} d^{3} M^{2}\right] \Delta t \sum_{n=0}^{l}\left\|\digamma\left(\mathbf{u}^{n+1}\right)-\mathbf{u}^{n+1}\right\|^{2}+[6 \alpha(1-\alpha) \\
& \left.+52 \lambda \alpha^{2}+180 \alpha \operatorname{Red}^{2} K^{2} C_{k}^{2}+1872 d^{2} \lambda^{2} K^{2} C_{k}^{2}\right] \triangle t \sum_{n=0}^{l}\left\|D\left(\eta_{\mathbf{u}}^{n+1}\right)\right\|^{2} \\
& +\left[20 \alpha \operatorname{Red}^{2} M^{2}+13 \lambda^{2} d^{3} M^{2}\right] \Delta t \sum_{n=0}^{l}\left\|\digamma\left(\eta_{\mathbf{u}}^{n+1}\right)\right\|^{2} \\
& +208 \lambda^{2} d^{2} M^{2} \Delta t \sum_{n=0}^{l}\left\|\digamma\left(\eta_{\sigma}^{n+1}\right)\right\|^{2} \\
& +117 \lambda^{2} d K^{2} \triangle t \sum_{n=0}^{l}\left\|\nabla \eta_{\sigma}^{n+1}\right\|^{2}+\frac{117 \lambda^{2} d^{2} K^{2} C_{i}^{2} h^{-2}}{4} \Delta t \sum_{n=0}^{l}\left\|\eta_{\sigma}^{n+1}\right\|^{2} .
\end{aligned}
$$


We now apply the approximation properties (2.4)-(2.6) to the terms on the RHS of (4.46). Using elements of order $k$ for velocity, elements of order $m$ for stress, and elements of order $q$ for pressure, we have

$$
\begin{aligned}
& \Delta t \sum_{n=0}^{l} \frac{6 \alpha d C_{k}^{2}}{1-\alpha}\left\|p^{n+1}-\Pi_{p}\left(p^{n+1}\right)\right\|^{2} \leq \frac{6 \alpha d C_{k}^{2} C_{i p}^{2}}{1-\alpha} h^{2 q+2}\|p\|_{0, q+1}^{2}, \\
& {\left[\frac{6 \alpha}{1-\alpha}+13\right] \Delta t \sum_{n=0}^{l}\left\|\eta_{\sigma}^{n+1}\right\|^{2} \leq\left[\frac{6 \alpha}{1-\alpha}+13\right] C_{i p}^{2} h^{2 m+2}\|\sigma\|_{0, m+1}^{2},} \\
& {\left[6 \alpha(1-\alpha)+52 \lambda \alpha^{2}+d^{2} K^{2} C_{k}^{2}\left(180 \alpha R e+1872 \lambda^{2}\right)\right] \Delta t \sum_{n=0}^{l}\left\|D\left(\eta_{\mathbf{u}}^{n+1}\right)\right\|^{2}} \\
& \quad \leq\left[6 \alpha(1-\alpha)+52 \lambda \alpha^{2}+d^{2} K^{2} C_{k}^{2}\left(180 \alpha R e+1872 \lambda^{2}\right)\right] C_{i p}^{2} h^{2 k}\|\mathbf{u}\|_{0, k+1}^{2}, \\
& {\left[20 \alpha \operatorname{Re} d^{2} M^{2}+13 \lambda^{2} d^{3} M^{2}\right] \triangle t \sum_{n=0}^{l}\left\|\digamma\left(\eta_{\mathbf{u}}^{n+1}\right)\right\|^{2}} \\
& \quad \leq\left[20 \alpha \operatorname{Red}{ }^{2} M^{2}+13 \lambda^{2} d^{3} M^{2}\right] C_{i p}^{2} h^{2 k+2}\|\mathbf{u}\|_{0, k+1}^{2}, \\
& \frac{13 \lambda^{2} d^{3} M^{2}}{4} \Delta t \sum_{n=0}^{l}\left\|\nabla \digamma\left(\eta_{\mathbf{u}}^{n+1}\right)\right\|^{2} \leq \frac{13 \lambda^{2} d^{3} M^{2}}{4} C_{i p}^{2} h^{2 k}\|\mathbf{u}\|_{0, k+1}^{2}, \\
& 208 \lambda^{2} d^{2} M^{2} \Delta t \sum_{n=0}^{l}\left\|\digamma\left(\eta_{\sigma}^{n+1}\right)\right\|^{2} \leq 208 \lambda^{2} d^{2} M^{2} C_{i p}^{2} h^{2 m+2}\|\sigma\|_{0, m+1}^{2}, \\
& \leq 117 \lambda^{2} d K^{2}\left[1+\frac{d C_{i}^{2}}{4}\right] C_{i p}^{2} h^{2 m}\|\sigma\|_{0, m+1}^{2} . \\
& 117 \lambda^{2} d K^{2} \Delta t \sum_{n=0}^{l}\left\|\nabla \eta_{\sigma}^{n+1}\right\|^{2}+\frac{117 \lambda^{2} d^{2} K^{2} C_{i}^{2} h^{-2}}{4} \Delta t \sum_{n=0}^{l}\left\|\eta_{\sigma}^{n+1}\right\|^{2}
\end{aligned}
$$

In view of the truncation error (2.11) and the interpolation properties (2.4), we can obtain

$$
\begin{aligned}
\| & \beth\left(\Pi_{\mathbf{u}}\left(\mathbf{u}^{n+1}\right)\right)-\partial_{t} \mathbf{u}^{n+1} \| \\
\quad & =\left\|\beth\left(\Pi_{\mathbf{u}}\left(\mathbf{u}\left(t_{n+1}\right)\right)\right)-\partial_{t} \mathbf{u}\left(t_{n+1}\right)\right\| \\
& \leq\left\|\Pi_{\mathbf{u}}\left(\beth\left(\mathbf{u}\left(t_{n+1}\right)\right)-\partial_{t} \mathbf{u}\left(t_{n+1}\right)\right)\right\|+\left\|\partial_{t}\left(\Pi_{\mathbf{u}}\left(\mathbf{u}\left(t_{n+1}\right)\right)-\mathbf{u}\left(t_{n+1}\right)\right)\right\| \\
& \leq C_{T}(\Delta t)^{3 / 2}\left\|\partial_{t}^{3} \mathbf{u}\right\|_{L^{2}\left(t_{n-2}, t_{n} ; L^{2}(\Omega)^{d}\right)}+C_{i p} \frac{h^{k}}{\sqrt{\Delta t}}\left\|\partial_{t} \mathbf{u}\right\|_{L^{2}\left(0, T ; H^{k+1}(\Omega)^{d}\right)} .
\end{aligned}
$$

Then we have

$$
\begin{gathered}
20 \operatorname{Re} \alpha \Delta t \sum_{n=0}^{l}\left\|\beth\left(\Pi_{\mathbf{u}}\left(\mathbf{u}^{n+1}\right)\right)-\partial_{t} \mathbf{u}^{n+1}\right\|^{2}+13 \lambda^{2} \Delta t \sum_{n=0}^{l}\left\|\beth\left(\Pi_{\sigma}\left(\sigma^{n+1}\right)\right)-\partial_{t} \sigma^{n+1}\right\|^{2} \\
\leq 40 \operatorname{Re} \alpha C_{T}^{2} \Delta t^{4}\left\|\partial_{t}^{3} \mathbf{u}\right\|_{L^{2}\left(0, T ; L^{2}(\Omega)^{d}\right)}^{2}+26 \lambda^{2} C_{T}^{2} \Delta t^{4}\left\|\partial_{t}^{3} \sigma\right\|_{\left.L^{2}\left(0, T ; L^{2}(\Omega)\right)^{d \times d}\right)}^{2} \\
+40 \operatorname{Re} \alpha C_{i p}^{2} h^{2 k}\left\|\partial_{t} \mathbf{u}\right\|_{L^{2}\left(0, T ; H^{k+1}(\Omega)^{d}\right)}^{2}+26 \lambda^{2} C_{i p}^{2} h^{2 m}\left\|\partial_{t} \sigma\right\|_{L^{2}\left(0, T ; H^{m+1}(\Omega)^{d \times d}\right)}^{2} .
\end{gathered}
$$


Similarly, using the truncation error (2.12), we get

$$
\begin{aligned}
{[20 \alpha} & \left.d^{2} M^{2} \operatorname{Re}+13 \lambda^{2} d^{3} M^{2}\right] \triangle t \sum_{n=0}^{l}\left\|\digamma\left(\mathbf{u}^{n+1}\right)-\mathbf{u}^{n+1}\right\|^{2} \\
& +208 d^{2} M^{2} \lambda^{2} \Delta t \sum_{n=0}^{l}\left\|\digamma\left(\sigma^{n+1}\right)-\sigma^{n+1}\right\|^{2} \\
\leq & {\left.\left[20 \alpha d^{2} M^{2} R e+13 \lambda^{2} d^{3} M^{2}\right] C_{T}^{2} \Delta t^{4}\left\|\partial_{t}^{2} \mathbf{u}\right\|_{L^{2}\left(0, T ; L^{2}(\Omega)\right.}^{2}\right) } \\
& \left.+208 d^{2} M^{2} \lambda^{2} C_{T}^{2} \triangle t^{4}\left\|\partial_{t}^{2} \sigma\right\|_{L^{2}\left(0, T ; L^{2}(\Omega)\right.}^{2} d \times d\right)^{\cdot}
\end{aligned}
$$

Combining inequalities (4.47)-(4.56) with (4.46) yields

$$
\begin{aligned}
& \alpha \operatorname{Re}\left\|\varphi_{\mathbf{u}}^{l+1}\right\|^{2}+\alpha \operatorname{Re}\left\|2 \varphi_{\mathbf{u}}^{l+1}-\varphi_{\mathbf{u}}^{l}\right\|^{2}+\frac{\lambda}{2}\left\|\varphi_{\sigma}^{l+1}\right\|^{2}+\frac{\lambda}{2}\left\|2 \varphi_{\sigma}^{l+1}-\varphi_{\sigma}^{l}\right\|^{2} \\
& +4 \alpha(1-\alpha) \Delta t \sum_{n=0}^{l}\left\|D\left(\varphi_{\mathbf{u}}^{n+1}\right)\right\|^{2}+\Delta t \sum_{n=0}^{l}\left\|\varphi_{\sigma}^{n+1}\right\|^{2} \\
& \leq \alpha \operatorname{Re} \Delta t \sum_{n=0}^{l}\left\|\varphi_{\mathbf{u}}^{n+1}\right\|^{2}+\left[20 d^{2} M^{2} \operatorname{Re}+13 \lambda^{2} d^{3} M^{2}\right] \Delta t \sum_{n=0}^{l} \| 2 \varphi_{\mathbf{u}}^{n} \\
& -\varphi_{\mathbf{u}}^{n-1}\left\|^{2}+\Delta t \sum_{n=0}^{l}\left[\frac{15 d^{3} C_{k}^{2} M^{2} \lambda^{2}}{4 \alpha(1-\alpha)}+\frac{216 d^{2} \lambda^{2} K^{2} C_{k}^{2}}{\alpha(1-\alpha)}\right]\right\| \varphi_{\sigma}^{n+1} \|^{2} \\
& +208 \lambda^{2} d^{2} M^{2} \triangle t \sum_{n=0}^{l}\left\|2 \varphi_{\sigma}^{n}-\varphi_{\sigma}^{n-1}\right\|^{2}+\frac{6 \alpha d C_{k}^{2} C_{i p}^{2}}{1-\alpha} h^{2 q+2}\|p\|_{0, q+1}^{2} \\
& +\left[\frac{6 \alpha}{1-\alpha}+13+208 \lambda^{2} d^{2} M^{2}\right] C_{i p}^{2} h^{2 m+2}\|\sigma\|_{0, m+1}^{2}+\left[6 \alpha(1-\alpha)+52 \lambda \alpha^{2}\right. \\
& \left.+180 \alpha \operatorname{Red}^{2} K^{2} C_{k}^{2}+1872 d^{2} \lambda^{2} K^{2} C_{k}^{2}+\frac{13 \lambda^{2} d^{3} M^{2}}{4}\right] C_{i p}^{2} h^{2 k}\|\mathbf{u}\|_{0, k+1}^{2} \\
& +\left[20 \alpha \operatorname{Red}^{2} M^{2}+13 \lambda^{2} d^{3} M^{2}\right] C_{i p}^{2} h^{2 k+2}\|\mathbf{u}\|_{0, k+1}^{2}+117 \lambda^{2} K^{2} d[1 \\
& \left.\left.+\frac{d C_{i}^{2}}{4}\right] C_{i p}^{2} h^{2 m}\|\sigma\|_{0, m+1}^{2}+40 \operatorname{Re} \alpha C_{i p}^{2} h^{2 k}\left\|\partial_{t} \mathbf{u}\right\|_{L^{2}\left(0, T ; H^{k+1}(\Omega) d\right.}^{2}\right) \\
& +\left[20 \alpha d^{2} M^{2} R e+13 \lambda^{2} d^{3} M^{2}\right] C_{T}^{2} \triangle t^{4}\left\|\partial_{t}^{2} u\right\|_{L^{2}\left(0, T ; L^{2}(\Omega) d\right.}^{2} \\
& \left.+40 \operatorname{Re} \alpha C_{T}^{2} \Delta t^{4}\left\|\partial_{t}^{3} \mathbf{u}\right\|_{L^{2}\left(0, T ; L^{2}(\Omega)^{d}\right)}^{2}+26 \lambda^{2} C_{i p}^{2} h^{2 m}\left\|\partial_{t} \sigma\right\|_{L^{2}\left(0, T ; H^{m+1}(\Omega) d \times d\right.}^{2}\right) \\
& +26 \lambda^{2} C_{T}^{2} \triangle t^{4}\left\|\partial_{t}^{3} \sigma\right\|_{L^{2}\left(0, T ; L^{2}(\Omega) d \times d\right)}^{2} \\
& \left.+208 d^{2} M^{2} \lambda^{2} C_{T}^{2} \triangle t^{4}\left\|\partial_{t}^{2} \sigma\right\|_{L^{2}\left(0, T ; L^{2}(\Omega)\right.}^{2} d \times d\right) .
\end{aligned}
$$

In order to use the discrete Gronwall Lemma 2.3, here we set

$$
\begin{aligned}
& a_{l}=\alpha \operatorname{Re}\left\|\varphi_{\mathbf{u}}^{l+1}\right\|^{2}+\alpha \operatorname{Re}\left\|2 \varphi_{\mathbf{u}}^{l+1}-\varphi_{\mathbf{u}}^{l}\right\|^{2}+\frac{\lambda}{2}\left\|\varphi_{\sigma}^{l+1}\right\|^{2}+\frac{\lambda}{2}\left\|2 \varphi_{\sigma}^{l+1}-\varphi_{\sigma}^{l}\right\|^{2}, \\
& b_{n}=4 \alpha(1-\alpha)\left\|D\left(\varphi_{\mathbf{u}}^{n+1}\right)\right\|^{2}+\left\|\varphi_{\sigma}^{n+1}\right\|^{2},
\end{aligned}
$$




$$
\begin{aligned}
& a_{n}=\alpha \operatorname{Re}\left\|\varphi_{\mathbf{u}}^{n+1}\right\|^{2}+\alpha \operatorname{Re}\left\|2 \varphi_{\mathbf{u}}^{n+1}-\varphi_{\mathbf{u}}^{n}\right\|^{2}+\frac{\lambda}{2}\left\|\varphi_{\sigma}^{n+1}\right\|^{2}+\frac{\lambda}{2}\left\|2 \varphi_{\sigma}^{n+1}-\varphi_{\sigma}^{n}\right\|^{2}, \\
& \gamma_{n}=\max \left\{1, \frac{d^{2} M^{2}}{\alpha}\left(20+\frac{13 \lambda^{2} d}{\operatorname{Re}}\right), \frac{d^{2} C_{k}^{2} \lambda}{\alpha(1-\alpha)}\left(\frac{15 d M^{2}}{2}+432 K^{2}\right), 416 \lambda d^{2} M^{2}\right\}, \\
& c_{n}=0, \quad \zeta_{n}=\frac{1}{1-\Delta t \gamma_{n}}, \\
& H=\text { other (non-summing) terms on the RHS of (4.57). }
\end{aligned}
$$

For $\gamma_{n} \Delta t \leq \frac{1}{2}$, using the discrete Gronwall lemma to (4.57) yields Theorem 4.1.

We will deduce that the induction assumption IH1 (3.3) is right for any $n=0,1,2, \ldots, N$, by mathematical induction.

Lemma 4.2 Let $\left(\mathbf{u}_{h}^{l}, p_{h}^{l}, \sigma_{h}^{l}\right) \in X_{h} \times Q_{h} \times S_{h}$ satisfy (3.1a)-(3.1c) for each $l \in\{0,1,2, \ldots, N\}$. There is a bounded constant $K$ such that

$$
\left\|\sigma_{h}^{l}\right\|_{\infty} \leq K, \quad\left\|\mathbf{u}_{h}^{l}\right\|_{\infty} \leq K .
$$

Proof Since $\left\|\sigma_{h}^{0}\right\|_{\infty}=\left\|\Pi_{\sigma}\left(\sigma_{0}\right)\right\|_{\infty} \leq\left\|\sigma_{0}\right\|_{\infty} \leq M \leq K$. Now we assume that (4.58) holds true for $n=0,1,2, \ldots, l$. By interpolation properties, inverse estimates, the regularity assumption (2.3) of $\sigma$, and result (4.3), we have that

$$
\begin{aligned}
\left\|\sigma_{h}^{l+1}\right\|_{\infty} & \leq\left\|\left(\sigma_{h}^{l+1}-\Pi_{\sigma}\left(\sigma^{l+1}\right)\right)+\left(\Pi_{\sigma}\left(\sigma^{l+1}\right)-\sigma^{l+1}\right)+\sigma^{l+1}\right\|_{\infty} \\
& \leq\left\|\varphi_{\sigma}^{l+1}\right\|_{\infty}+\left\|\eta_{\sigma}^{l+1}\right\|_{\infty}+\left\|\sigma^{l+1}\right\|_{\infty} \\
& \leq C h^{-\frac{d}{2}}\left\|\varphi_{\sigma}^{l+1}\right\|+C h^{-\frac{d}{2}}\left\|\eta_{\sigma}^{l+1}\right\|+M \\
& \leq C\left(\Delta t^{2} h^{-\frac{d}{2}}+h^{k-\frac{d}{2}}+h^{m-\frac{d}{2}}+h^{q+1-\frac{d}{2}}\right)+M .
\end{aligned}
$$

We can see that the expression $C\left(\Delta t^{2} h^{-\frac{d}{2}}+h^{k-\frac{d}{2}}+h^{m-\frac{d}{2}}+h^{q+1-\frac{d}{2}}\right)$ is independent of $l$. Hence, if we set $k, m \geq \frac{d}{2}, q+1 \geq \frac{d}{2}$, and choose $h, \Delta t$ such that

$$
h^{k-\frac{d}{2}}, h^{m-\frac{d}{2}}, h^{q+1-\frac{d}{2}} \leq \frac{1}{C}, \quad \triangle t \leq \frac{1}{C} h^{\frac{d}{4}},
$$

then from (4.59)

$$
\left\|\sigma_{h}^{l+1}\right\|_{\infty} \leq 4+M=K
$$

Similarly, we get $\left\|\mathbf{u}_{h}^{l+1}\right\|_{\infty} \leq 4+M=K$.

Theorem 4.3 Under the conditions of Theorem 4.1 and $\Delta t \leq C_{0} h^{\frac{d}{4}}$, we have

$$
\begin{gathered}
\alpha \operatorname{Re}\left\|\mathbf{u}^{l}-\mathbf{u}_{h}^{l}\right\|^{2}+\frac{\lambda}{2}\left\|\sigma^{l}-\sigma_{h}^{l}\right\|^{2}+\Delta t \sum_{n=0}^{l}\left\|\sigma^{n+1}-\sigma_{h}^{n+1}\right\|^{2} \\
+4 \alpha(1-\alpha) \Delta t \sum_{n=0}^{l}\left\|D\left(\mathbf{u}^{n+1}-\mathbf{u}_{h}^{n+1}\right)\right\|^{2} \leq W(\Delta t, h) .
\end{gathered}
$$


Proof We add both sides of (4.3) with

$$
\begin{aligned}
\text { Extraterms }= & \alpha \operatorname{Re}\left\|\eta_{\mathbf{u}}^{l+1}\right\|^{2}+\alpha \operatorname{Re}\left\|2 \eta_{\mathbf{u}}^{l+1}-\eta_{\mathbf{u}}^{l}\right\|^{2}+\frac{\lambda}{2}\left\|2 \eta_{\sigma}^{l+1}-\eta_{\sigma}^{l}\right\|^{2} \\
& +\frac{\lambda}{2}\left\|\eta_{\sigma}^{l+1}\right\|^{2}+\Delta t \sum_{n=0}^{l}\left[4 \alpha(1-\alpha)\left\|D\left(\eta_{\mathbf{u}}^{n+1}\right)\right\|^{2}+\left\|\eta_{\sigma}^{n+1}\right\|^{2}\right],
\end{aligned}
$$

and apply the triangle inequality for the left-hand side. Noticing that the upcoming terms are already contained in the RHS of the model, we obtain the a priori error estimate (4.60).

Theorem 4.4 Under the conditions of Theorem 4.3, for any $0 \leq l \leq N-1$, there is a positive constant $C_{1}$ independent of $\triangle t$ and $h$ such that

$$
\beta^{2} \triangle t \sum_{n=0}^{l}\left\|p^{n+1}-p_{h}^{n+1}\right\|^{2} \leq C_{1} W(\triangle t, h) .
$$

Proof As $V_{h} \subset X_{h}$, for all $\mathbf{v}_{h} \in V_{h}$, we have from (4.6a)

$$
\begin{aligned}
\operatorname{Re}(\beth & \left.\left(e_{\mathbf{u}}^{n+1}\right), \mathbf{v}_{h}\right)+\left(e_{\sigma}^{n+1}, D\left(\mathbf{v}_{h}\right)\right)+(1-\alpha)\left(\nabla\left(e_{\mathbf{u}}^{n+1}\right), \nabla\left(\mathbf{v}_{h}\right)\right) \\
& +\operatorname{Rec}\left(\digamma\left(e_{\mathbf{u}}^{n+1}\right), \mathbf{u}^{n+1}, \mathbf{v}_{h}\right)+\operatorname{Rec}\left(\digamma\left(\mathbf{u}_{h}^{n+1}\right), e_{\mathbf{u}}^{n+1}, \mathbf{v}_{h}\right) \\
= & \left(p^{n+1}-\lambda_{h}^{n+1}, \nabla \cdot \mathbf{v}_{h}\right)+\operatorname{Re}\left(\beth\left(\mathbf{u}^{n+1}\right)-\partial_{t} \mathbf{u}^{n+1}, \mathbf{v}_{h}\right) \\
& +\operatorname{Rec}\left(\digamma\left(\mathbf{u}^{n+1}\right)-\mathbf{u}^{n+1}, \mathbf{u}^{n+1}, \mathbf{v}_{h}\right),
\end{aligned}
$$

where $\lambda_{h}^{n+1}$ is an approximation to $p^{n+1}$. Dividing by $\left\|\nabla \mathbf{v}_{h}\right\|$, using the Cauchy-Schwarz inequality and the Poincaré inequality lead to

$$
\begin{aligned}
\operatorname{Re} \frac{\left|\left(\beth\left(e_{\mathbf{u}}^{n+1}\right), \mathbf{v}_{h}\right)\right|}{\left\|\nabla\left(\mathbf{v}_{h}\right)\right\|} \leq & \left\|e_{\sigma}^{n+1}\right\|+(1-\alpha)\left\|\nabla\left(e_{\mathbf{u}}^{n+1}\right)\right\| \\
& +\operatorname{Re} C_{p} \sqrt{d}\left\|\digamma\left(e_{\mathbf{u}}^{n+1}\right)\right\|\left\|\nabla \mathbf{u}^{n+1}\right\|_{\infty}+\operatorname{Re} \sqrt{d}\left\|\digamma\left(\mathbf{u}_{h}^{n+1}\right)\right\|_{\infty}\left\|e_{\mathbf{u}}^{n+1}\right\| \\
& +\sqrt{d}\left\|p^{n+1}-\lambda_{h}^{n+1}\right\|+\operatorname{Re} C_{p}\left\|\beth\left(\mathbf{u}^{n+1}\right)-\partial_{t} \mathbf{u}^{n+1}\right\| \\
& +\operatorname{Re}_{p}\left\|\digamma\left(\mathbf{u}^{n+1}\right)-\mathbf{u}^{n+1}\right\|\left\|\nabla \mathbf{u}^{n+1}\right\|_{\infty} .
\end{aligned}
$$

Applying Lemma 2.2 and the regularity assumption (2.3) and taking the supremum over $\mathbf{v}_{h} \in V_{h}$ yield

$$
\begin{aligned}
\operatorname{Re}\left\|\beth\left(e_{\mathbf{u}}^{n+1}\right)\right\|_{X_{h}^{\prime}} \leq & \left\|e_{\sigma}^{n+1}\right\|+(1-\alpha)\left\|\nabla\left(e_{\mathbf{u}}^{n+1}\right)\right\|+\operatorname{Re} C_{p} M \sqrt{d}\left\|\digamma\left(e_{\mathbf{u}}^{n+1}\right)\right\| \\
& +3 \operatorname{Re} K \sqrt{d}\left\|e_{\mathbf{u}}^{n+1}\right\|+\sqrt{d}\left\|p^{n+1}-\lambda_{h}^{n+1}\right\| \\
& +\operatorname{ReC}_{p}\left\|\beth\left(\mathbf{u}^{n+1}\right)-\partial_{t} \mathbf{u}^{n+1}\right\|+\operatorname{Re} C_{p} M\left\|\digamma\left(\mathbf{u}^{n+1}\right)-\mathbf{u}^{n+1}\right\| .
\end{aligned}
$$


Splitting $p^{n+1}-p_{h}^{n+1}=\left(p^{n+1}-\lambda_{h}^{n+1}\right)+\left(\lambda_{h}^{n+1}-p_{h}^{n+1}\right)$, we get from (4.63) that

$$
\begin{aligned}
\left(\lambda_{h}^{n+1}\right. & \left.-p_{h}^{n+1}, \nabla \cdot \mathbf{v}_{h}\right) \\
= & -\left(p^{n+1}-\lambda_{h}^{n+1}, \nabla \cdot \mathbf{v}_{h}\right)+\operatorname{Re}\left(\beth\left(e_{\mathbf{u}}^{n+1}\right), \mathbf{v}_{h}\right) \\
& +\left(e_{\sigma}^{n+1}, D\left(\mathbf{v}_{h}\right)\right)+(1-\alpha)\left(\nabla\left(e_{\mathbf{u}}^{n+1}\right), \nabla\left(\mathbf{v}_{h}\right)\right)+\operatorname{Rec}\left(\digamma\left(e_{\mathbf{u}}^{n+1}\right), \mathbf{u}^{n+1}, \mathbf{v}_{h}\right) \\
& +\operatorname{Rec}\left(\digamma\left(\mathbf{u}_{h}^{n+1}\right), e_{\mathbf{u}}^{n+1}, \mathbf{v}_{h}\right)-\operatorname{Re}\left(\beth\left(\mathbf{u}^{n+1}\right)-\partial_{t} \mathbf{u}^{n+1}, \mathbf{v}_{h}\right) \\
& +\operatorname{Rec}\left(\digamma\left(\mathbf{u}^{n+1}\right)-\mathbf{u}^{n+1}, \mathbf{u}^{n+1}, \mathbf{v}_{h}\right) .
\end{aligned}
$$

Combining the inf-sup condition (2.7) with (4.64)-(4.65), we have

$$
\begin{aligned}
\beta\left\|\lambda_{h}^{n+1}-p_{h}^{n+1}\right\| \leq & 2 \sqrt{d}\left\|\lambda_{h}^{n+1}-p^{n+1}\right\|+2\left\|e_{\sigma}^{n+1}\right\|+2(1-\alpha)\left\|\nabla\left(e_{\mathbf{u}}^{n+1}\right)\right\| \\
& +2 \sqrt{d} \operatorname{Re} C_{p}^{2} M\left\|\nabla\left(\digamma\left(e_{\mathbf{u}}^{n+1}\right)\right)\right\|+2 \operatorname{Re} C_{p}\left\|\beth\left(\mathbf{u}^{n+1}\right)-\partial_{t} \mathbf{u}^{n+1}\right\| \\
& +6 \sqrt{d} \operatorname{ReKC} C_{p}\left\|\nabla e_{\mathbf{u}}^{n+1}\right\|+2 \operatorname{Re} C_{p} M\left\|\digamma\left(\mathbf{u}^{n+1}\right)-\mathbf{u}^{n+1}\right\| .
\end{aligned}
$$

Applying the triangle inequality to (4.67) yields

$$
\begin{aligned}
\beta\left\|p^{n+1}-p_{h}^{n+1}\right\| \leq & (1+2 \sqrt{d})\left\|\lambda_{h}^{n+1}-p^{n+1}\right\|+2 \sqrt{d} \operatorname{Re} C_{p}^{2} M\left\|\nabla\left(\digamma\left(e_{\mathbf{u}}^{n+1}\right)\right)\right\| \\
& +2 \operatorname{Re} C_{p}\left\|\beth\left(\mathbf{u}^{n+1}\right)-\partial_{t} \mathbf{u}^{n+1}\right\|+\left[2-2 \alpha+6 \sqrt{d} \operatorname{ReKC} C_{p}\right]\left\|\nabla\left(e_{\mathbf{u}}^{n+1}\right)\right\| \\
& +2\left\|e_{\sigma}^{n+1}\right\|+2 \operatorname{Re} C_{p} M\left\|\digamma\left(\mathbf{u}^{n+1}\right)-\mathbf{u}^{n+1}\right\| .
\end{aligned}
$$

Applying $\left(a_{1}+\cdots+a_{6}\right)^{2} \leq 6\left(a_{1}^{2}+\cdots+a_{6}^{2}\right)$ to above equation, summing (4.68) with respect to $n$ from 0 to $l$, and multiplying both sides of the equation by $\Delta t$ yield

$$
\begin{aligned}
& \beta^{2} \triangle t \sum_{n=0}^{l}\left\|p^{n+1}-p_{h}^{n+1}\right\|^{2} \\
& \leq 6(1+2 \sqrt{d})^{2} \Delta t \sum_{n=0}^{l}\left\|\lambda_{h}^{n+1}-p^{n+1}\right\|^{2} \\
& +6\left(2-2 \alpha+6 \sqrt{d} \operatorname{ReK} C_{p}\right)^{2} C_{k}^{2} \Delta t \sum_{n=0}^{l}\left\|D\left(e_{\mathbf{u}}^{n+1}\right)\right\|^{2} \\
& \quad+24 d R e^{2} C_{p}^{4} M^{2} C_{k}^{2} \triangle t \sum_{n=0}^{l}\left\|D\left(\digamma\left(e_{\mathbf{u}}^{n+1}\right)\right)\right\|^{2} \\
& +24 \operatorname{Re}^{2} C_{p}^{2} \triangle t \sum_{n=0}^{l}\left\|\beth\left(\mathbf{u}^{n+1}\right)-\partial_{t} \mathbf{u}^{n+1}\right\|^{2} \\
& +24 \Delta t \sum_{n=0}^{l}\left\|e_{\sigma}^{n+1}\right\|^{2}+24 R e^{2} C_{p}^{2} M^{2} \triangle t \sum_{n=0}^{l}\left\|\digamma\left(\mathbf{u}^{n+1}\right)-\mathbf{u}^{n+1}\right\|^{2}
\end{aligned}
$$

Making use of the approximation property (2.5) of pressure, the error estimate $\left\|\sigma-\sigma_{h}\right\|_{0,0}^{2}$ and $\left\|D\left(\mathbf{u}-\mathbf{u}_{h}\right)\right\|_{0,0}^{2}$ in Theorem 4.3, the truncation errors (2.11) and (2.12) of the temporal discretion, we can derive the required result (4.62). 
If the domain $\Omega \subset \mathbb{R}^{2}$, then we can use the MINI elements $\left(P_{1} b, P_{1}\right)$ pair, which satisfies the discrete inf-sup condition (2.7), to approximate the velocity $\mathbf{u}$ and pressure $p$ and the $P_{1}$ discontinuous element to approximate the stress $\sigma$, that is, $k=1, q=1, m=1$, we have the following convergence result.

Corollary 4.5 Under the conditions of Theorem 4.3 and using the pair $\left(P_{1} b, P_{1}, P_{1} d c\right)$ elements to approximate $(\mathbf{u}, p, \sigma)$, there is a positive constant $C_{2}$ independent of $\Delta t$ and $h$ such that

$$
\begin{aligned}
& \alpha R e\left\|\mathbf{u}^{l}-\mathbf{u}_{h}^{l}\right\|^{2}+\frac{\lambda}{2}\left\|\sigma^{l}-\sigma_{h}^{l}\right\|^{2}+\Delta t \sum_{n=0}^{l}\left\|\sigma^{n+1}-\sigma_{h}^{n+1}\right\|^{2} \\
& \quad+4 \alpha(1-\alpha) \Delta t \sum_{n=0}^{l}\left\|D\left(\mathbf{u}^{n+1}-\mathbf{u}_{h}^{n+1}\right)\right\|^{2}+\Delta t \sum_{n=0}^{l}\left\|p^{n+1}-p_{h}^{n+1}\right\|^{2} \\
& \leq C_{2}\left(\Delta t^{4}+h^{2}\right) .
\end{aligned}
$$

Corollary 4.6 If the domain $\Omega \subset \mathbb{R}^{d}, d=2,3$, and making use of Taylor-Hood $\left(P_{2}, P_{1}\right)$ elements to approximate velocity $\mathbf{u}$ and pressure $p$, and $P_{2}$ discontinuous element for $\sigma$, that is, $k=2, q=1, m=2$, we have

$$
\begin{aligned}
& \alpha \operatorname{Re}\left\|\mathbf{u}^{l}-\mathbf{u}_{h}^{l}\right\|^{2}+\frac{\lambda}{2}\left\|\sigma^{l}-\sigma_{h}^{l}\right\|^{2}+\Delta t \sum_{n=0}^{l}\left\|\sigma^{n+1}-\sigma_{h}^{n+1}\right\|^{2} \\
& \quad+4 \alpha(1-\alpha) \Delta t \sum_{n=0}^{l}\left\|D\left(\mathbf{u}^{n+1}-\mathbf{u}_{h}^{n+1}\right)\right\|^{2}+\Delta t \sum_{n=0}^{l}\left\|p^{n+1}-p_{h}^{n+1}\right\|^{2} \\
& \leq C_{3}\left(\Delta t^{4}+h^{4}\right) .
\end{aligned}
$$

\section{Numerical experiments}

In this section, some numerical tests are performed by using FreeFem ++ [41] to confirm our theoretical analysis.

\subsection{Analytical solution}

A known analytical solution example is used to verify theoretical convergence rates of the linearized scheme. We choose the final time $T=0.1$ and computer domain $\Omega=[0,1]^{2}$. Same as $[8,39]$, the right-hand side function is added to the constitutive equation (1.1b) such that the analytical solutions $(\mathbf{u}, p, \sigma)$ are taken as follows:

$$
\begin{aligned}
& u_{1}(x, y)=10 x^{2}(x-1)^{2} y(y-1)(2 y-1) \cos (t), \\
& u_{2}(x, y)=-10 x(x-1)(2 x-1) y^{2}(y-1)^{2} \cos (t), \\
& p(x, y)=10(2 x-1)(2 y-1) \cos (t), \\
& \sigma=2 \alpha D(\mathbf{u}), \quad \mathbf{u}=\left(u_{1}, u_{2}\right),
\end{aligned}
$$

with the parameter $\lambda=1.0, \alpha=0.5, a=0, \operatorname{Re}=1.0$. It is easy to see that the known solution of velocity is divergence-free. The source term $\mathbf{f}$, initial and boundary conditions are chosen to correspond to the exact solution. The spatial discretization is effected via the 
Figure 1 A uniform mesh with $h=\sqrt{2} / 8$.

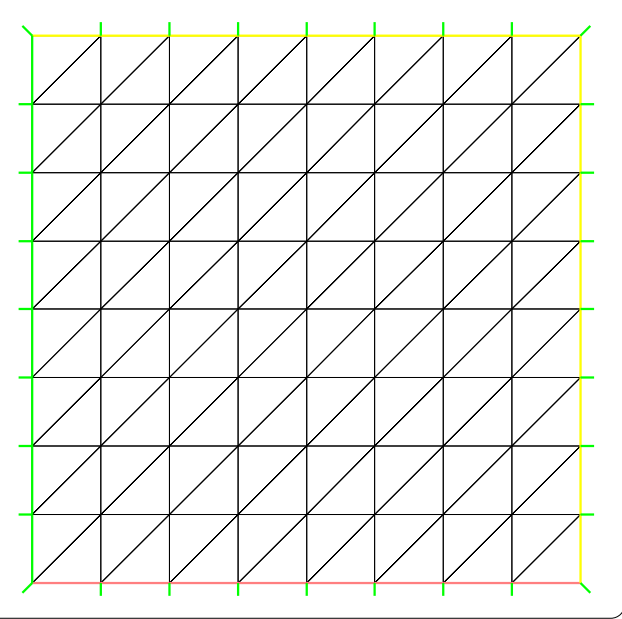

Table 1 Errors and CPU performance of the BDF2-LE scheme by using $\left(P_{1} b, P_{1}, P_{1} d c\right)$ finite element for $T=0.1$ and $\Delta t=0.1 h^{2}$

\begin{tabular}{clllllr}
\hline $\mathbf{1 / h}$ & $\left\|\boldsymbol{e}_{\boldsymbol{\sigma}}\right\|_{\boldsymbol{\infty}, \mathbf{0}}$ & $\left\|\boldsymbol{e}_{\boldsymbol{\sigma}}\right\|_{\mathbf{0}, \mathbf{0}}$ & $\left\|\boldsymbol{e}_{\mathbf{u}}\right\|_{\boldsymbol{\infty}, \mathbf{0}}$ & $\left\|\boldsymbol{\nabla} \boldsymbol{e}_{\boldsymbol{u}}\right\|_{\mathbf{0}, \mathbf{0}}$ & $\boldsymbol{\| \boldsymbol { e } _ { \boldsymbol { p } } \| _ { \mathbf { 0 } , \mathbf { 0 } }}$ & \multicolumn{1}{c}{$\boldsymbol{C P U}$} \\
\hline 4 & 0.0668476 & 0.020227 & 0.0144429 & 0.0680567 & 0.0802753 & 3.932 \\
6 & 0.0329998 & 0.0102078 & 0.00709982 & 0.0423537 & 0.0363262 & 16.068 \\
8 & 0.0197219 & 0.00603989 & 0.00417817 & 0.0309046 & 0.0204888 & 47.252 \\
12 & 0.00961065 & 0.00283642 & 0.00192262 & 0.0201723 & 0.00904258 & 223.86 \\
16 & 0.0058987 & 0.00166652 & 0.00109412 & 0.0150118 & 0.00501535 & 698.801 \\
24 & 0.00311194 & 0.000809214 & 0.000489492 & 0.00995059 & 0.00214579 & $3,569.78$ \\
32 & 0.00205163 & 0.000499604 & 0.000275582 & 0.00744755 & 0.00116269 & $11,565.2$ \\
order & 1.67091 & 1.78025 & 1.90816 & 1.06096 & 2.03938 & \\
\hline
\end{tabular}

Table 2 Errors and CPU performance of the BDF2-LE scheme by using $\left(P_{2}, P_{1}, P_{2} d c\right)$ finite element for $T=0.1$ and $\Delta t=0.1 h^{2}$

\begin{tabular}{clllllr}
\hline $\mathbf{1 / h}$ & $\left\|\boldsymbol{e}_{\boldsymbol{\sigma}}\right\|_{\boldsymbol{\infty}, \mathbf{0}}$ & $\left\|\boldsymbol{e}_{\boldsymbol{\sigma}}\right\|_{\mathbf{0}, \mathbf{0}}$ & $\left\|\boldsymbol{e}_{\mathbf{u}}\right\|_{\boldsymbol{\infty}, \mathbf{0}}$ & $\left\|\boldsymbol{\|} \boldsymbol{e}_{\boldsymbol{u}}\right\|_{\mathbf{0}, \mathbf{0}}$ & $\left\|\boldsymbol{e}_{\boldsymbol{p}}\right\|_{\mathbf{0}, \mathbf{0}}$ & \multicolumn{1}{c}{$\mathrm{CPU}$} \\
\hline 4 & 0.0092267 & 0.00275575 & 0.00172818 & 0.0144507 & 0.0803158 & 9.064 \\
6 & 0.00315424 & 0.000907616 & 0.00050577 & 0.0069492 & 0.0362654 & 36.925 \\
8 & 0.00151397 & 0.000411656 & 0.000206825 & 0.00401875 & 0.0204507 & 109.762 \\
12 & 0.000570131 & 0.000139673 & $5.93583 \mathrm{e}-005$ & 0.00182162 & 0.00903151 & 533.443 \\
16 & 0.000296275 & $6.74014 \mathrm{e}-005$ & $2.59447 \mathrm{e}-005$ & 0.00103212 & 0.00501414 & $1,732.81$ \\
24 & 0.000122468 & $2.56494 \mathrm{e}-005$ & $1.1174 \mathrm{e}-005$ & 0.000462319 & 0.00214968 & $8,761.63$ \\
32 & $6.67788 \mathrm{e}-005$ & $1.34476 \mathrm{e}-005$ & $8.7483 \mathrm{e}-006$ & 0.000262501 & 0.00116617 & $29,263.6$ \\
order & 2.3616 & 2.55223 & 2.50376 & 1.93062 & 2.03803 & \\
\hline
\end{tabular}

pairs $\left(P_{1} b, P_{1}, P_{1} d c\right)$ and $\left(P_{2}, P_{1}, P_{2} d c\right)$ to approximate velocity, pressure and stress tensor on a uniform triangular grid (see Figure 1 for $h=\sqrt{2} / 8$ ), respectively.

Tables 1 and 2 are numerical results of the BDF2-LE scheme (3.1a)-(3.1c) by using $\left(P_{1} b, P_{1}, P_{1} d c\right)$ elements and $\left(P_{2}, P_{1}, P_{2} d c\right)$ elements, respectively. We see that $\left\|D\left(e_{\mathbf{u}}\right)\right\|_{0,0}$ error has optimal convergence rate; however, $\left\|e_{\sigma}\right\|_{\infty, 0},\left\|e_{\sigma}\right\|_{0,0}$ and $\left\|e_{\mathbf{u}}\right\|_{\infty, 0}$ errors are not optimal, while $\left\|e_{p}\right\|_{0,0}$ is super-convergence for $\left(P_{1} b, P_{1}, P_{1} d c\right)$ elements.

Choosing $\triangle t=0.05 h$ and using $\left(P_{2}, P_{1}, P_{2} d c\right)$ elements, we present the results in Table 3 to verify time convergence order. It is easy to see that the time convergence order is two.

In order to test the computational efficiency, we compared the CPU time of the BDF2-LE scheme (Scheme 3.1) with the classical fully implicit BDF2 scheme (Scheme 5.1). 
Table 3 Errors and CPU performance of the BDF2-LE scheme by using $\left(P_{2}, P_{1}, P_{2} d c\right)$ finite element with $\Delta t=0.05 h$ and $T=0.1$

\begin{tabular}{llllllr}
\hline $\boldsymbol{\Delta} \boldsymbol{t}$ & $\left\|\boldsymbol{e}_{\boldsymbol{\sigma}}\right\|_{\boldsymbol{\infty}, \mathbf{0}}$ & $\left\|\boldsymbol{e}_{\boldsymbol{\sigma}}\right\|_{\mathbf{0}, \mathbf{0}}$ & $\left\|\boldsymbol{e}_{\mathbf{u}}\right\|_{\boldsymbol{\infty}, \mathbf{0}}$ & $\left\|\boldsymbol{\nabla} \boldsymbol{e}_{\boldsymbol{u}}\right\|_{\mathbf{0}, \mathbf{0}}$ & $\left\|\boldsymbol{e}_{\boldsymbol{p}}\right\|_{\mathbf{0}, \mathbf{0}}$ & \multicolumn{1}{c}{$\mathrm{CPU}$} \\
\hline $1 / 120$ & $3.09191 \mathrm{e}-3$ & $8.76703 \mathrm{e}-4$ & $5.05062 \mathrm{e}-4$ & $6.74744 \mathrm{e}-3$ & 0.0352161 & 16.61 \\
$1 / 160$ & $1.47401 \mathrm{e}-3$ & $3.98486 \mathrm{e}-4$ & $2.06665 \mathrm{e}-4$ & $3.92193 \mathrm{e}-3$ & 0.0199588 & 37.535 \\
$1 / 240$ & $5.53103 \mathrm{e}-4$ & $1.35618 \mathrm{e}-4$ & $5.93415 \mathrm{e}-5$ & $1.78953 \mathrm{e}-3$ & $8.87254 \mathrm{e}-3$ & 125.627 \\
$1 / 320$ & $2.87945 \mathrm{e}-4$ & $6.56174 \mathrm{e}-5$ & $2.58288 \mathrm{e}-5$ & $1.01788 \mathrm{e}-3$ & $4.94498 \mathrm{e}-3$ & 317.612 \\
$1 / 480$ & $1.19708 \mathrm{e}-4$ & $2.50901 \mathrm{e}-5$ & $1.11776 \mathrm{e}-5$ & $4.57881 \mathrm{e}-4$ & $2.12907 \mathrm{e}-3$ & $1,124.82$ \\
order & 2.35658 & 2.57344 & 2.78518 & 1.93818 & 2.02091 & \\
\hline
\end{tabular}

Table 4 Errors and CPU performance of the BDF2-nonlinear scheme by using $\left(P_{1} b, P_{1}, P_{1} d c\right)$ finite element for $T=0.1$ and $\Delta t=0.1 h^{2}$

\begin{tabular}{|c|c|c|c|c|c|c|}
\hline $1 / h$ & $\left\|\boldsymbol{e}_{\sigma}\right\|_{\infty, 0}$ & \|\|$e_{\sigma}\|\|_{0,0}$ & $\left\|e_{u}\right\|_{\infty, 0}$ & \|\|$\nabla \boldsymbol{e}_{u} \|_{0,0}$ & \|\|$e_{p} \|\left.\right|_{0,0}$ & CPU \\
\hline 4 & 0.0668514 & 0.0202271 & 0.0144429 & 0.0680566 & 0.0802749 & 12.651 \\
\hline 6 & 0.0330003 & 0.0102078 & 0.00709982 & 0.0423537 & 0.0363262 & 55.302 \\
\hline 8 & 0.0197219 & 0.00603989 & 0.00417817 & 0.0309046 & 0.0204888 & 113.306 \\
\hline 12 & 0.00961065 & 0.00283642 & 0.00192262 & 0.0201723 & 0.00904258 & 557.295 \\
\hline 16 & 0.0058987 & 0.00166652 & 0.00109412 & 0.0150118 & 0.00501535 & $1,759.45$ \\
\hline 24 & 0.00311194 & 0.000809214 & 0.000489492 & 0.00995059 & 0.00214579 & $9,120.06$ \\
\hline 32 & 0.00205163 & 0.000499604 & 0.000275582 & 0.00744755 & 0.00116269 & $29,252.6$ \\
\hline order & 1.67093 & 1.78026 & 1.90816 & 1.06096 & 2.03938 & \\
\hline
\end{tabular}

Scheme 5.1 (BDF2 fully implicit scheme) Given $\mathbf{u}_{h}^{-1}=\mathbf{u}_{h}^{0} \in V_{h}, \sigma_{h}^{-1}=\sigma_{h}^{0} \in S_{h}$, find $\left(\mathbf{u}_{h}^{n+1}, p_{h}^{n+1}, \sigma_{h}^{n+1}\right) \in X_{h} \times Q_{h} \times S_{h}$ for $n=0,1,2, \ldots, N-1$ such that

$$
\begin{aligned}
& \operatorname{Re}\left(\beth\left(\mathbf{u}_{h}^{n+1}\right), \mathbf{v}_{h}\right)+\operatorname{Rec}\left(\mathbf{u}_{h}^{n+1}, \mathbf{u}_{h}^{n+1}, \mathbf{v}_{h}\right)+\left(\sigma_{h}^{n+1}, D\left(\mathbf{v}_{h}\right)\right) \\
& \quad+2(1-\alpha)\left(D\left(\mathbf{u}_{h}^{n+1}\right), D\left(\mathbf{v}_{h}\right)\right)-\left(p_{h}^{n+1}, \nabla \cdot \mathbf{v}_{h}\right)=\left(\mathbf{f}^{n+1}, \mathbf{v}_{h}\right), \\
& \left(q_{h}, \nabla \cdot \mathbf{u}_{h}^{n+1}\right)=0, \\
& \lambda\left(\beth\left(\sigma_{h}^{n+1}\right), \tau_{h}\right)+\left(\sigma_{h}^{n+1}, \tau_{h}\right)+\lambda B\left(\mathbf{u}_{h}^{n+1}, \sigma_{h}^{n+1}, \tau_{h}\right) \\
& \quad-2 \alpha\left(D\left(\mathbf{u}_{h}^{n+1}\right), \tau_{h}\right)+\lambda\left(g_{a}\left(\sigma_{h}^{n+1}, \nabla \mathbf{u}_{h}^{n+1}\right), \tau_{h}\right)=0
\end{aligned}
$$

for all $\left(\mathbf{v}_{h}, q_{h}, \tau_{h}\right) \in X_{h} \times Q_{h} \times S_{h}$.

Unlike the BDF2-LE, the classical fully implicit BDF2 presented in Scheme 5.1 requires to solve a nonlinear problem at each time level. We employ the Newton iterative method. When relative nonlinear residual is less than $10^{-8}$, the Newton iteration is stopped. The results of Scheme 5.1 are presented in Tables 4 and 5.

Comparing Tables 1-2 with Tables 4-5, respectively, we find that two numerical schemes have the same level of accuracy, while the BDF2-LE scheme can save significant CPU time for both $\left(P_{1} b, P_{1}, P_{1} d c\right)$ elements and $\left(P_{2}, P_{1}, P_{2} d c\right)$ elements.

\subsection{4-to-1 planar contraction flow}

Numerical simulations of viscoelastic flow through a planar or axisymmetric contraction have been widely studied in [42, 43]. Here the case of planar flow through a contraction geometry with a ratio of 4:1 with respect to upstream and downstream channel widths is considered. The contraction angle is fixed $3 \pi / 2$, and the channel lengths are sufficiently long to impose a fully developed Poiseuille flow in the inflow and outflow channels. The 
Table 5 Errors and CPU performance of a fully implicit BDF2 scheme by using $\left(P_{2}, P_{1}, P_{2} d c\right)$ finite element for $T=0.1$, and $\Delta t=0.1 h^{2}$

\begin{tabular}{clllllr}
\hline $\mathbf{1 / h}$ & $\left\|\boldsymbol{e}_{\boldsymbol{\sigma}}\right\|_{\boldsymbol{\infty}, \mathbf{0}}$ & $\left\|\boldsymbol{e}_{\boldsymbol{\sigma}}\right\|_{\mathbf{0}, \mathbf{0}}$ & $\left\|\boldsymbol{e}_{\mathbf{u}}\right\|_{\boldsymbol{\infty}, \mathbf{0}}$ & $\left\|\boldsymbol{\|} \boldsymbol{e}_{\boldsymbol{u}}\right\|_{\mathbf{0}, \mathbf{0}}$ & $\left\|\boldsymbol{e}_{\boldsymbol{p}}\right\|_{\mathbf{0}, \mathbf{0}}$ & \multicolumn{1}{c}{$\mathrm{CPU}$} \\
\hline 4 & 0.00922671 & 0.00275576 & 0.00172819 & 0.0144507 & 0.0803158 & 29.361 \\
6 & 0.00315424 & 0.000907616 & 0.00050577 & 0.0069492 & 0.0362654 & 90.013 \\
8 & 0.00151397 & 0.000411656 & 0.000206825 & 0.00401875 & 0.0204507 & 279.113 \\
12 & 0.000570131 & 0.000139673 & $5.93583 \mathrm{e}-005$ & 0.00182162 & 0.00903151 & $1,416.41$ \\
16 & 0.000296275 & $6.74015 \mathrm{e}-005$ & $2.59467 \mathrm{e}-005$ & 0.00103212 & 0.00501414 & $4,432.56$ \\
24 & 0.000122468 & $2.56494 \mathrm{e}-005$ & $1.08 \mathrm{e}-005$ & 0.000462319 & 0.00214968 & $22,743.7$ \\
32 & $6.67788 \mathrm{e}-005$ & $1.34476 \mathrm{e}-005$ & $8.74507 \mathrm{e}-006$ & 0.000262501 & 0.00116617 & $75,954.1$ \\
order & 2.3616 & 2.55223 & 2.49823 & 1.93062 & 2.03803 & \\
\hline
\end{tabular}

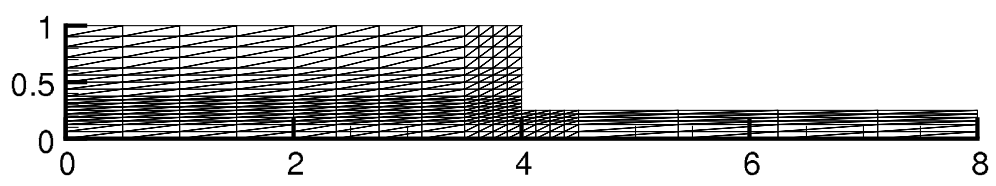

Figure 2 Plot of 4:1 contraction domain geometry and sample contraction mesh.

geometry of the computational domain is illustrated in Figure 2. The lower left corner of the domain corresponds to $x=y=0$.

The computations of the mesh are also shown in Figure 2 with $\Delta x_{\min }=0.0625$ and $\Delta y_{\min }=0.015625$. We denote $\Gamma_{\text {in }}=\{(x, y): x=0,0 \leq y \leq 1\}$ and $\Gamma_{\text {out }}=\{(x, y): x=8,0 \leq$ $y \leq 0.25\}$. On this domain the velocity boundary conditions are

$$
\mathbf{u}=\left[\begin{array}{c}
\frac{1}{32}\left(1-y^{2}\right) \\
0
\end{array}\right] \quad \text { on } \Gamma_{\text {in }}, \quad \mathbf{u}=\left[\begin{array}{c}
2\left(\frac{1}{16}-y^{2}\right) \\
0
\end{array}\right] \text { on } \Gamma_{\text {out }} .
$$

For stress tensor $\sigma$ on $\Gamma_{\text {in }}$,

$$
\begin{aligned}
& \sigma_{11}=\frac{-\alpha \lambda(a+1)(-y / 16)^{2}}{\left(a^{2}-1\right) \lambda^{2}(-y / 16)^{2}-1}, \\
& \sigma_{12}=\sigma_{21}=\frac{-\alpha(-y / 16)}{\left(a^{2}-1\right) \lambda^{2}(-y / 16)^{2}-1}, \\
& \sigma_{22}=\frac{-\alpha \lambda(a-1)(-y / 16)^{2}}{\left(a^{2}-1\right) \lambda^{2}(-y / 16)^{2}-1} .
\end{aligned}
$$

Symmetry conditions are imposed on the bottom of the computational domain. Besides, the parameters $\operatorname{Re}, \alpha, \lambda$ and $a$ are chosen to be $1,8 / 9,0.7$ and 1 , respectively.

We performed the following study: starting from rest, we measured the time that the approximation solution reaches a steady state by using $\left(P_{1} b, P_{1}, P_{1} d c\right)$ elements. The criterion to stop this process is the following:

$$
\max \left\{\frac{\left\|\mathbf{u}_{h}^{n+1}-\mathbf{u}_{h}^{n}\right\|}{\left\|\mathbf{u}_{h}^{n+1}\right\|}, \frac{\left\|\sigma_{h}^{n+1}-\sigma_{h}^{n}\right\|}{\left\|\sigma_{h}^{n+1}\right\|}\right\} \leq 10^{-5},
$$

where $n+1, n$ denote $t_{n+1}, t_{n}$, respectively.

We plot the evolution of the kinetic energy $0.5 *\left\|\mathbf{u}_{h}^{n+1}\right\|^{2}$ and $0.5 *\left\|\sigma_{h}^{n+1}\right\|^{2}$ using time step $\Delta t=0.01$ until it reaches its steady state in Figure 3 and Figure 4, respectively. We 
Figure 3 Evolution of $0.5 *\left\|u_{h}^{n+1}\right\|^{2}$ in time with time step $\Delta t=0.01$ by using $\left(P_{1} b, P_{1}, P_{1} d c\right)$ element.

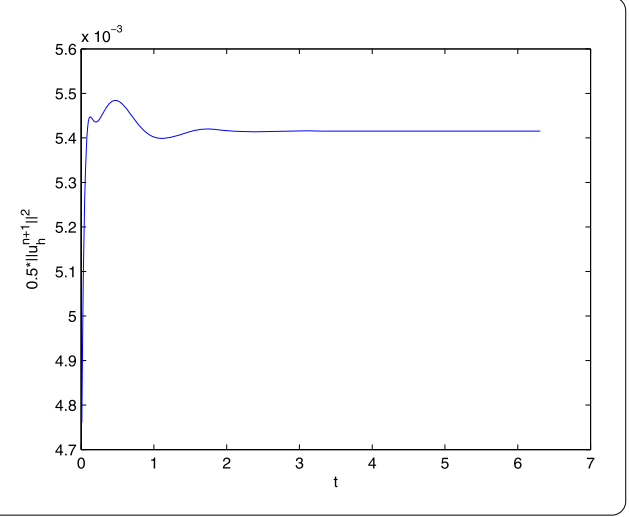

Figure 4 Evolution of $0.5 *\left\|\sigma_{h}^{n+1}\right\|^{2}$ in time with time step $\Delta t=0.01$ by using $\left(P_{1} b, P_{1}, P_{1} d c\right)$ element.

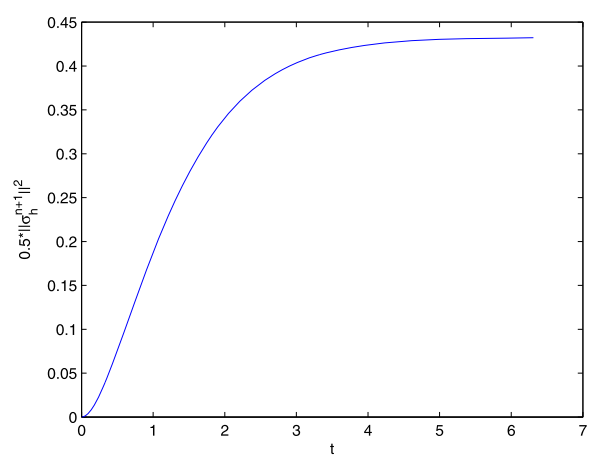

Figure 5 Horizontal velocity $u_{1}$ near reentrant corner $(x=4.0625)$. The mark ' $O$ ' indicates results for the steady problem and ' $t$ ' indicates results for the time-dependent problem at final time $t=6.31$ with time step $\Delta t=0.01$ and $\left(P_{1} b, P_{1}, P_{1} d c\right)$ element.

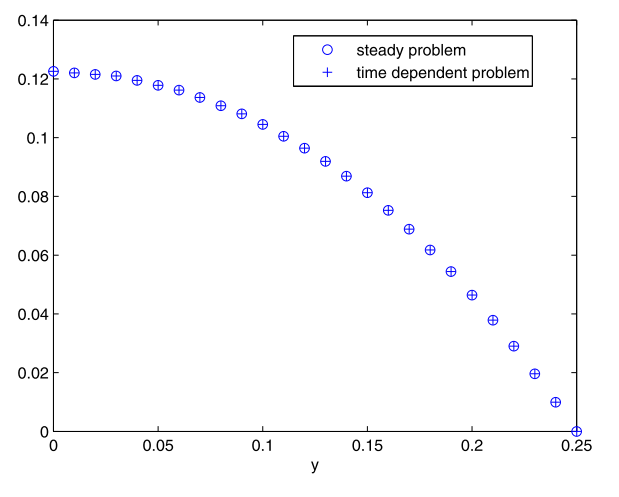

observe that it converges towards a steady state, while the kinetic energy of velocity has some oscillations at the beginning.

Figures 5 and 6 present the horizontal and vertical velocities near the reentrance corner along the vertical line $x=4.0625$. We observe that the horizontal velocity is almost continuous, while the vertical velocity has high gradients near $y=0.23$. However, we find that the solutions of the time-dependent problem can converge to the solutions of the steady problem.

We plot the streamlines of velocity for the steady problem and the time-dependent problem at final time $t=6.31$ in Figure 7 and Figure 8, respectively. It is easy to observe that the two figures are almost alike. 
Figure 6 Vertical velocity $u_{2}$ near reentrant corner $(x=4.0625)$. The mark ' $O$ ' indicates results for the steady problem and ' + ' indicates results for the time-dependent problem at final time $t=6.31$

with time step $\Delta t=0.01$ and $\left(P_{1} b, P_{1}, P_{1} d c\right)$ element.

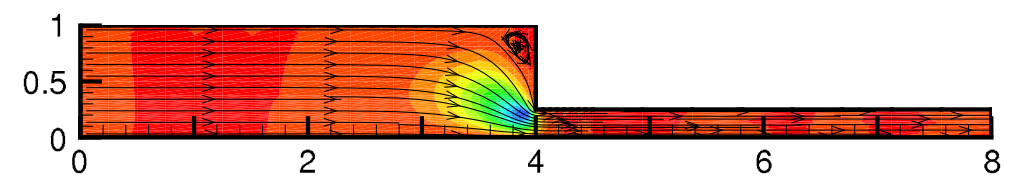

Figure 7 Streamlines of velocity contours for u: steady problem.

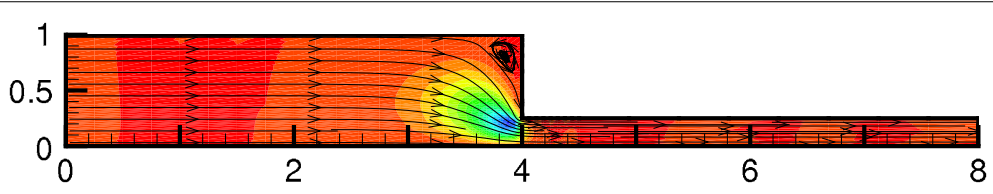

Figure 8 Streamlines of velocity contours for u: time-dependent problem at final time $t=6.31$.

\section{Conclusions and discussions}

In this work, we have applied the BDF2-LE time-stepping scheme with Galerkin finite element to solve the time-dependent viscoelastic fluid flow in $\mathbb{R}^{d}, d=2,3$. We establish the stability analysis and a priori error estimates. Some numerical tests are provided to support the theoretical results and to demonstrate the effectiveness of the method.

Also, our analysis can be easily extended to the BDF2-LE decoupled scheme and other nonlinear viscoelastic fluid flow.

Acknowledgements

We are thankful to the editor and the anonymous reviewers for many valuable suggestions to improve this paper.

Funding

The first author is partially supported by the National Natural Science Foundation of China (11401174), the Educational Commission of Henan Province of China (14B110021) and the Henan Scientific and Technological Research Project (132102310309)

\section{Competing interests}

The authors declare that they have no competing interests.

Authors' contributions

The study was carried out in collaboration among all authors. YZZ carried out the main theorem and wrote the paper; CX revised and checked the paper; and JQZ checked the article. All authors read and approved the final manuscript.

\section{Author details}

${ }^{1}$ School of Mathematics and Statistics, Henan University of Science and Technology, Luoyang, 471023, China. ${ }^{2}$ Faculty of Mathematics and Physics Education, Luoyang Institute of Science and Technology, Luoyang, 471023, China. 


\section{Publisher's Note}

Springer Nature remains neutral with regard to jurisdictional claims in published maps and institutional affiliations.

Received: 18 May 2017 Accepted: 12 September 2017 Published online: 25 September 2017

\section{References}

1. Oldroyd, JG: On the formulation of rheological equations of state. Proc. R. Soc. Lond. A 200, 523-541 (1950)

2. White, JL, Metzner, AB: Development of constitutive equations for polymeric melts and solutions. J. Appl. Polym. Sci. 7, 1867-1889 (1963)

3. Johnson, MW, Segalman, D: A model for viscoelastic fluid behavior which allows non-affine deformation. J. Non-Newton. Fluid Mech. 2, 255-270 (1977)

4. Renardy, M: Mathematical Analysis of Viscoelastic Flows, vol. 65. SIAM, Philadelphia (2000)

5. Guillope, C, Saut, JC: Existence results for the flow of viscoelastic fluids with a differential constitutive law. Nonlinear Anal. 15, 849-869 (1990)

6. Baranger, J, Sandri, D: Finite element approximation of viscoelastic fluid flow: existence of approximate solutions and error bounds, I. Discontinuous constraints. Numer. Math. 63, 13-27 (1992)

7. Sandri, D: Finite element approximation of viscoelastic fluid flow: existence of approximate solutions and error bounds, continuous approximation of the stress. SIAM J. Numer. Anal. 31, 362-377 (1994)

8. Lee, H: A multigrid method for viscoelastic fluid flow. SIAM J. Numer. Anal. 42, 109-129 (2004)

9. Howell, JS: Computation of viscoelastic fluid flows using continuation methods. J. Comput. Appl. Math. 225, 187-201 (2009)

10. Zhang, YZ, Hou, YR, Mu, BY: Defect correction method for time-dependent viscoelastic fluid flow. Int. J. Comput. Math. 88, 1546-1563 (2011)

11. Baranger, J, Wardi, S: Numerical analysis of an FEM for a transient viscoelastic flow. Comput. Methods Appl. Mech. Eng. 125, 171-185 (1995)

12. Ervin, VJ, Miles, WW: Approximation of time-dependent, viscoelastic fluid flow: SUPG approximation. SIAM J. Numer. Anal. 41, 457-486 (2003)

13. Ervin, VJ, Miles, WW: Approximation of time-dependent, multi-component, viscoelastic fluid flow. Comput. Methods Appl. Mech. Eng. 194, 2229-2255 (2005)

14. Ervin, VJ, Heuer, N: Approximation of time-dependent, viscoelastic fluid flow: Crank-Nicolson, finite element approximation. Numer. Methods Partial Differ. Equ. 20, 248-283 (2004)

15. Bensaada, M, Esselaoui, D: Error estimates for a stabilized finite element method for the Oldroyd B model. J. Math. Anal. Appl. 325, 1042-1059 (2007)

16. Chrispell, JC, Ervin, VJ, Jenkins, EW: A fractional step $\theta$-method approximation of time dependent viscoelastic fluid flow. J. Comput. Appl. Math. 232, 159-175 (2009)

17. Bonito, A, Clément, $\mathrm{P}$, Picasso, M: Mathematical and numerical analysis of a simplified time dependent viscoelastic flow. Numer. Math. 107, 213-255 (2007)

18. Zheng, HB, Yu, JP, Shan, L: Unconditional error estimates for time dependent viscoelastic fluid flow. Appl. Numer. Math. 119, 1-17 (2017)

19. Hairer, E, Wanner, G: Solving Ordinary Differential Equations II: Stiff and Differential-Algebraic Problems, 2nd edn. Springer, Berlin (1996)

20. Baker, GA, Dougalis, VA, Karakashian, OA: On a higher order accurate fully discrete Galerkin approximation to the Navier-Stokes equations. Math. Comput. 39, 339-375 (1982)

21. Girault, V, Raviart, PA: Finite Element Method for Navier-Stokes Equations. Springer, Berlin (1986)

22. Hundsdorfer, W: Partially implicit BDF2 blends for convection dominated flows. SIAM J. Numer. Anal. 38, 1763-1783 (2001)

23. Varah, JM: Stability restrictions on second order, three level finite difference schemes for parabolic equations. SIAM J. Numer. Anal. 17, 300-309 (1980)

24. Ravindran, SS: Convergence of extrapolated BDF2 finite element schemes for unsteady penetrative convection model. Numer. Funct. Anal. Optim. 33, 48-79 (2012)

25. Ravindran, SS: A second-order backward difference time-stepping scheme for penalized Navier-Stokes equations modeling filtration through porous media. Numer. Methods Partial Differ. Equ. 32, 681-705 (2016)

26. Ravindran, SS: An extrapolated second order backward difference time-stepping scheme for the magnetohydrodynamics system. Numer. Funct. Anal. Optim. 37, 990-1020 (2016)

27. Layton, W, Tran, $\mathrm{H}$, Trenchea, C: Numerical analysis of two partitioned methods for uncoupling evolutionary MHD flows. Numer. Methods Partial Differ. Equ. 30, 1083-1102 (2014)

28. Akbas, M, Kaya, S, Rebholz, LG: On the stability at all times of linearly extrapolated BDF2 timestepping for multiphysics incompressible flow problems. Numer. Methods Partial Differ. Equ. 33, 999-1017 (2017)

29. Adams, RA: Sobolev Space. Pure and Applied Mathematics, vol. 65. Academic Press, New York (1975)

30. Zheng, HB, Shan, L, Hou, YR: A quadratic equal-order stabilized method for Stokes problem based on two local Gauss integrations. Numer. Methods Partial Differ. Equ. 26, 1180-1190 (2010)

31. Hou, YR, Li, KT: A small eddy correction method for nonlinear dissipative evolutionary equations. SIAM J. Numer. Anal. 41, 1101-1130 (2003)

32. Liu, QF, Hou, YR: A postprocessing mixed finite element method for the Navier-Stokes equations. Int. J. Comput. Fluid Dyn. 23, 461-475 (2009)

33. Hou, YR, Li, KT: Postprocessing Fourier Galerkin method for the Navier-Stokes equations. SIAM J. Numer. Anal. 47, 1909-1922 (2009)

34. Heywood, JG, Rannacher, R: Finite-element approximations of the nonstationary Navier-Stokes problem, part I: regularity of solutions and second-order spatial discretization. SIAM J. Numer. Anal. 19, 275-311 (1982)

35. Heywood, JG, Rannacher, R: Finite-element approximation of the nonstationary Navier-Stokes problem, part IV: error analysis for second-order. SIAM J. Numer. Anal. 27, 353-384 (1990)

36. Galvin, KJ: New subgrid artificial viscosity Galerkin methods for the Navier-Stokes equations. Comput. Methods Appl. Mech. Eng. 200, 242-250 (2011) 
37. Shang, YQ: Error analysis of a fully discrete finite element variational multiscale method for time dependent incompressible Navier-Stokes equations. Numer. Methods Partial Differ. Equ. 29, 2025-2046 (2013)

38. Zhang, YZ, Hou, YR, Zhao, JP: Error analysis of a fully discrete finite element variational multiscale method for the natural convection problem. Comput. Math. Appl. 68, 543-567 (2014)

39. Ervin, VJ, Howell, JS, Lee, H: A two-parameter defect-correction method for computation of steady-state viscoelastic fluid flow. Appl. Math. Comput. 196, 818-834 (2008)

40. He, YN, Sun, WW: Stability and convergence of the Crank-Nicolson/Adams-Bashforth scheme for the time-dependent Navier-Stokes equations. SIAM J. Numer. Anal. 45, 837-869 (2007)

41. Hecht, F, Pironneau, O, Hyaric, AL, Ohtsuka, K: FreeFem++, http://www.freefem.org/ff++ (2011)

42. Baaijens, FPT: Mixed finite element methods for viscoelastic flow analysis: a review. J. Non-Newton. Fluid Mech. 79, 361-385 (1998)

43. Owens, RG, Phillips, TN: Computational Rheology. Imperial College Press, London (2002)

Submit your manuscript to a SpringerOpen ${ }^{\circ}$ journal and benefit from:

- Convenient online submission

Rigorous peer review

Open access: articles freely available online

- High visibility within the field

- Retaining the copyright to your article

Submit your next manuscript at $\boldsymbol{\nabla}$ springeropen.com 